\title{
2D ELECTROMAGNETIC ACTUATED QUASI-STATIC FPCB BASED MICROMIRROR FOR LASER MARKING/ ENGRAVING SYSTEM
}

\author{
By
}

Devanshu Kakkar

Bachelor of Technology, Mechanical Engineering, Uttar Pradesh Technical University, 2014

\author{
A thesis \\ presented to Ryerson University \\ in partial fulfillment of the \\ requirements for the degree of \\ Master of Applied Science \\ in the program of \\ Mechanical and Industrial Engineering \\ Toronto, Ontario, Canada, 2019
}

(C) Devanshu Kakkar, 2019 


\section{AUTHOR'S DECLARATION FOR ELECTRONIC SUBMISSION OF A THESIS}

I hereby declare that I am the sole author of this thesis. This is a true copy of the thesis, including any required final revisions, as accepted by my examiners.

I authorize Ryerson University to lend this thesis to other institutions or individuals for the purpose of scholarly research.

I further authorize Ryerson University to reproduce this thesis by photocopying or by other means, in total or in part, at the request of other institutions or individuals for scholarly research.

I understand my thesis may be made electronically available to the public. 


\begin{abstract}
2D Electromagnetic Actuated Quasi-Static FPCB Based Micromirror for Laser Marking/ Engraving System

Master of Applied Science, 2019

Devanshu Kakkar

Mechanical \& Industrial Engineering

Ryerson University
\end{abstract}

This thesis presents the design and development of quasi-static 2-dimensional Flexible Printed Circuit Board (FPCB) based micromirror for portable laser marking/ engraving system. It focuses on the modelling and prototyping of the novel 2-dimensional electromagnetic actuated micromirror which can be used in place of two 1-dimensional micromirrors having the benefits of lower footprint in size of the scanner and efficient alignment with the incident laser beam. This 2dimensional micromirror can be used in portable low power laser marking/ engraving system ideally designed for consumer applications like printing name cards, engraving jewellery etc. The problem of drifting/ creep associated with quasi-static mode of FPCB based micromirror actuators is analyzed and Zero method is proposed to minimize the drifting issue which is proven experimentally in the thesis. From this thesis work, it is evident that the developed 2-dimensional, low-cost, quasi-static FPCB based micromirror would be a suitable candidate for replacement of the expensive conventional MEMS bonded mirrors in the market. 


\section{Acknowledgement}

I would like to express my sincere gratitude to my supervisor Dr. Siyuan He, who gave me the golden opportunity to pursue my Master of Applied Science program. During this program, he always encouraged and motivated me to complete my research successfully.

I convey my thanks to Bella Yang (Fastline circuits), Mateusz Szymanski (OPT lasers technology) and CMC Microsystems for their technical help and support regarding the FPCB circuits and Lasers systems.

I thank my fellow lab mates Karlmarx, Josh and Mehraz, and Hui Zho. They gave me ample support to pace up my research work.

I also thank my friends Sunny, Tushar, Rishi, and Ashish for their valuable help. My special thanks to Gurdeep Singh Ahluwalia for his blessing and moral support to me.

Finally, I dedicate this thesis to my father (Sunil Kakkar), mother (Anita Kakkar), uncle (Ved Kaila), aunt (Madhu Kaila) and my sister (Neha \& her family), as this degree would not have been possible without their support and sacrifices. 


\section{Table of Contents}

Author's declaration for electronic submission of a thesis ..........................................................ii

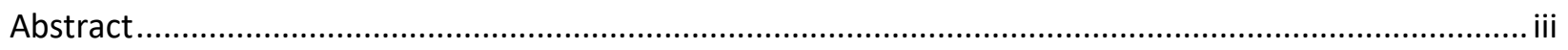

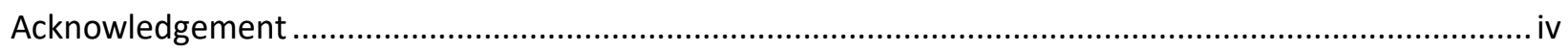

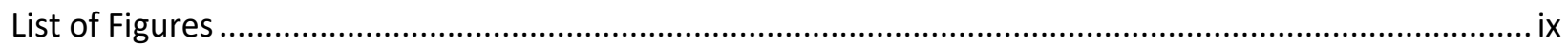

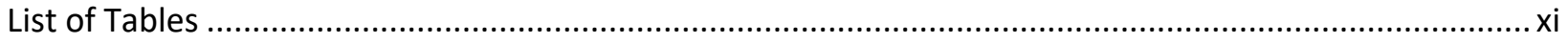

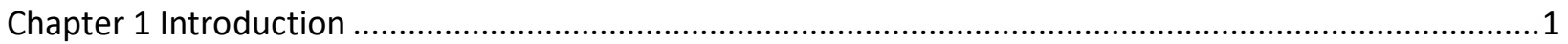

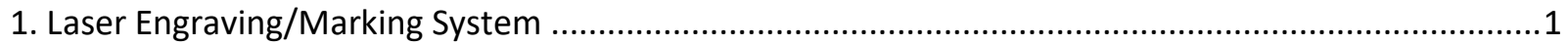

1.1 Difference between Laser Marking and Laser Engraving ............................................................1

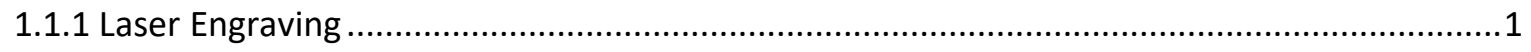

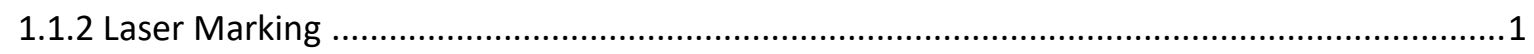

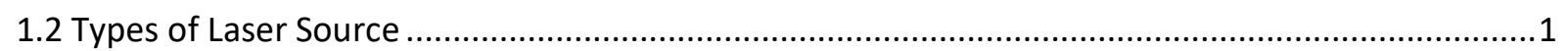

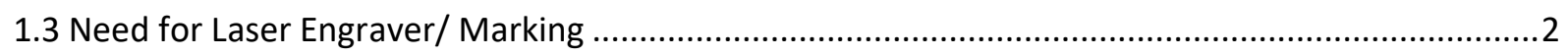

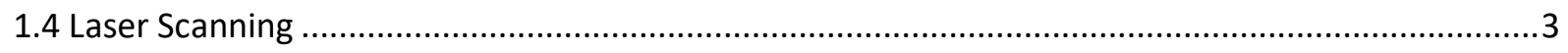

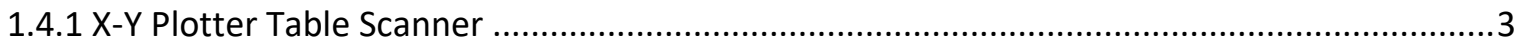

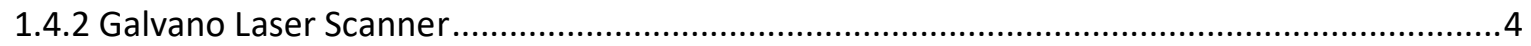

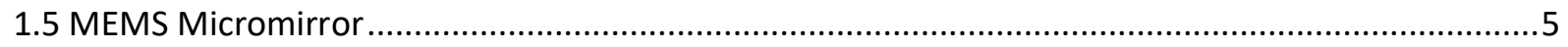

1.6 NEXGEN Laser Marker by Worldstar Technologies ......................................................................

1.7 NLL10 Label Laser by Nanosec Technology Gmbh .................................................................6

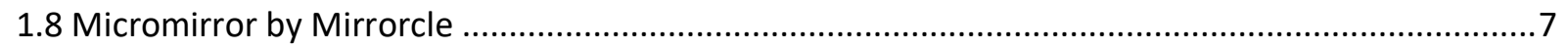

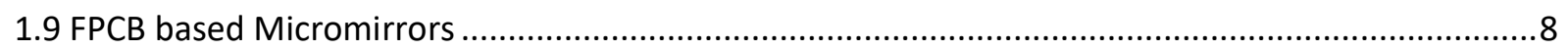

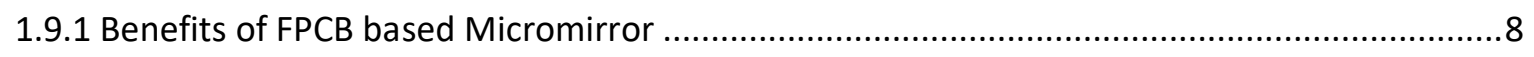

1.9.2 FPCB Based Scanners for Laser Marking/ Engraving ..................................................

1.9.3 Limitations of the Configuration of two 1-Dimensional FPCB Scanners ..............................

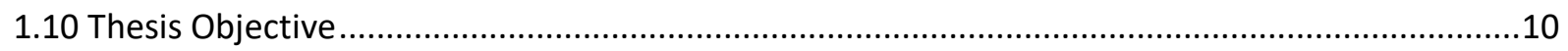

Chapter 2 Design of FPCB Micromirror and the Engraving/ Marking System .........................................12

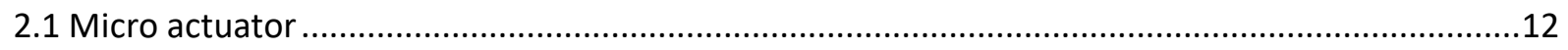

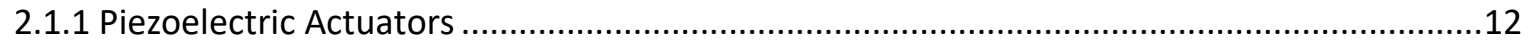

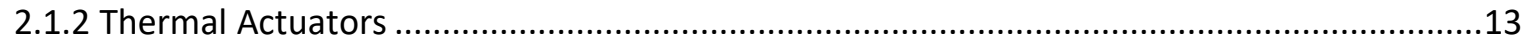

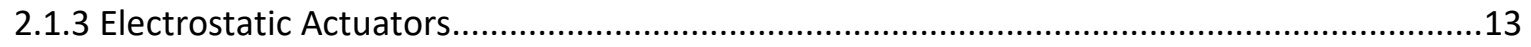




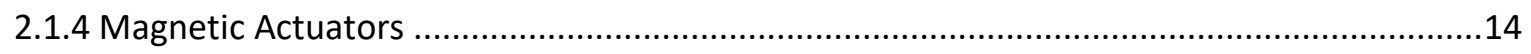

2.2 Design of Electromagnetic based FPCB Micromirror ...............................................................16

2.2.1 Working of the Electromagnetic Attraction/ Repulsion based FPCB actuator ....................16

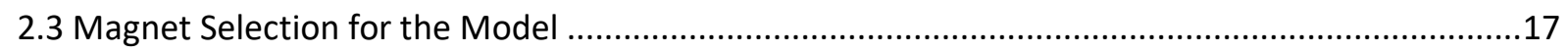

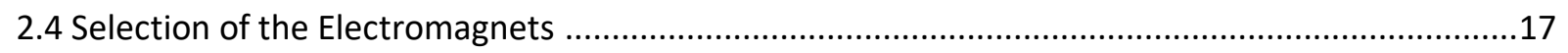

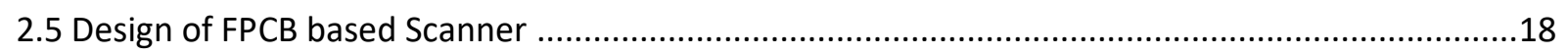

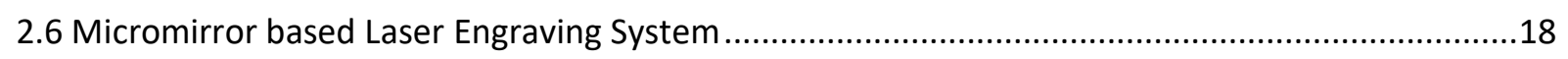

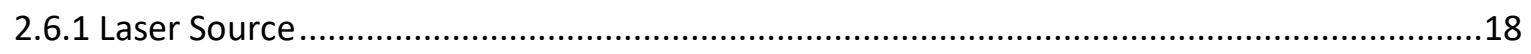

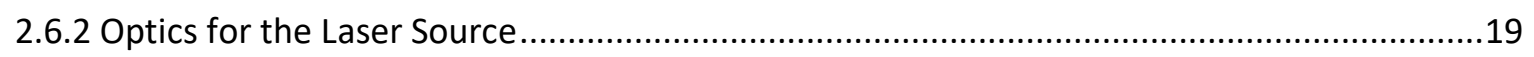

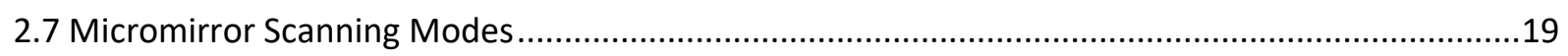

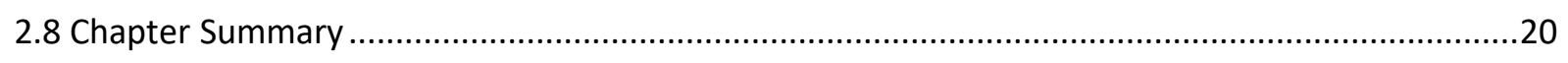

Chapter 3 Modelling and Simulation of the FPCB Micromirror based Engraving System .......................21

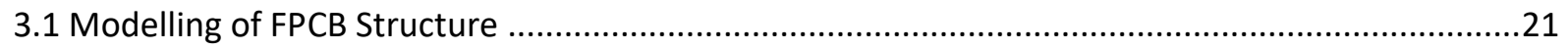

3.2 Modelling of the Mechanical Support Structure for the Micromirror .........................................25

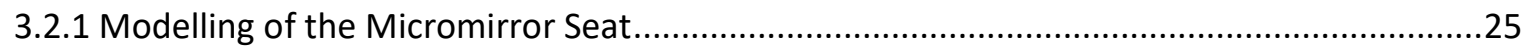

3.2.2 Modelling of the Electromagnet Support Structure ......................................................27

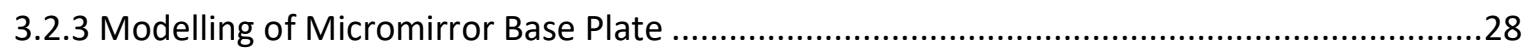

3.2.4 The Assembly of the Laser Marking/ Engraving Micromirror Scanner ...............................29

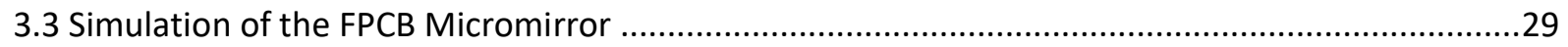

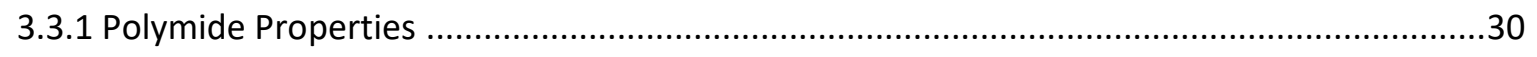

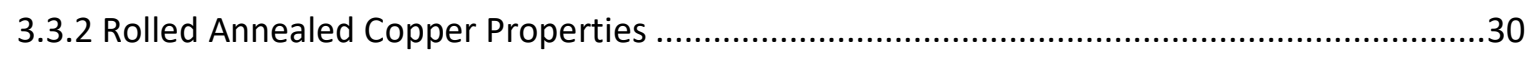

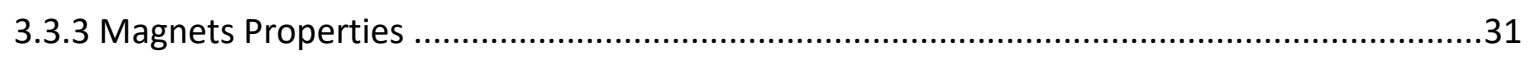

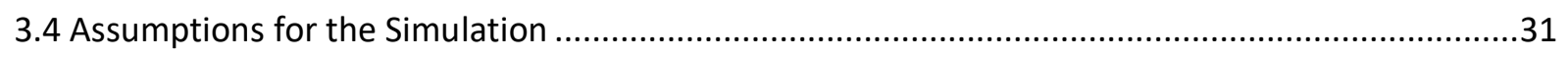

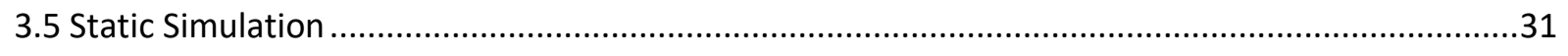

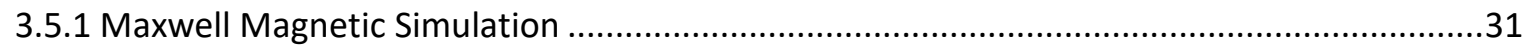

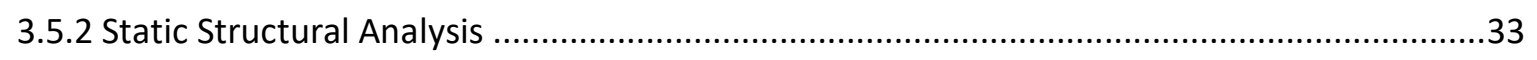

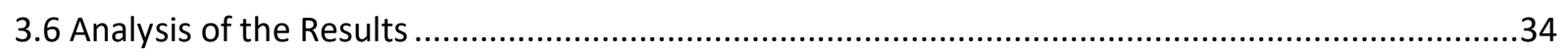

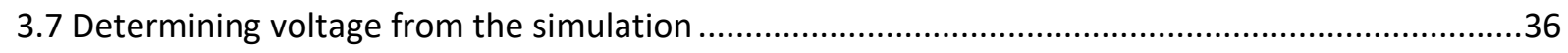

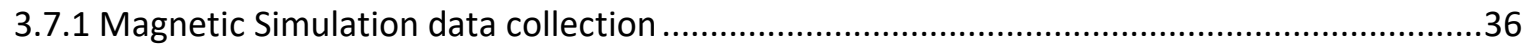


3.7.2 Static simulation

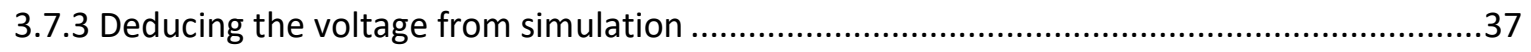

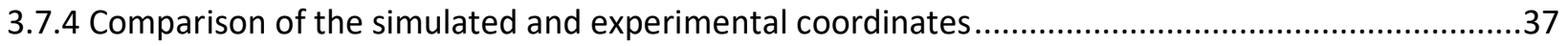

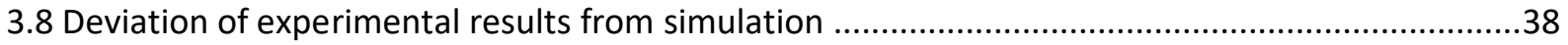

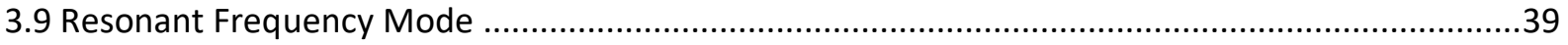

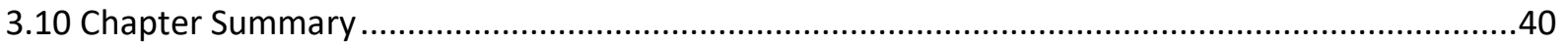

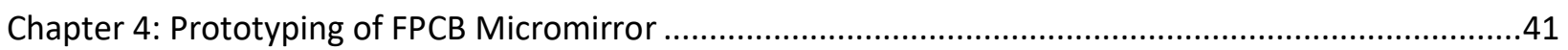

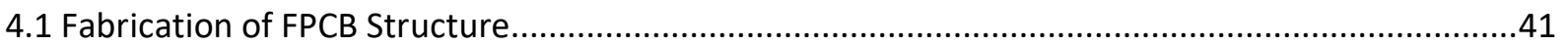

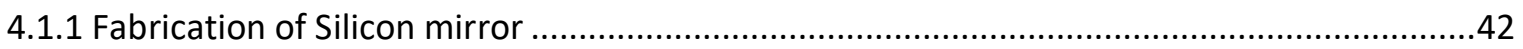

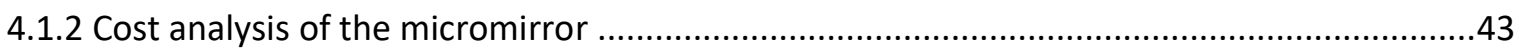

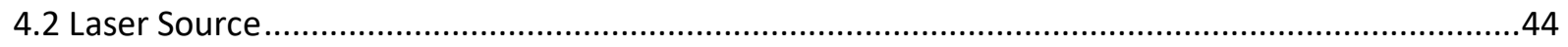

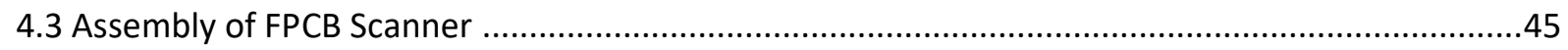

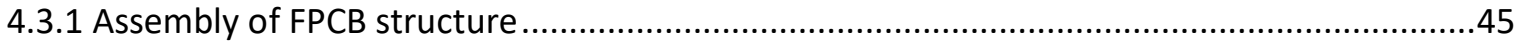

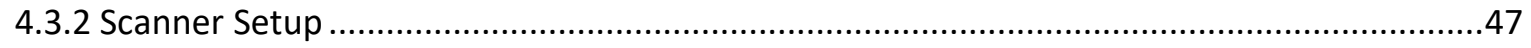

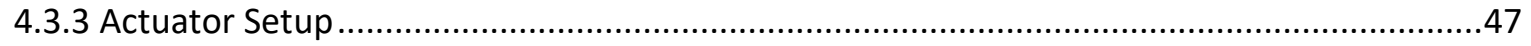

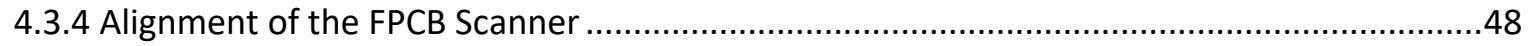

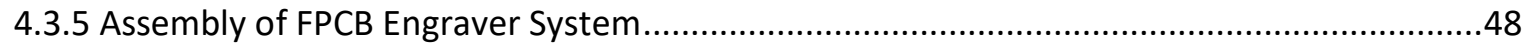

4.4 Controlling of the Micromirror: LabView Code …........................................................................

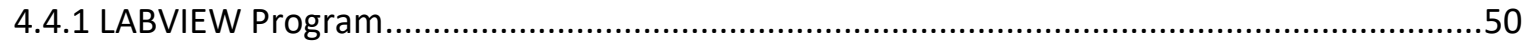

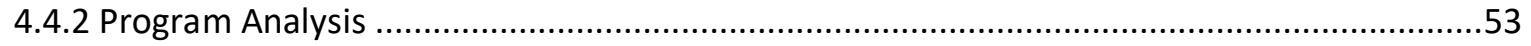

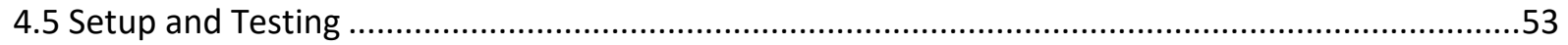

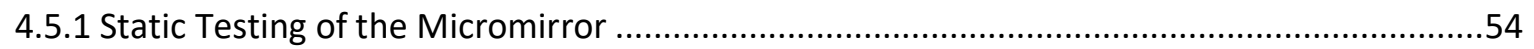

4.5.2 Resonant Frequency Test for the Micromirror.........................................................56

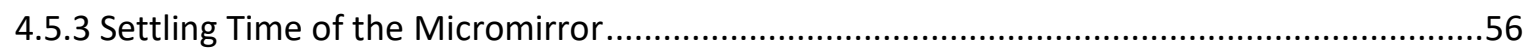

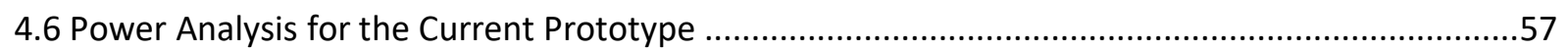

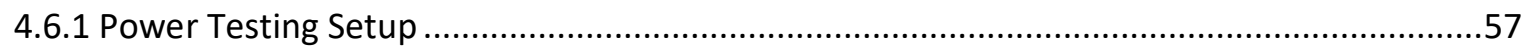

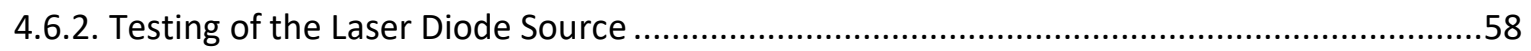

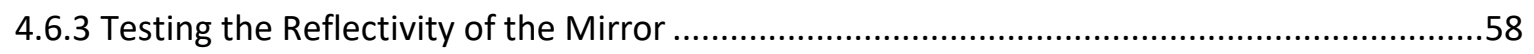




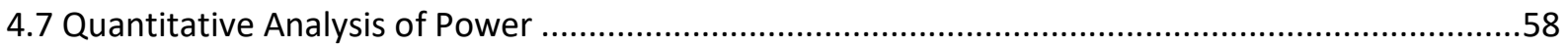

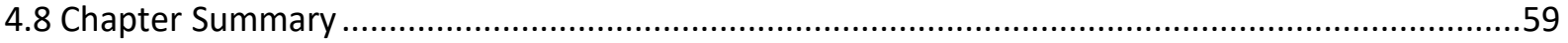

Chapter 5 Control Method for Laser Marking/ Engraving System and Method of overcoming Drifting....60

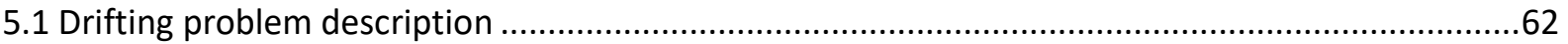

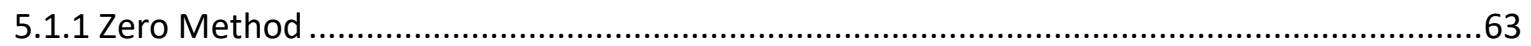

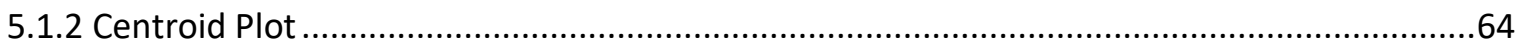

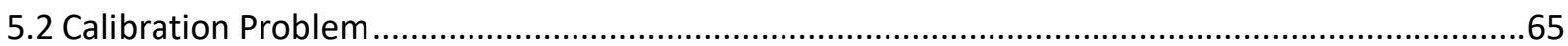

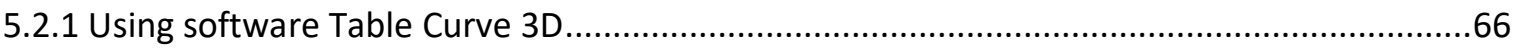

5.3 Experimental Verification and Steps for Engraving Pattern...................................................67

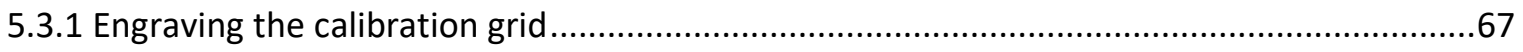

5.3.2 Plotting the Points into Software Table Curve 3D .........................................................6

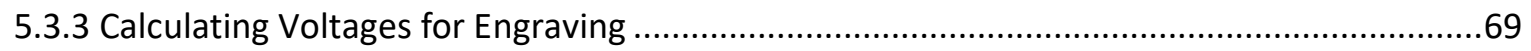

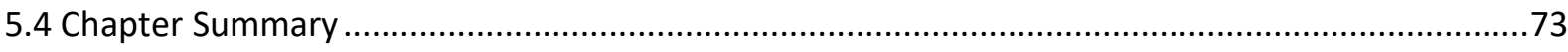

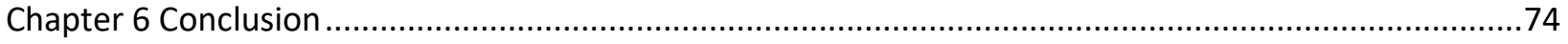

Appendix

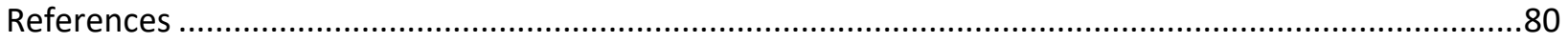




\section{List of Figures}

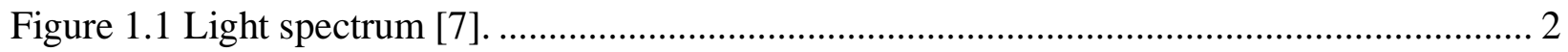

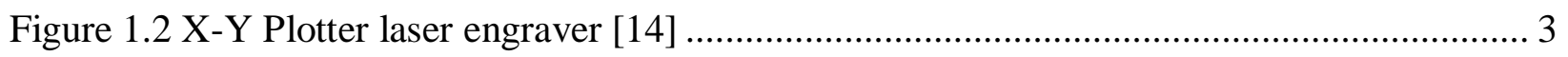

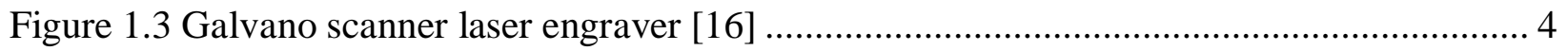

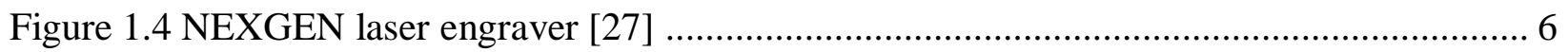

Figure 1.5 Laser engraver NLL 10 [29] ...................................................................... 7

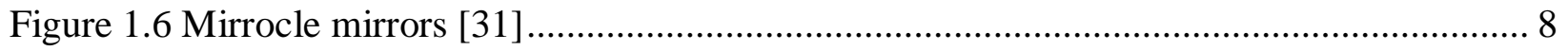

Figure 2.1 Schematic diagram of MEMS piezoelectric bending/ twisting micromirror [35]....... 13

Figure 2.2 Gimbal based micromirror ........................................................................ 14

Figure 2.3 Unidirectional FPCB based micromirror [32] .............................................. 15

Figure 2.4 Attraction/ repulsion of magnet and electromagnet .......................................... 15

Figure 3.1 Top and Isometric view of FPCB micromirror .............................................. 23

Figure 3.2 Control points of the spline of the FPCB micromirror .......................................... 25

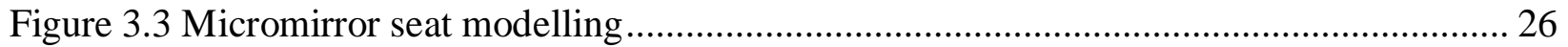

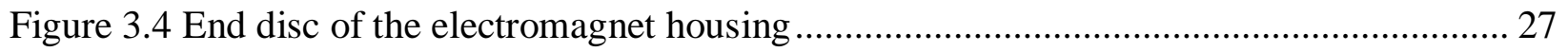

Figure 3.5 Front-end for the electromagnet micromirror scanner ......................................... 28

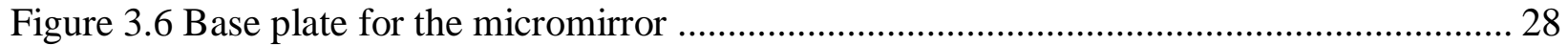

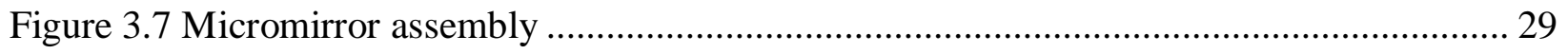

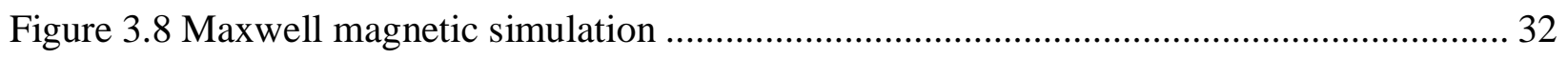

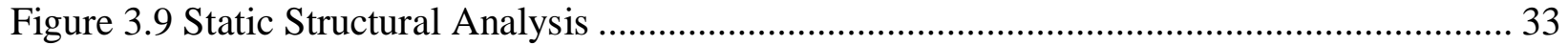

Figure 3.10 Marking the laser line point on the $200 \mathrm{~mm}$ far plane for coordinates calculation .... 34

Figure 3.11 Repulsive and Attractive Force between magnet and the electromagnet ................. 36

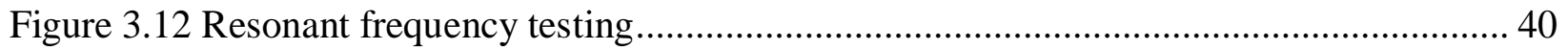

Figure 4.1 Different design of FPCB substrate ........................................................... 41

Figure 4.2 Reflectance at different wavelength [49] ........................................................ 43

Figure 4.3 OPT Laser module with pre-installed focusing lens ......................................... 45

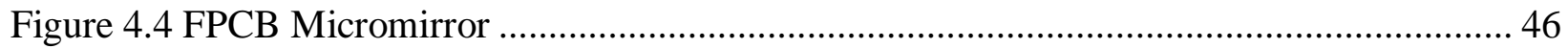

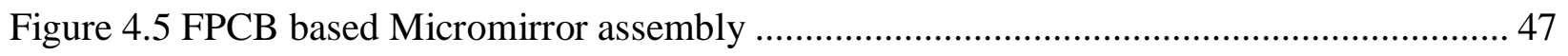

Figure 4.6 Assembly of the Laser Marking/ Engraving system ............................................. 50

Figure 4.7 LABVIEW code for the micromirror settling time .......................................... 51

Figure 4.8 Zero method code for the LABVIEW software ................................................ 52 


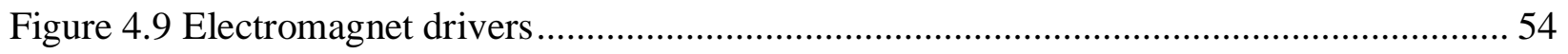

Figure 4.10 Settling time for the micromirror ..................................................................... 57

Figure 4.11 Final Prototype of Laser Marking/ Engraving System ............................................ 59

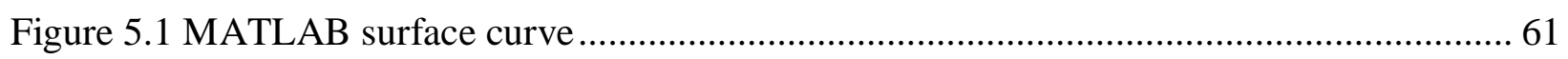

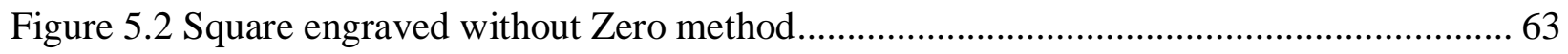

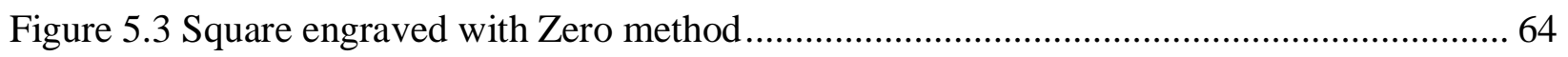

Figure 5.4 Centroid plot between engraved Square by Zero method, without Zero method and

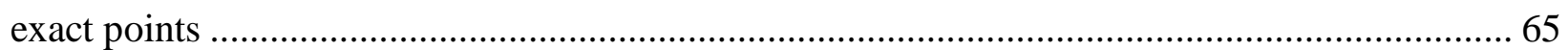

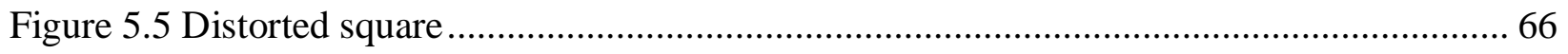

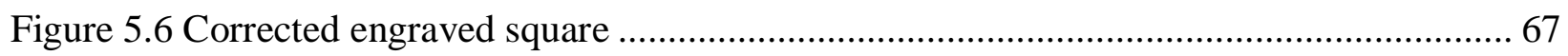

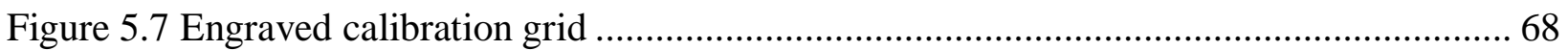

Figure 5.8 Plot between X coordinate, Y coordinate and one of the electromagnet voltages ...... 69

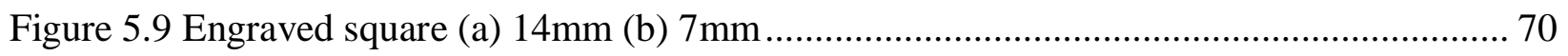

Figure 5.10 Engraved Circle (a) 14mm diameter (b) $7 \mathrm{~mm}$ diameter ....................................... 71

Figure 5.11 Jordan logo engraved (a) at higher power (b) at lower power ................................. 71

Figure 5.12 Puma logo engraved (a) at higher power (b) at lower power ................................... 72

Figure 5.13 Elephant logo engraved (a) at higher power (b) at lower power ............................. 72 


\section{List of Tables}

Table 3.1 Modelling of the FPCB based micromirror .......................................................... 22

Table 3.2 Components parameters of the FPCB based micromirror ..................................... 23

Table 3.3 Control points for the modelling of the spline spring member of the FPCB micromirror

Table 3.4 Dimension of Micromirror Seat ......................................................................... 26

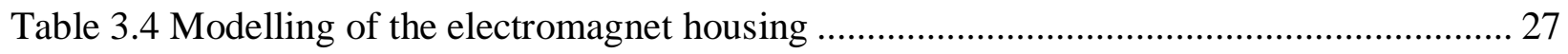

Table 3.5 Materials information used in the simulation .................................................... 30

Table 3.6 Simulated points coordinate. *Voltage values are from calibration in order to make a

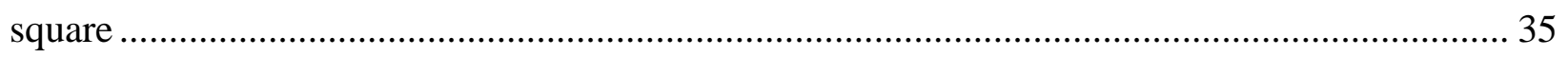

Table 3.7 Comaprision between the simulated and engraved points ...................................... 38

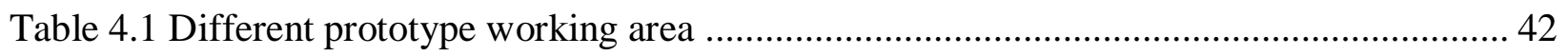

Table 4.2 Cost analysis for making a silicon mirror ............................................................ 44

Table 4.3 Experimentally engraved points coordinates ................................................... 55

Table 4.4 Power of the laser before and after striking the micromirror ..................................5 58

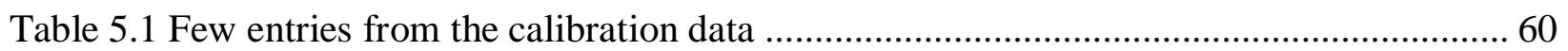




\section{Chapter 1 Introduction}

\section{Laser Engraving/Marking System}

The laser engraving/ marking system is a machine in which laser power is used to achieve engraving or marking on the desired object. Lasers are a monochromatic light beam, i.e. light beam having a single wavelength, that has enough power to change the color or ablate the surface of the target material. Depending upon the wavelength and power of the laser, the laser can be used for marking or engraving applications. These light beams can be focused to small size over longer distances which is beneficial to generate concentrated high power over that spot [1][2]. This light energy is converted into heat and can be used for different applications.

\subsection{Difference between Laser Marking and Laser Engraving}

\subsubsection{Laser Engraving}

In this process, the laser is used to make a cavity on the top surface of the workpiece. High laser power is used to remove a noticeable amount of the top layer of the surfaces. Depending upon the depth of the engraving, the engraving can broadly be classified as Etching, laser ablation, and deep laser engraving [3]. However, the main purpose is to vaporize the top surface according to the desired pattern and depth. These processes are often quite precise and multiple passes of laser beam might be done to ensure the right amount of depth is achieved over the surface of the material.

\subsubsection{Laser Marking}

Unlike laser engraving, laser marking only alter the surface property of the material. There is no physical cut over the surface and the only discoloration of the target material is achieved as a result of oxidation. The most common types of laser marking are annealing, foaming, carbon migration and coloration. As only the surficial properties are changed, there is no need for high power laser. Even the lower power laser much less than the target material irradiance limit can be utilized. It is a comparatively slower process than the previous one [3][4].

\subsection{Types of Laser Source}

Many laser sources are currently available in the market that can be utilized depending upon the application. The amount of power and its wavelength determine the effectiveness of the laser to 
that targeted material. A diode laser, crystal laser, and $\mathrm{CO}_{2}$ are available options to choose from depending on the engraving system to be designed. The diode laser is often used for lower power applications $(<5 \mathrm{~W})$ and this laser is cheaper amongst all [4][5]. Another important feature for the designing of a laser engraving system is to determine the spot size and the material absorption rate of the laser on the targeted material. This is generally governed by the wavelength of the laser. Smaller the wavelength of the laser, smaller the feature size would be [6].

\section{Electromagnetic Spectrum - Wavelength in micrometres}

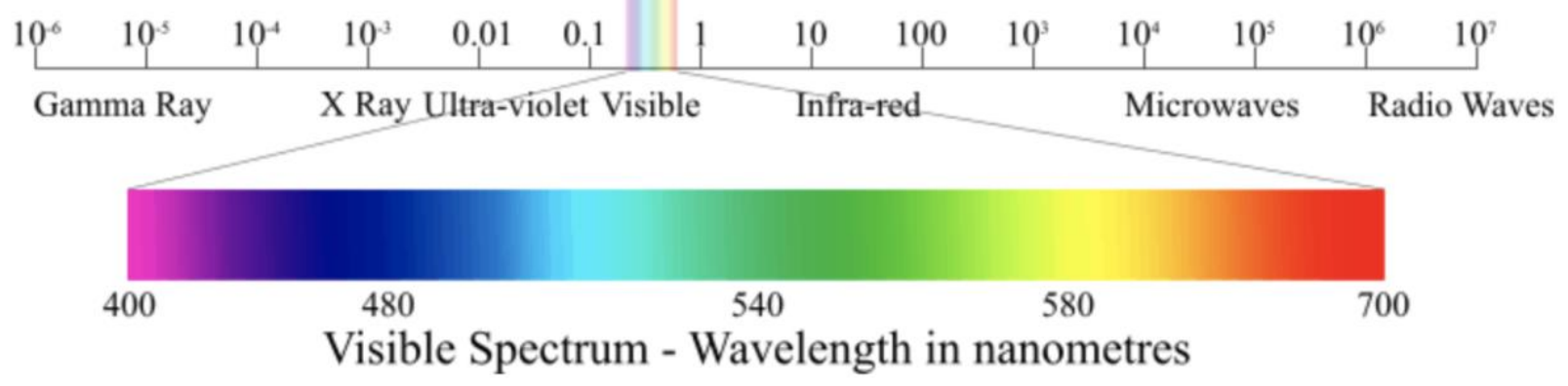

Figure 1.1 Light spectrum [7].

\subsection{Need for Laser Engraver/ Marking}

In the modern society, many industries need to distinguish their product in order to get consumers attention and increase the popularity of the products. By using laser engraving/ marking, manufacturers can monitor and trace their inventory in the warehouses. Government regulations and industrial standards also compel manufacturers to exhibit specific safety marks or logos on hazardous products and chemicals in order to ensure safety and for identification purposes. With the help of marking/engraving UDI (Unique Device Identification) numbers, barcodes, safety logos and different patterns can be created on the product which improves the traceability and control of product in the warehouse [8][9]. As an example, like for every perishable item, expiry dates and the consumable facts are needed to be marked for the consumers. This idea has now been used in many industries from automotive to medicine, textile and even in semiconductor products. All these can easily be done by using Laser marking/ engraving machines with minimal amount of time spent on engraving/ marking. High surface finish, non-contact approach, and durability are major advantage of laser marking/ engraving over other conventionally available techniques like electrochemical etching, dot peening etc. [10] [11]. 


\subsection{Laser Scanning}

For the engraving system, the laser scanner steers the laser beam to the targeted location to achieve marking or engraving. Two conventional laser scanners are as under.

1. X-Y Plotter table

2. Galvano Laser scanner

\subsubsection{X-Y Plotter Table Scanner}

In this setup, laser head travels along the rails to engrave on the desired location. The high-speed stepper motor is used and the same is connected with $\mathrm{V}$ belt. The size of the plotter table rails decides the working area. Even, the small feature can be engraved using this machine, still the size of the machine is big. Hence making it not suitable for the smaller application area. This scanner can work on a few feet with slow scanning speed [12] [13]. Its dimension and working area are $490 \mathrm{~mm} * 440 \mathrm{~mm}$ and $305 \mathrm{~mm} * 210 \mathrm{~mm}$ respectively.

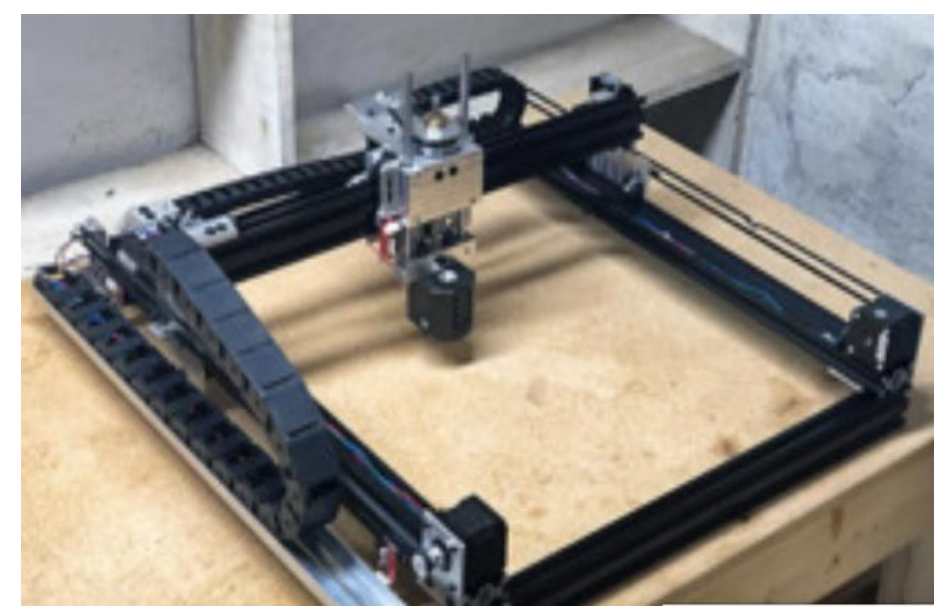

Figure 1.2 X-Y Plotter laser engraver [14] 


\subsubsection{Galvano Laser Scanner}

In this setup, the laser beam is steered on the desired area while the laser source is fixed. Highly reflective mirror plates are attached to motors (Galvanometer). Two orthogonally aligned unidirectional mirrors are needed to scan the area. Its closed loop system has a feedback position detector for minor correction, which ensures the correct position of the mirror. The feedback loop monitors the mirror movement and helps in achieving the desired orientation of the mirror. The system has some limitations, i.e. focussing a laser beam with the same intensity over a long distance is not difficult to achieve. It has a small working area. Its size is $61 \mathrm{~mm} \times 34 \mathrm{~mm} \times 43 \mathrm{~mm}$. Its driving unit is bulkier than the scanner itself of size $77.5 \mathrm{~mm} \times 65.9 \mathrm{~mm} \times 45.5 \mathrm{~mm}$. The complete system consists of two mirrors, two galvanometers, and two driving units [15]. These scanners are very expensive than a plotter table scanner. The cost of each scanner varies due to the following factors:

1. Expensive optics F-theta lens.

2. The requirement of complex driving units and feedback detector to achieve accuracy.

3. Mirrors must be chosen so that they can withstand high accelerated forces on the rotating shaft.

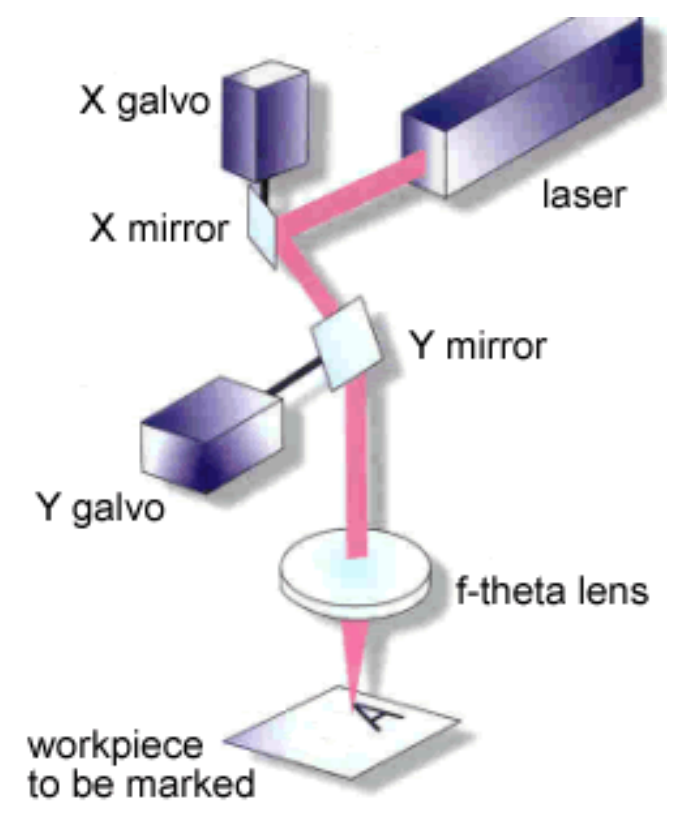

Figure 1.3 Galvano scanner laser engraver [16] 
The above mentioned two scanning methodologies are not dependable as they require regular maintenance due to the presence of numerous moving parts, i.e. motors, and servo circuits, etc. As both the scanners are bulky and expensive, hence are not advisable to be used for point and shoot operation making it unsuitable for consumer application with small area of 1 2 inch.

In order to get low cost and compact engraving machine, small size and simple structure with minimal maintenance MEMS micromirrors scanner were developed. Quality of micromirror plate must be maintained with the desired dimension and shape in order to achieve good results. Companies Mirrocle Technologies Inc and Worldstar have proposed their working prototypes in this field. Commercial MEMS used in engraving systems are as below.

\subsection{MEMS Micromirror}

These days, marking/engraving is very much needed and often used in consumer related goods like jewelry, nameplate, creating a personalized signature on wallets/belts and clothing, etc. These applications require a low-cost compact laser engraver which can be possible using MEMS micromirror in the laser engraver machine. [17-19]. For eliminating the loss of heat of laser and full reflection of incident beam, mirror plate with high flatness is required [20-22]. The quality of

micromirror depends on its fabrication process. The most conventional micromirror is not suitable for laser marking/ engraving applications. These are integrated micromirrors where the mirror and the substrate structure are manufactured together. Because of their small aperture $(<1 \mathrm{~mm})$ and low flatness (ROC Radius of Curvature $<1 \mathrm{~m}$ ), they can't be used in the engraving purposes. A new approach is emerging where the mirror and the MEMS substrate is fabricated separately. These mirrors are known as bonded micromirrors. Both actuators and mirror plates are fabricated separately and then bonded later. Large aperture of a few millimeters with high flatness (ROC $>10 \mathrm{~m}$ ) can be achieved using this method. However, the current available bonded mirrors are highly expensive (\$250 \$1000) [23][24] due to expensive microfabrication process and the low yield bonding of the mirror to the fragile actuator [25][26].

\subsection{NEXGEN Laser Marker by Worldstar Technologies}

The marker developed by Worldstar Technologies has 5-8-watt fiber laser with $1064 \mathrm{~mm}$ wavelength. One 2 directional quasi-static MEMS mirror is used for scanning of the marking area. The speed of the mirror is $20 \mathrm{~mm} /$ degree with maximum rotational angle $+/$ - 5 degrees 
(mechanical). It can engrave maximum area of $50 \times 50 \mathrm{~mm}$. Overall dimension and weight are 150x100x $25 \mathrm{~mm}$ and $1 \mathrm{Kg}$ respectively [27]. It is one of the compact laser markers, but its availability in the market is limited. Its cost is greater than 1000 USD and its scanner mirror costs around 400 to 650 USD [28].

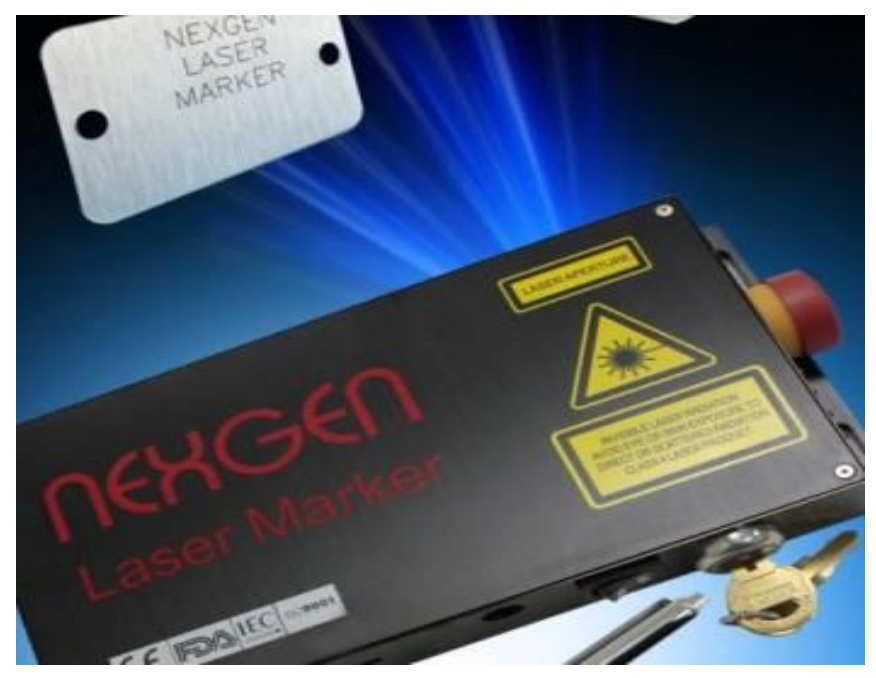

Figure 1.4 NEXGEN laser engraver [27]

\subsection{NLL10 Label Laser by Nanosec Technology Gmbh}

In this engraver developed by NanoSec Technology $\mathrm{GmbH}$, a one-watt diode laser is used operating at $405 \mathrm{~nm}$ wavelength. The speed of the engraver is 0.2 square centimeters per second and 100 millimeters per second for raster and vector scanning method respectively. Its physical dimension is $200 \mathrm{~mm}$ cube and weight is $5 \mathrm{~kg}$ with $45 \times 45 \mathrm{~mm}$ working area. Its design is simple and low cost. This marker is used for label marking on foil, leather, and plastic [29]. 


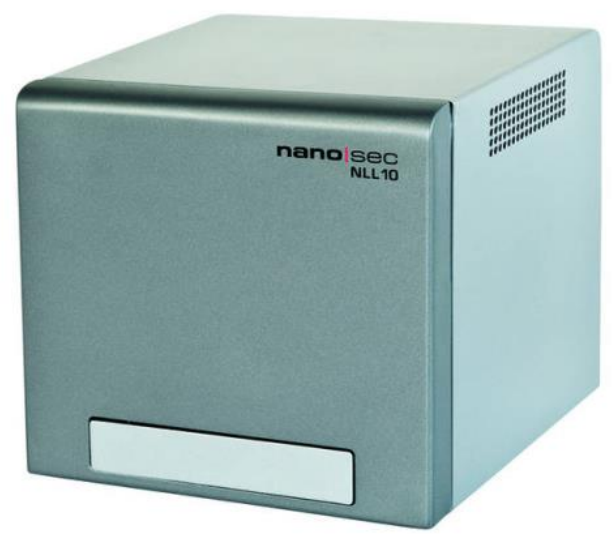

Figure 1.5 Laser engraver NLL 10 [29]

\subsection{Micromirror by Mirrorcle}

Many companies like Mirrorcle focus on developing bonded micromirrors for laser engraving/ marking. NLL10 uses the ARI-MEMS fabrication provided by Mirrorcle to fabricate two-axis scanning gimbal-less micromirror. Maximum scan angle of +/- 6 degrees (mechanical) and +/- 7 degrees (mechanical) for quasi-static operation and resonant modes respectively can be reached using single crystal silicon. Depending upon the aperture size of the micromirror, their price range varies from USD 529 to USD 1046, which is un-suitable for the consumer application point of view [29-31]. The micromirrors are expensive due to expensive microfabrication involved in the manufacturing of those mirrors. 

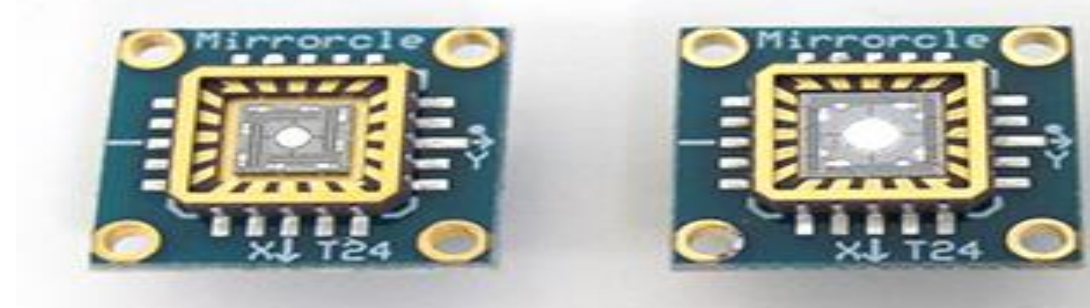

Figure 1.6 Mirrocle mirrors [31]

All these commercially available MEMS mirrors are suitable alternatives to the Galvano scanner which reduce the cost of maintenance and the overall size of the micromirror scanner. However, they are still quite expensive for the consumers making them unsuitable for the portable laser marking applications. The cost of the bonded mirrors can further be reduced significantly if the Flexible Printed Circuit Board (FPCB) is used as the member for the mirror actuation. The benefits are described in the next section.

\subsection{FPCB based Micromirrors}

Due to the expensive cost of the conventional available bonded MEMS micromirror, FPCB based bonded micromirror is one of the potential methods to overcome this limitation with comparable results. The following section will describe its benefits over conventional MEMS mirrors.

\subsubsection{Benefits of FPCB based Micromirror}

These mirrors are the type of bonded MEMS mirrors having an advantage of low cost and having comparable characteristics of the conventional mirrors. MEMS mirrors are fabricated by using the conventional microfabrication process which itself is expensive process. On the other hand, FPCB based mirrors are easily fabricated by an inexpensive, commercially available and mature process much lesser than the microfabrication process that reduces the cost significantly to few dollars ( $<10$ dollars) when mass produced. The second major advantage of using FPCB as a substrate material is that the polymide material used in FPCB has a high strain strength. The strain at a yield of the mirror to the substrate is $3 \%$ to $5 \%$ higher than that of brittle silicon $(<1 \%)$ which is used in conventional MEMS mirrors. This leads to easier bonding of the mirror with high yield and there 
is a less chance of fracture when compared to the case of releasing the brittle silicon micromirror to the fragile microactuator in the convwntional MEMS fabrication process. Both factors contribute to the low cost of the FPCB based micromirrors (few dollars/ unit) and an appropriate choice for the usage in portable laser marking/ engraving systems. This low cost will also increase the user to make full potential of the portable engraver to engrave their customized products like jewelry, wallet, marking of pen name card etc.

\subsubsection{FPCB Based Scanners for Laser Marking/ Engraving}

In order to utilize the FPCB based laser marking/ engraving, the FPCB based scanner should be fabricated so that the laser beam can be steered in the desired direction to engrave the target material. The scanner should be capable of steering the beam in the both coordinate axes i.e. the $\mathrm{X}$-axis and the Y-axis. Previously, Karlmarx et. al. has developed the FPCB based scanner system by using two 1-dimensional micromirrors. In this scanner system, each mirror is responsible to steer the laser beam in one axis. The two micromirrors are placed orthogonal to each other hence, steering the beam in both axes. The actuation of this micromirror scanner is based on the Lorentz force, i.e. the current carrying conductor experience a force when placed in a magnetic field. This requires each mirror to be placed between two magnets having a mirror installed on the FPCB substrate to actuate. So, in a total of four magnets and two mirrors are engaged in engraving a 2dimensional pattern. [32]

\subsubsection{Limitations of the Configuration of two 1-Dimensional FPCB Scanners}

The configuration of two 1-dimensional micromirror makes the structure quite complicated to use. Few limiting factors for this type of scanner are listed as follows [32]:

1. There is a loss of power of the incident laser beam due to the reflection caused by two mirrors. The two mirrors involved have the reflectivity of around $80 \%$. The power is lost due to multiple reflections and only $64 \%$ of the input power can be reached to the target material.

2. The present configuration of two 1-dimensional mirrors makes the structure little complicated to align with the incident laser beam. Also, the factor to be considered is that after reflection from the first mirror, the beam is no longer circular and there is a huge chance to project the laser spot on the periphery of the second mirror and hence causing 
the polymide substrate of the second mirror to heat up. This also limits the power to some extent.

3. The footprint size of this configuration is also larger when two mirrors are arranged orthogonally.

All these limitations can be overcome by using one 2-dimensional micromirror instead of two 1dimensional micromirrors. As the reflectivity loss by one mirror would be $20 \%$ and hence the output power after reflection of the mirror can be increased by $20 \%$, assuming no other loss also, single micromirror would have a lower footprint size and much easier to align with the laser beam. This project focuses on fabricating 2-dimensional micromirror based on this FPCB method for laser marking/ engraving purposes. Next section contains the thesis objective and the overall layout of this thesis.

\subsection{Thesis Objective}

1. Design and develop a 2-dimensional quasi-static FPCB based micromirror capable of steering the incident laser beam in 2-dimensional space.

2. Prototyping the 2-dimensional micromirror scanner and its assembly for the Laser Marking/ engraving purposes.

3. Develop a quasi-static electromagnetic FPCB based portable Laser Marking/ Engraving System using the developed micromirror for consumer related low power applications.

4. Identify the problem creep/ drifting associated with this quasi-static FPCB based micromirrors used for laser engraving/ marking system. Propose the possible solution in order to minimize or eliminate the problem. Also, experimentally verify the proposed solution.

The organization of the thesis:

- Chapter 2 deals with the design and basic principle of the actuation of FPCB micromirror. It also deals with the components used for the laser scanner and the corresponding laser engraving/ marking system.

- Chapter 3 focuses on the modelling of the entire system with the simulation of the micromirror and its actuation. 
- Chapter 4 presents the prototyping and basic experimental test results of the micromirror assembly.

- Chapter 5 deals with the identification of the new problems with this type of nonlinear micromirror and its possible proposed solutions. It also has the results of the engraved material made using the solution proposed in this chapter.

- Chapter 6 presents the summary and the conclusion of the thesis. 


\section{Chapter 2 Design of FPCB Micromirror and the Engraving/ Marking System}

Micromirror devices consist of the reflective mirror and an actuation system that help in controlling the movement of the mirror to steer the incident laser beam. There are different types of micro-actuators that are discussed in detail in this section.

\subsection{Micro actuator}

Micro actuators are one of the main parts of the micromirror devices. They actuate the mirror depending on the input energy. Various microactuators are present in the market and can be classified based on the triggering input methods:

1. Piezoelectric Actuators

2. Thermal Actuators

3. Electrostatic Actuators

4. Magnetic Actuators

\subsubsection{Piezoelectric Actuators}

These actuators are made of piezoelectric materials which convert input electric energy into mechanical energy. When the current is applied to these types of materials, they respond to the stimuli by undergoing mechanical deformation. This mechanical deformation can be utilized in micromirror devices in order to rotate the micromirror which is attached on the surface. Linear response of these mirrors (voltage-mechanical angle rotation) is an advantage over other types of actuation. Other benefits are faster response, high force density and low power consumption. However, due to complex fabrication processes and small angular displacement of the mirror for the applied input voltage makes it less favorable amongst others [33][34]. Kah How Koh et. al. fabricated a 2D micromirror based on the piezoelectric actuator that can reach up to a maximum of 2.8 degrees at $10 \mathrm{Vpp}$ applied across 6 actuators when bending mode of the micromirror is analyzed at the $122 \mathrm{~Hz}$ natural frequency [35]. 


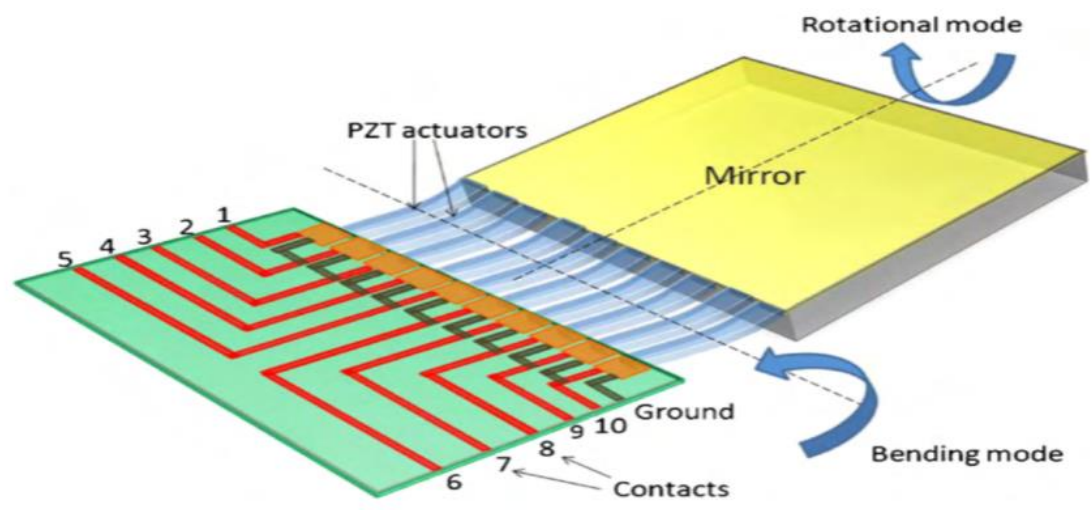

Figure 2.1 Schematic diagram of MEMS piezoelectric bending/ twisting micromirror [35]

\subsubsection{Thermal Actuators}

Thermal actuators are the substrate which undergo thermal expansion on the application of the heat. These actuators comprise of two dissimilar materials having different thermal expansion coefficients. On the application of the heat, both materials expand at different rates, hence, providing mechanical actuation. They are also known as Shape Memory alloys (SMAs) and can regain their original shape upon the removal of the heat. They are generally made of copperaluminum-nickel alloy and nickel-titanium alloy [36-38]. TiNi/ Si optical micromirror was designed and fabricated by $\mathrm{Y}$ Q Fu et al. based on the thermal actuation. These actuators are relatively easy to fabricate, however, they need high power to drive and have a slow response time. Thermal fatigue due to repeated heat cycles lowers the performance of these actuators over time.

\subsubsection{Electrostatic Actuators}

This is the most commonly preferred actuation method among all due to its fast response time $(<0.1 \mathrm{~ms})$ with minimal power consumption. Electrostatic actuators are based on the coulomb force of attraction between two charged bodies placed at some distance apart, depending upon the force and its angle. Most commonly used electrostatic actuators is parallel plate. In this actuator, two plates with different charge are placed near each other to experience charge force of attraction and repulsion. Comb drive actuator is also one of the electrostatic actuator where the attraction and repulsion are in between two members which are in proximity as the members of the comb. The latter gives the higher angle due to the torsion actuation of the beam. Wibool et al developed a 2dimensional gimbal-based scanner based on the angular comb drive actuator. They used three deep reactive ion etching process to fabricate the scanner with $+/-6.2$ degrees (at 55Vdc) and $+/-4.1$ degrees (at 50Vdc) for inner and outer gimbals respectively [39]. 


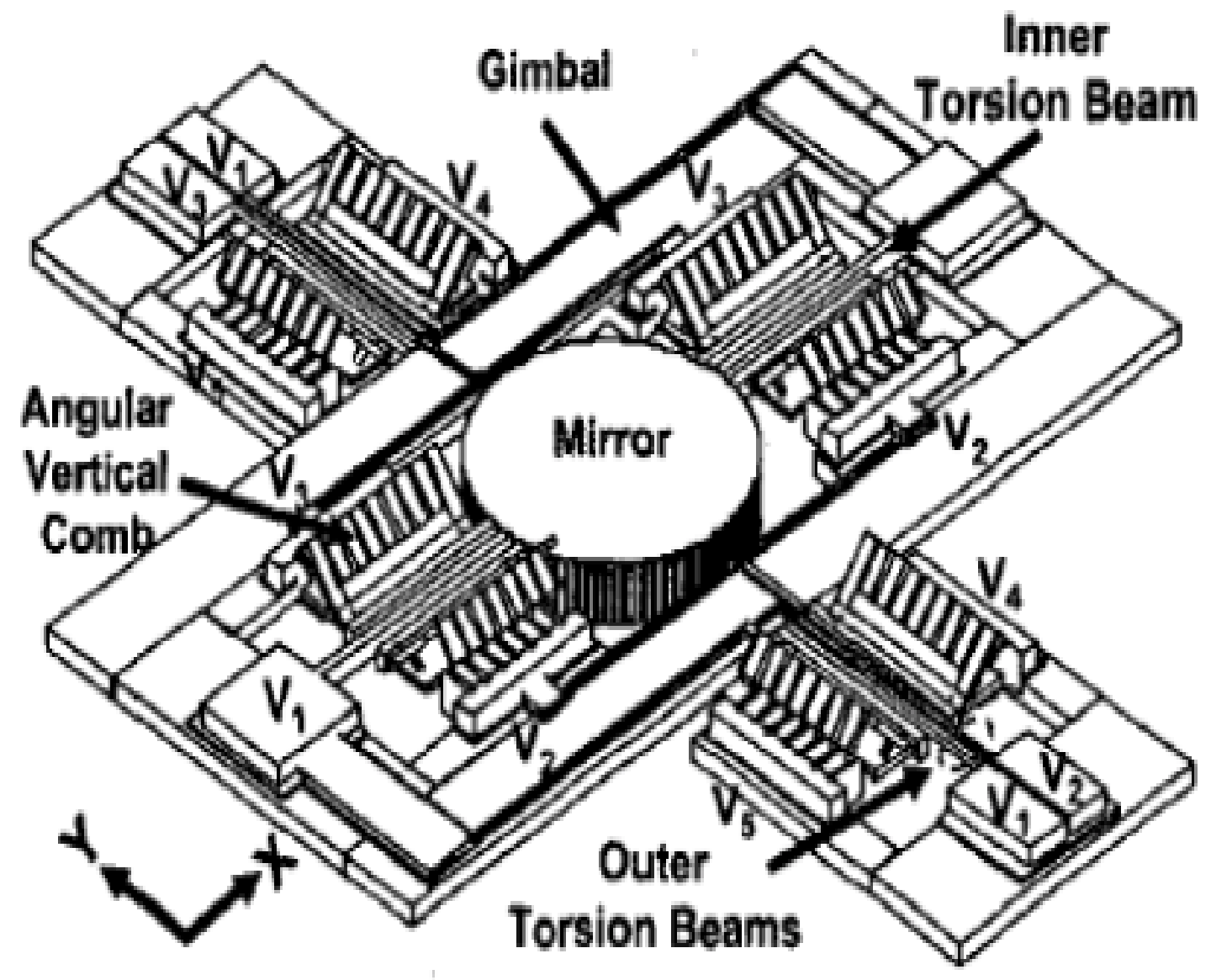

Figure 2.2 Gimbal based micromirror [39]

\subsubsection{Magnetic Actuators}

These actuators actuate the mirror by the force generated by the interference of the magnetic field. These actuators can broadly be classified into two depending upon the interaction.

1. Lorentz force: where the force is due to the current carrying conductor placed in the magnetic field. Karlmarx et. al. has developed a Lorentz force micromirror based on FPCB substrate. Two unidirectional micromirrors are placed orthogonally to steer the laser beam for bi-axial scanning. The amount of current passing through the conductor in the magnetic field determines the angle rotated by each micromirror [32]. 


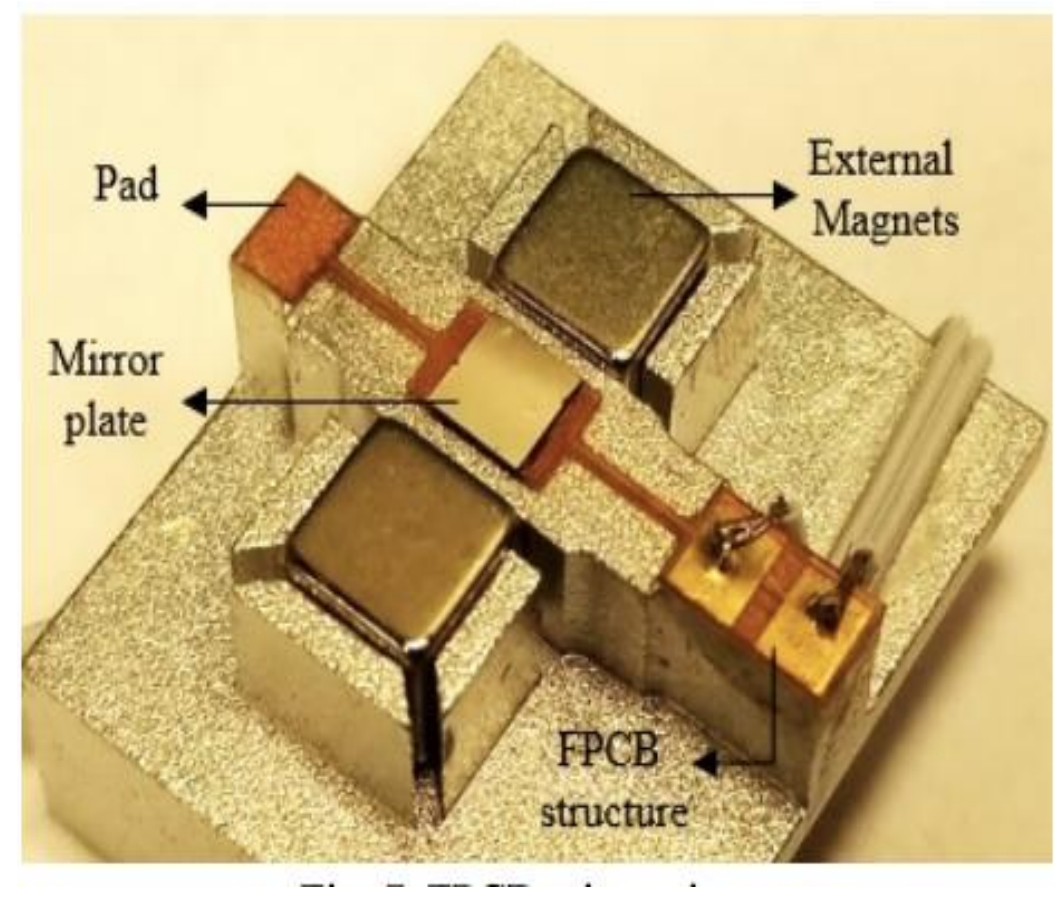

Figure 2.3 Unidirectional FPCB based micromirror [32]

2. Magnetic attraction/ repulsion-based actuators: In this type of the actuators, the magnet is bonded at the bottom of the micromirror and the electromagnet is placed underneath it. To govern the movement of the micromirror, the current is supplied to the electromagnet which either attracts or repels the magnet. Yuan et. al. has developed a similar actuator based on the repulsion force between the magnet and the electromagnet. The micromirror is tilted by the force experienced by the magnets cause of the electromagnet beneath it [63].

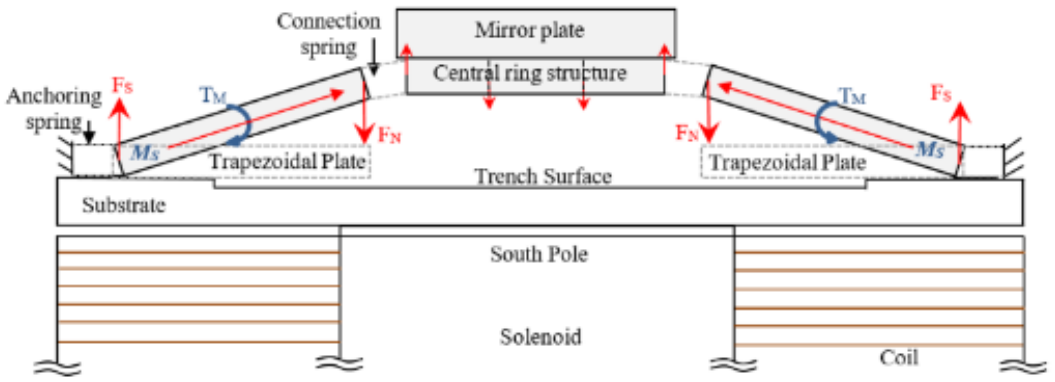

Figure 2.4 Attraction/ repulsion of magnet and electromagnet [63] 


\subsection{Design of Electromagnetic based FPCB Micromirror}

The FPCB micromirror consists of a central seat with three curved torsional beams placed at $120^{\circ}$ apart to provide the symmetry of the model. The entire model is made of FPCB i.e. copper layer sandwiched between two polymide substrate layers. The FPCB structure is fabricated using an easily available approach of cutting in the market rather than a conventional lithography process. The mirror which is fabricated by dicing the silicon wafer coated with a thin metal film is then bonded to the FPCB structure. As the strain limit at a yield of the polymide is $3 \sim 5 \%$ higher than that of brittle silicon, the bonding can be made easily and stronger as compared to the conventional MEMS mirror.

At the bottom of this seat, three tiny magnets are bonded on the curved torsional beams with the help of epoxy at $120^{\circ}$ apart. The whole structure has three curved torsional beams, $120^{\circ}$ suspended, that are supported by supporting pads (FR4 material) at the end which are screwed to the mechanical structure. The design of the beams should be such that the mirror can be tilted to the desired orientation when the bottom electromagnets are driven. Three electromagnets are placed under each magnet at a constant distance. This distance must be calculated so that the magnets do not sticks to the electromagnet at any time even while operation.

The idea behind designing the actuator is that the bonded mirror should be able to steer the incident laser beam in the two different axes i.e. $\mathrm{X}$ and $\mathrm{Y}$ axis. It is actuated by the attraction/ repulsion forces generated between the magnets and the electromagnets beneath them. The magnets are glued at the bottom of the mirror which experiences the force of attraction and repulsion by the electromagnets kept at some distance from the magnets. Detailed micromirror modelling with its specifications will be discussed in the later chapters.

\subsubsection{Working of the Electromagnetic Attraction/ Repulsion based FPCB actuator}

The basic principle of the electromagnet is to generate a magnetic field when the current is passed through the coil of wires. The strength of the electromagnet can be increased by placing a ferrite material (a material having a high permeability) in the center of the coil and the polarity can be controlled by the current direction in the wires of the coil. This electromagnet can be utilized in the movement of the micromirror. The movement of the micromirror having three magnetelectromagnet pair can be explained as follows. The three magnets are glued at the bottom of the 
curved torsional beams, beneath them the electromagnets are placed. As the magnets are fixed, the same pole is always facing towards the electromagnet at all times. When the current is passed in the electromagnet, depending on the direction of the current, the electromagnet will either attract or repel the magnets attached to the FPCB structure placed above it. In order to govern the movement of the mirror, any two of the magnets should be moved opposite to the third one. This also ensures that there is a minimal resultant force generated which is responsible for the translation of the micromirror. Depending upon the angle to be reached the right combination of the three current should be supplied to the electromagnet.

\subsection{Magnet Selection for the Model}

As the magnets are to be glued at the bottom of the FPCB structure, they shouldn't be too much heavy, however, they should be powerful enough to respond to the magnetic field generated by the electromagnet placed beneath them. The following factors are considered before selecting:

1. Weight: Heavier magnet will increase the response time of the mirror to the actuation mechanism at the expense of the tilting angle of the mirror.

2. Shape: Magnet could not be cube due to the sharp edges that might cause stress concentration where they are glued when the mirror is tilted. Disc magnets are preferred.

3. Strength: High strength magnet is needed to increase the forces responsible for the movement of the mirror. N52 neodymium magnets are preferred.

Disc magnet of size $1 \mathrm{~mm}$ diameter and $0.5 \mathrm{~mm}$ thickness was chosen to glue on the bottom of the curved torsional beams.

\subsection{Selection of the Electromagnets}

This is the main component responsible for the actuation of the micromirror. There are many factors associated with the selection of the electromagnet. For the current prototype, the electromagnet is customized. Several factors which contribute to the performance and the designing of the electromagnet are analyzed.

1. Number of turns: More the number of turns, higher would be the magnetic field generated.

2. Length of the coil: Increase in length of the coil decreases the strength of the magnetic field of the electromagnet.

3. Core Material: Higher permeable material enhances the magnetic field accordingly. 
4. Current: It is the driving factor for the electromagnet.

However, one important factor that is to be considered before designing is the heat generated by the coil wires when the current is passed through them. The wires of the electromagnet are so closely packed that applying current exceeding the certain limit will either fuse the conducting wire or permanently magnetized the core material which in turn destroys the purpose of the electromagnet. Depending on the design data, $19 \mathrm{~mm}$ long electromagnet with 1600 turns and $6 \mathrm{~mm}$ outer diameter with the soft iron core is selected for this prototype.

\subsection{Design of FPCB based Scanner}

Magnets are installed at the bottom of the FPCB structure with a silicon mirror on the central seat. The electromagnets were placed beneath the magnet. The whole setup is aligned in such a way that the mirror surface is vertical to the striking laser beam.

\subsection{Micromirror based Laser Engraving System}

Laser and its optics also play a vital role in any laser engraving machine. It decides the precision and repeatability characteristic of the engraving system.

\subsubsection{Laser Source}

$445 \mathrm{~nm}$ diode laser having a maximum power of $1.6 \mathrm{~W}$ is used in this project. This laser has a shorter wavelength and falls in the visible region (blue), which is ideal for the small spot size and current application [41]. As it must be made portable and directly consumer oriented, the cost is mostly one of the crucial factors. This leads to choosing the diode laser over the fiber or $\mathrm{CO}_{2}$ laser which are costlier than the diode laser. Depending upon the laser scanning mechanism used for the engraving or marking purposes, the laser should have the provision to accommodate the analog or the continuous beam feature. The laser used has both the functionality for the beam output and can be controlled using a NI multifunction DAQ which is described in the later sections. It works well for the tested materials of low material toughness. Also, the power for which the engraver system can engrave also depends on the amount of the power the micromirror can withstand. As the scanning micromirror substrate is polymide based which has limitations to heat, the maximum power must be calculated for which the polymide substrate can withstand the heat produced. It is described in the following chapters. In the model, there is a provision to change the laser source depending on the necessity of the application. 


\subsubsection{Optics for the Laser Source}

Pre-installed focus, that comes with the laser module is used to focus the beam at the target material. The conventional laser engravers in the market are often using the F-theta lens to achieve the focus for the laser. The biggest importance of using F-theta lens is that it increases the precision and the working area of the engraving system [42][43]. It uses the concept that the output beam is displaced by theta (angle of the incident ray) times the focal length. This provides a linear relationship between the mirror movement and the reflected ray, hence providing better control for the users. Also, the F-theta lens is being used where two one-dimensional mirrors are used to guide the incident laser beam. However, for the current prototype, the cost and size are one of the important factors and the cost of F-theta lenses are few hundred USD. Also, as the idea is to make the scanner footprint size smaller, the F-theta lens itself has a size of typical $85 \mathrm{~mm}$ which would affect the significance of the current model.

Due to the above said factors, the choice for the optics is now narrowed to basically two different types present in the market [44]:

1. Multiple Element Lens: As the name suggests, there are optics having more than one lens placed in combination to achieve the focus at the desired distance. However, due to multiple lenses, there is a loss of power, but better steering capabilities of the laser beam with the smallest spot size when compared to other optics.

2. Single Element Lens: Also classified as G series lens. (G1 to G7). They are singlet lens. There is low power loss of the laser at the cost of beam characteristics.

The prime concern is to have better control of the laser, the multiple element lenses, which was pre-installed with a laser is used for the engraving/ marking purposes in this prototype.

\subsection{Micromirror Scanning Modes}

Micromirror scanner with the FPCB structure and the electromagnets are placed at $45^{\circ}$ to the laser source to have the highest possible reflectivity of the mirror. With this mirror, scanning can be done in both the axes and engraving/ marking can be done in all the four quadrants. There are two different modes on which the current engraver scanners in the market are working: Raster mode and Vector mode. The scanning mode will determine the total engraving time and the precision of the end-product. This prototype is focused to work on vector mode as it is ideally designed for the quasi-static mode of the FPCB based micromirror to minimize the total engraving time. 


\subsection{Chapter Summary}

In this chapter, different actuators are discussed and analysed that are widely used in the market. A new design for a 2-dimensional micromirror is proposed which is capable of steering the incident laser beam on both the axis i.e. the $\mathrm{X}$-axis and the $\mathrm{Y}$-axis. It also deals with the various parameters that has to be taken into consideration for the upcoming modelling and prototyping of the micromirror scanner.

The essential design parameters for the entire laser marking/ engraving system is also briefed in this chapter. This gives a general idea for the upcoming prototyping of the scanner and its application in laser marking/ engraving system. 


\section{Chapter 3 Modelling and Simulation of the FPCB Micromirror based Engraving System}

\subsection{Modelling of FPCB Structure}

The modelling of FPCB and its mechanical support parts is done using SolidWorks software. The Flexible Printed Circuit Board (FPCB) structure consists of two layers of flexible polymide substrate and the rolled annealed copper layer in between them. The FPCB structure has a central plate for the micromirror to be mounted. This central plate has a hole in the center and is supported by three torsional FPCB beams designed in such a manner that the mirror can steer the incident laser beam in 2-dimensional space. All the three supporting torsional beams have a supporting pad with a central hole, and it is made up of Flexi-Rigid material, commonly used for PCBs, (FR4) material. The hole in the supporting pads is made to allow the user to screw the FPCB structure to the mechanical parts fabricated. The central plate hole is used to align the micromirror exactly in the center. The overall thickness of the FPCB current prototype is about $0.14 \mathrm{~mm}$. The modelling and the fabrication of the micromirror are relatively easier as there is no circuitry involved in this model. The FPCB material is used as the mechanical member to provide rotation of the micromirror. Three magnets are attached at the bottom of the torsional beams subtending from the central plate. These magnets are attached in such a way that the distance of each magnet from the center is constant. This is to ensure the symmetry in the model. The mechanical parts are also designed in order to have the housing of the three electromagnets and the FPCB structure. Proper alignment and the arrangement of the micromirror scanner is described in the next chapter. Below is a table having all the dimensions of the FPCB structure. Later mechanical parts are also described. All the values in the table are in $\mathrm{mm}$. 
Table 3.1 Modelling of the FPCB based micromirror

Dimension of the entire FPCB actuator

\section{Mirror plate}

\begin{tabular}{|c|c|}
\hline Length of the side (a) & Thickness (b) \\
\hline 4 & 0.15 \\
\hline
\end{tabular}

FPCB overall dimension actuator film

\begin{tabular}{|c|c|c|c|}
\hline \multicolumn{2}{|c|}{ Diameter of the entire model (c) } & \multicolumn{2}{|c|}{ Thickness of the entire film (d) } \\
\hline \multicolumn{2}{|l|}{13.75} & \multicolumn{2}{|r|}{0.14} \\
\hline \multicolumn{4}{|c|}{ FPCB individual section } \\
\hline $\begin{array}{l}\text { Center plate Diameter } \\
\text { (e) }\end{array}$ & $\begin{array}{r}\text { Tors } \\
\text { mec }\end{array}$ & & $\begin{array}{l}\text { Support pads (3 nos identical) } \\
\text { Square side (f) }\end{array}$ \\
\hline 5 & & & 5 \\
\hline \multicolumn{2}{|c|}{ Radius of the fillets used near the FR4 Pads (g) } & \multicolumn{2}{|c|}{$\begin{array}{l}\text { Radius of the fillet near center plate } \\
\text { (h) }\end{array}$} \\
\hline \multicolumn{2}{|c|}{1.5} & \multicolumn{2}{|r|}{0.25} \\
\hline \multicolumn{2}{|c|}{ Diameter of the center go through hole (i) } & \multicolumn{2}{|c|}{ Diameter of the hole at the FR4 (j) } \\
\hline \multicolumn{2}{|c|}{1.2} & \multicolumn{2}{|r|}{2.2} \\
\hline \multicolumn{2}{|c|}{$\begin{array}{l}\text { Torsion beam subtending angle with each other } \\
\qquad \text { (degrees) }\end{array}$} & \multicolumn{2}{|c|}{$\begin{array}{l}\text { Width of the torsion beam }(\mathrm{k})(3 \text { nos. } \\
\text { identical })\end{array}$} \\
\hline \multicolumn{2}{|c|}{120} & \multicolumn{2}{|r|}{0.5} \\
\hline
\end{tabular}


Table 3.2 Components parameters of the FPCB based micromirror

\begin{tabular}{|c|c|c|c|c|}
\hline \multicolumn{5}{|c|}{ Thickness of individual layers of FPCB film (excluding adhesive) } \\
\hline \multicolumn{3}{|c|}{ Layer name } & \multicolumn{2}{|c|}{ Thickness } \\
\hline \multicolumn{3}{|c|}{ Base substrate Polymide } & \multicolumn{2}{|c|}{0.061} \\
\hline \multicolumn{3}{|c|}{ In between Copper layer } & \multicolumn{2}{|c|}{0.018} \\
\hline \multicolumn{5}{|c|}{ Magnets (3 nos.) } \\
\hline Grade & \multicolumn{2}{|r|}{$\begin{array}{l}\text { Type and } \\
\text { Material }\end{array}$} & Diameter & Thickness \\
\hline N52 & Dis & / Neodymium & 1 & 0.5 \\
\hline \multicolumn{5}{|c|}{ Electromagnet Dimension } \\
\hline \multicolumn{3}{|c|}{ Outer diameter of the coil } & \multicolumn{2}{|c|}{ Length of the electromagnet } \\
\hline \multicolumn{2}{|l|}{6} & & \multicolumn{2}{|c|}{19} \\
\hline
\end{tabular}

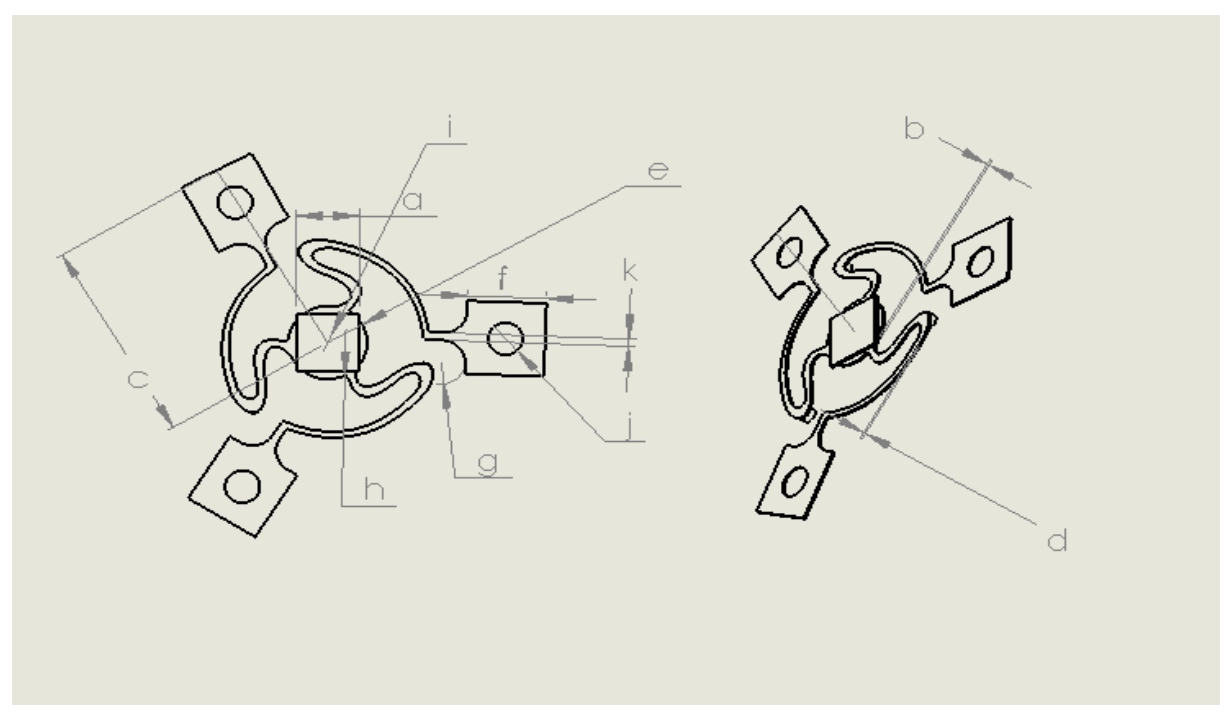

Figure 3.1 Top and Isometric view of FPCB micromirror 
The torsion beam of the FPCB structure has a spline contour and below are the control points for one of the spline. All the torsion beams are identical and subtend $120^{\circ}$ with each other.

Table 3.3 Control points for the modelling of the spline spring member of the FPCB micromirror

\begin{tabular}{|c|c|c|}
\hline Point Number & X-coordinate & Y-coordinate \\
\hline 1 & 6.36 & 0.92 \\
\hline 2 & 0.16 & 6.48 \\
\hline 3 & -1.78 & 4.6 \\
\hline 4 & 0 & 3.36 \\
\hline 5 & 1.47 & 3.40 \\
\hline 6 & 2.08 & 5.07 \\
\hline 7 & 0.18 & 4 \\
\hline 8 & 4.68 & 0.43 \\
\hline 9 & 6.04 & \\
\hline
\end{tabular}




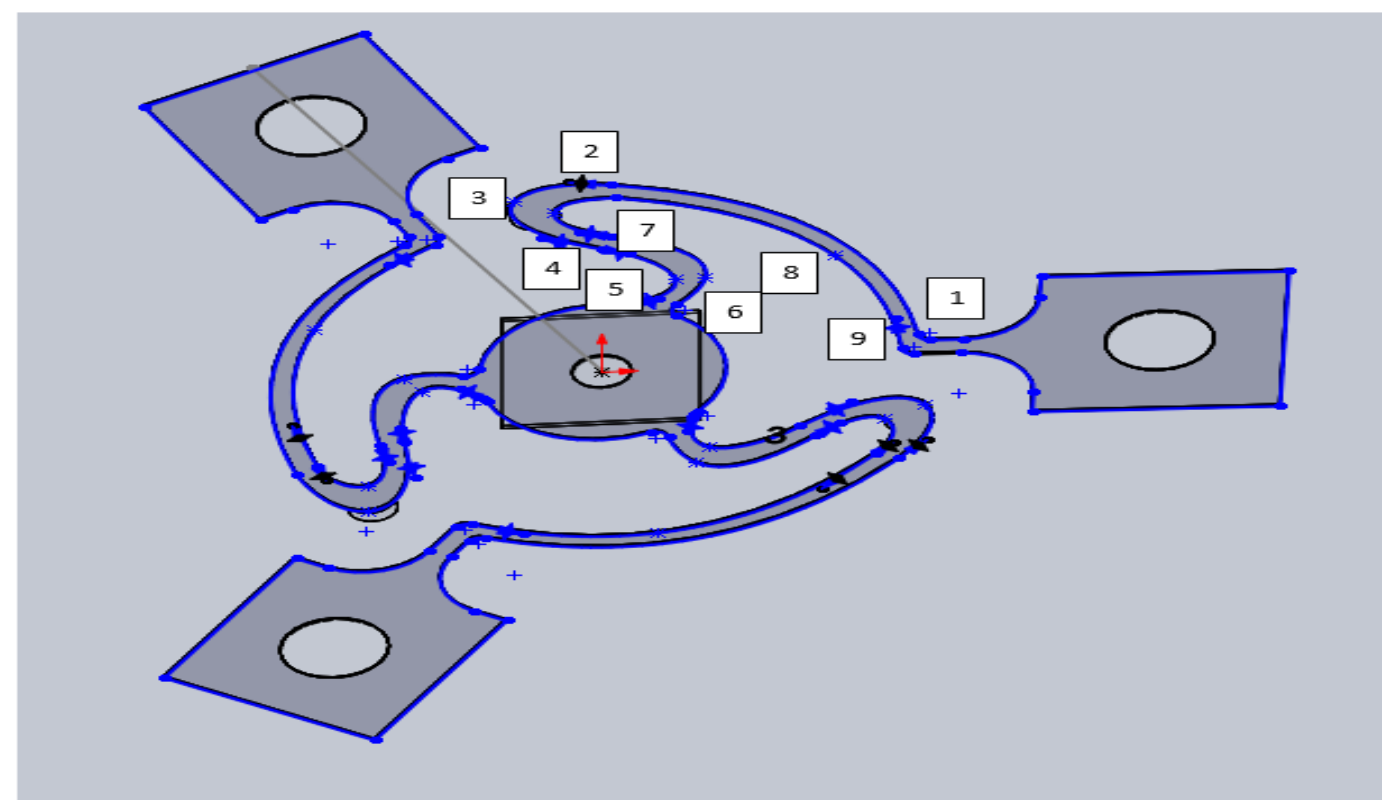

Figure 3.2 Control points of the spline of the FPCB micromirror

There is no circuit in the copper layer. The copper layer is an exact replica of the cover and substrate layer polymide. This also reduces the cost of fabrication.

\subsection{Modelling of the Mechanical Support Structure for the Micromirror}

Now the FPCB is modeled and the size of the magnet is determined. The mechanical part is to be fabricated for the support and assembling of the micromirror scanner. It should be noted that the FPCB structure should be stretched properly before assembling and the magnets attached at the bottom of the micromirror are at a constant distance of $3 \mathrm{~mm}$ from the electromagnet.

\subsubsection{Modelling of the Micromirror Seat}

In order to have a steady FPCB model for the scanning, the mechanical structure is fabricated for the support. Below is the modelling of the micromirror seat with its dimensions. Only the important dimensions are marked in mm below. Detail design is attached in the Appendix. The thickness of the micromirror seat is essential as this will determine the gap between the magnet and the electromagnet. In order to place the magnets and electromagnets at the desired distance, the 
micromirror seat with different thicknesses were fabricated. All the units used in the tables are in $\mathrm{mm}$, unless specified.

Table 3.4 Dimension of Micromirror Seat

\begin{tabular}{|c|c|c|}
\hline \multicolumn{3}{|c|}{ Dimension of the Micromirror Seat } \\
\hline Length (a) & Width (b) & Thickness (c) \\
\hline 30 & 30 & 3 \\
\hline
\end{tabular}

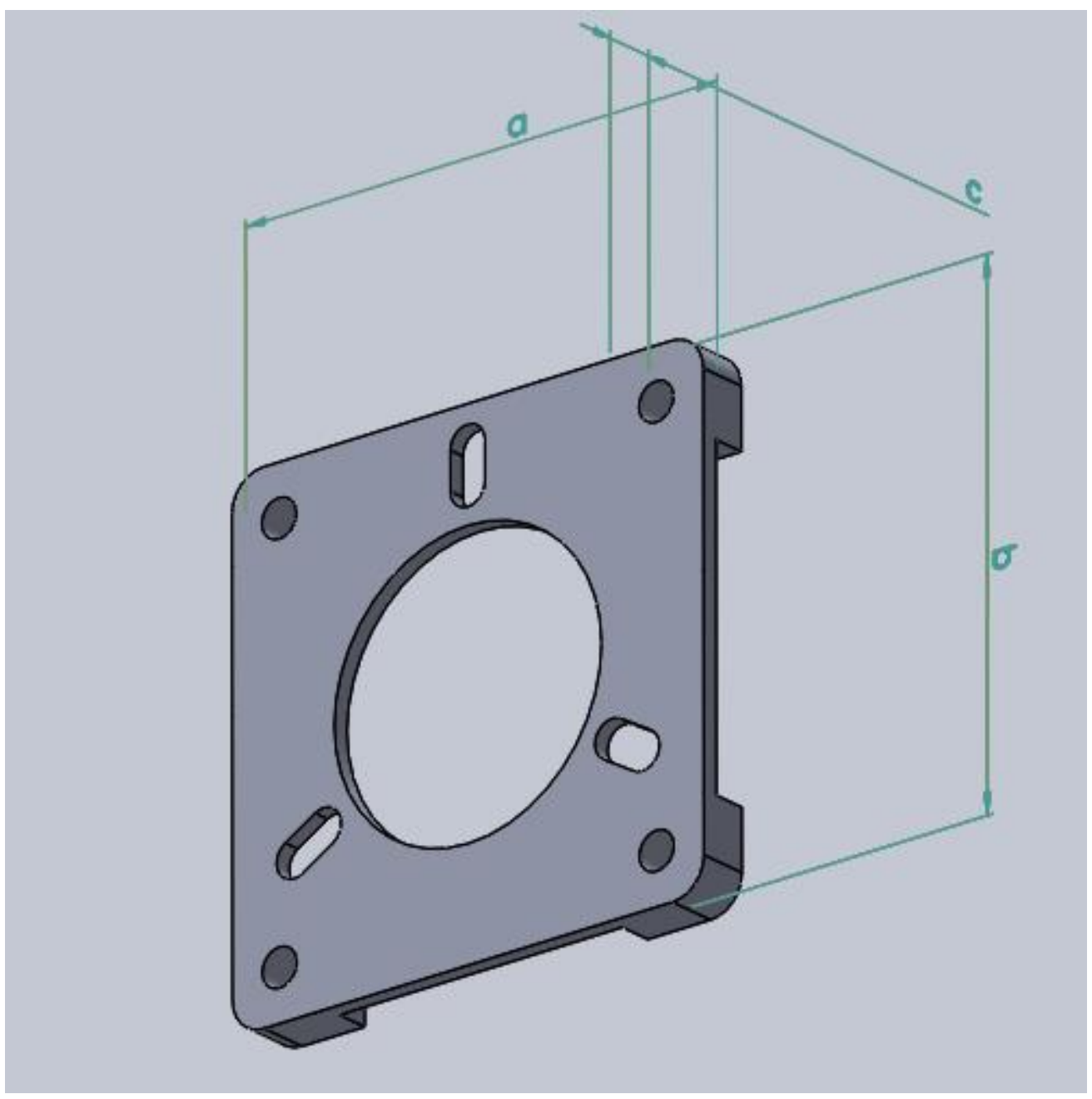

Figure 3.3 Micromirror seat modelling 


\subsubsection{Modelling of the Electromagnet Support Structure}

Electromagnets should be placed vertically and in the exact same distance apart from the central axis as that of the magnet. The electromagnets must be placed exactly at the bottom of the magnets in order to actuate the micromirror. Below are the dimensions of the various parts used in the support structure of the electromagnet. Disc structures are used so that the electromagnets can be rotated and can make sure the electromagnets are right under the magnets. All units are in mm.

Table 3.4 Modelling of the electromagnet housing

\begin{tabular}{|c|c|c|c|}
\hline \multicolumn{4}{|c|}{ Dimension for the disc to support electromagnets } \\
\hline Diameter of the disc & Distance (a) & Diameter of the hole for electromagnet to go in \\
\hline 26 & 6.4 & 6.2 \\
\hline \multicolumn{4}{|c|}{ Dimension for the support of disc Front End of the assembly } \\
\hline Length (b) & Width (c) & Height (d) & Diameter Hole for disc to slide into \\
\hline 30 & 7 & 30 & $26+0.15$ \\
\hline
\end{tabular}

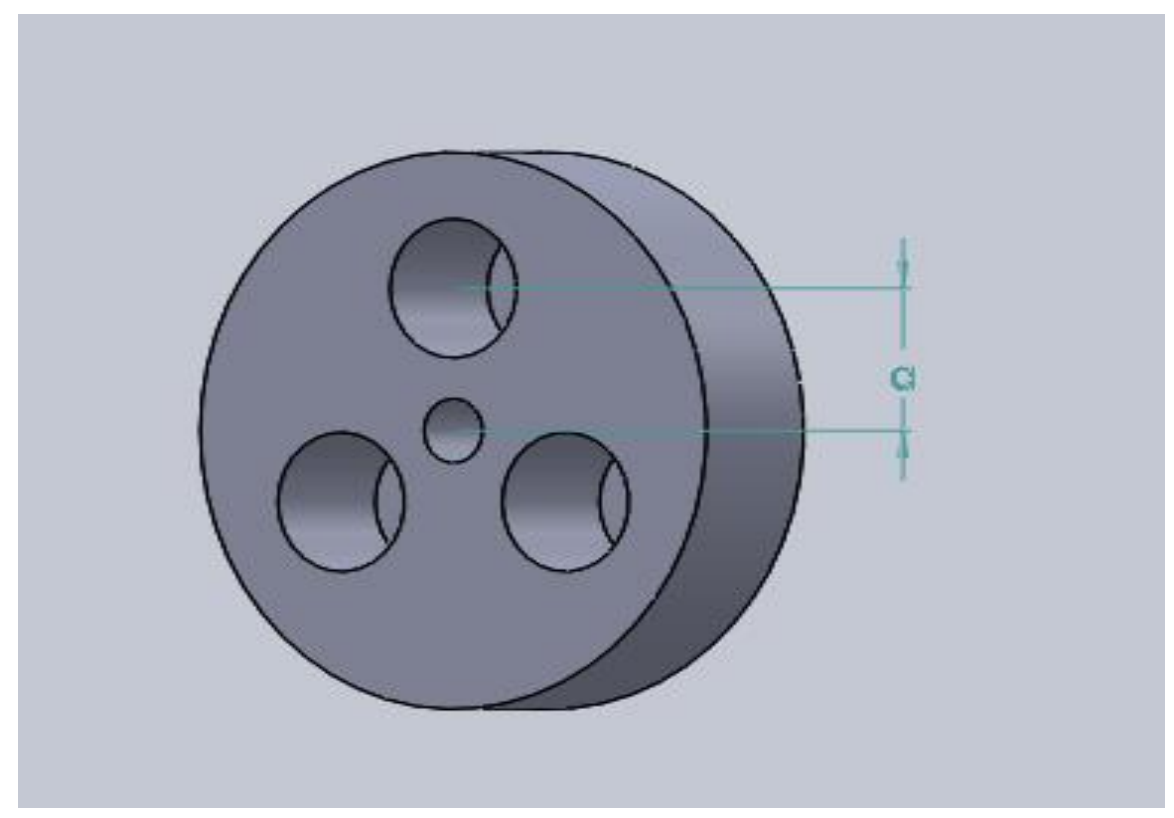

Figure 3.4 End disc of the electromagnet housing 


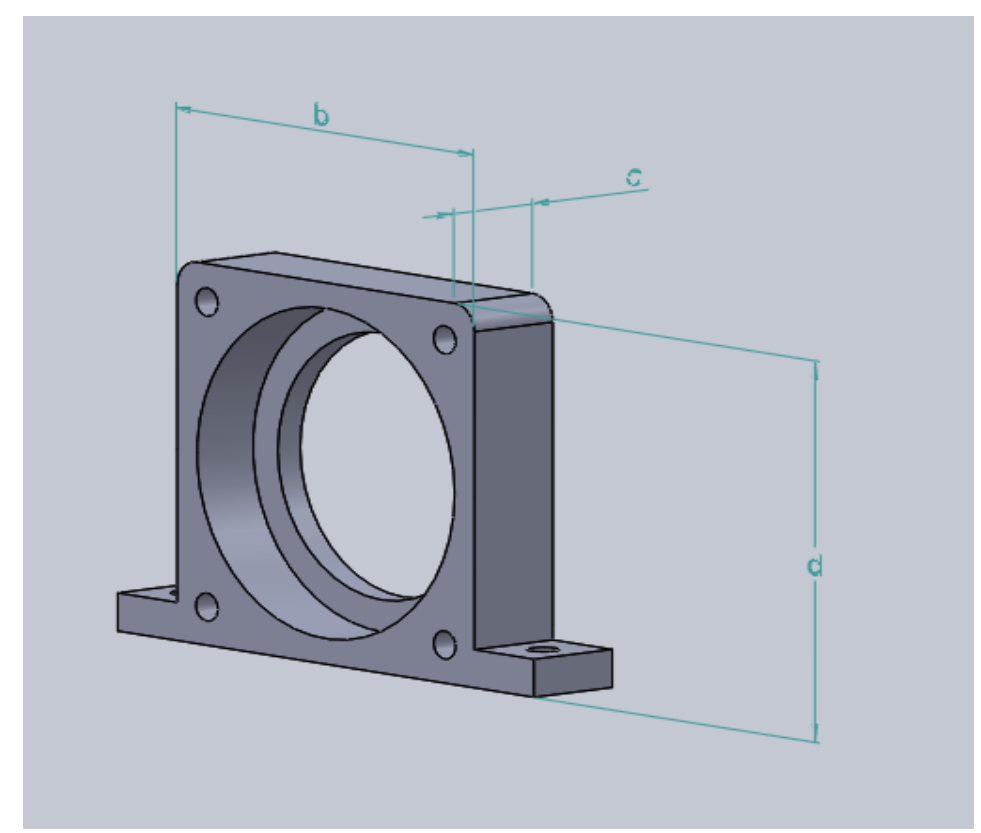

Figure 3.5 Front-end for the electromagnet micromirror scanner

\subsubsection{Modelling of Micromirror Base Plate}

The base plate of the micromirror has a grove cut-out in the body. This is to help to align the micromirror scanner along the laser beam.

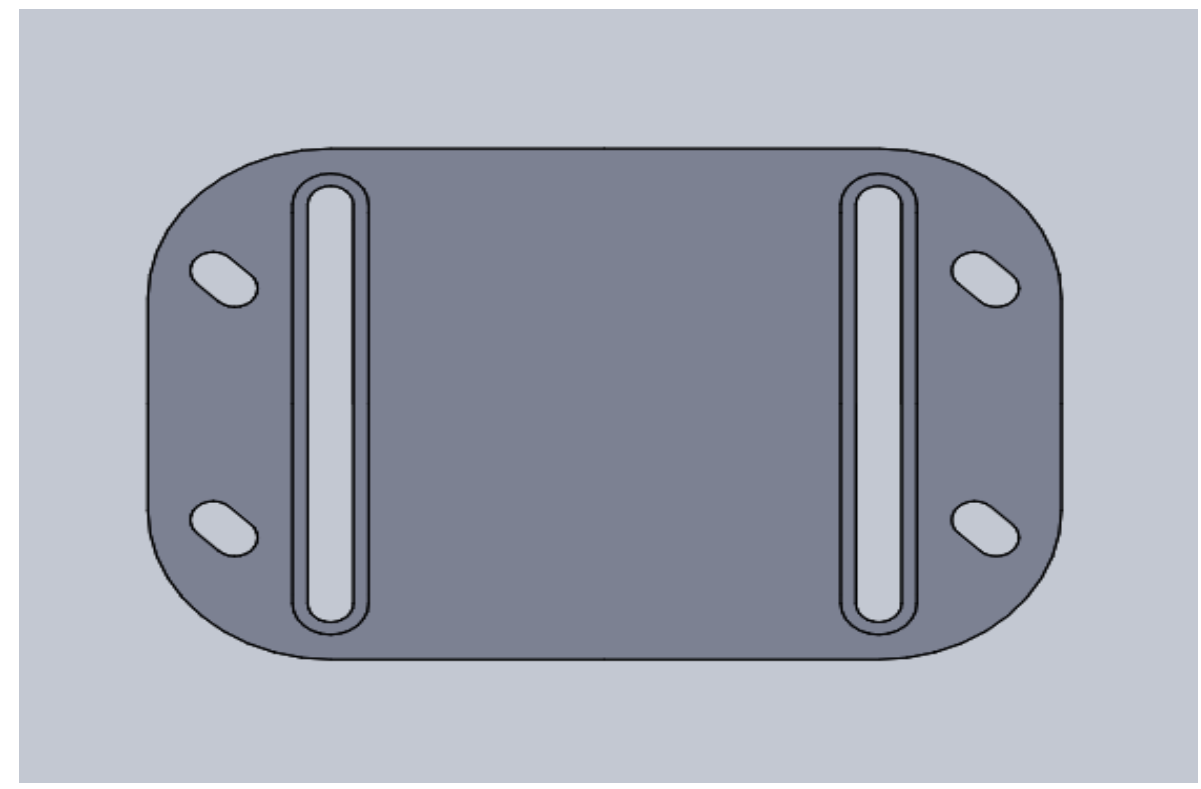

Figure 3.6 Base plate for the micromirror 


\subsubsection{The Assembly of the Laser Marking/ Engraving Micromirror Scanner}

In order to ensure that all the parts are modeled correctly, the SolidWork assembly is made and once, ensuring all the dimensions and tolerances, the parts are sent out for the fabrication. Below is the assembly of the micromirror scanner.

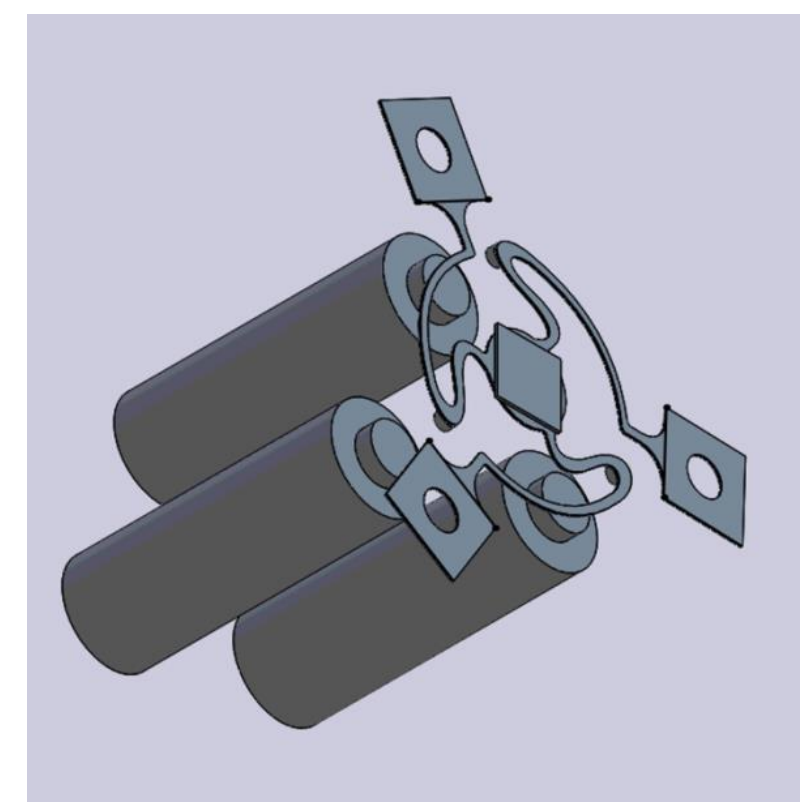

Figure 3.7 Micromirror assembly

\subsection{Simulation of the FPCB Micromirror}

After the modelling of the micromirror and its mechanical parts, simulation is carried out in ANSYS 18.1 and Maxwell. The mechanical structures are not included in the simulation as they will increase the simulation calculation and execution time. Material properties of polymide are added in the ANSYS as they are not available in the ANSYS database. Next section contains the properties of different material used. 
Table 3.5 Materials information used in the simulation

\begin{tabular}{|c|c|c|}
\hline Part Name & Material Used & Properties \\
\hline Mirror plate & Silicon anisotropic & Available in ANSYS 18.1 \\
\hline Polymide & $\begin{array}{c}\text { Kapton material properties } \\
{[47]}\end{array}$ & Described in next section \\
\hline $\begin{array}{c}\text { Rolled Annealed Copper } \\
\text { Layer }\end{array}$ & Rolled Annealed Copper & Described in next section \\
\hline Magnets & N52 grade magnet & Available in Maxwell \\
\hline Electromagnet core & Iron & Available in Maxwell \\
\hline Electromagnet coils & Copper & Available in Maxwell \\
\hline
\end{tabular}

The simulation is divided into two steps. In order to calculate the force experienced by the magnets generated by electromagnets, Maxwell software is used to simulate the results. Once the force is calculated on each of the three magnets attached to the FPCB structure, then that force is applied to the FPCB structure in the structural analysis in ANSYS 18.1.

\subsubsection{Polymide Properties}

Polymide data is acquired from the manufacturer Dupont chemicals [47]. The following material properties are used in the simulation.

Young's Modulus : $\quad 4 \mathrm{GPa}$

Poisson's ratio $\quad: \quad 0.34$

Density $\quad: \quad 1420 \mathrm{Kg} / \mathrm{m}^{3}$

\subsubsection{Rolled Annealed Copper Properties}

Rolled annealed copper is often used in FPCB structures as actuators to provide better flexibility in the model. The properties are as follows [48]:

Young's Modulus : $120 \mathrm{GPa}$

Poisson's Ratio $\quad: \quad 0.34$

Density $\quad: \quad 8970.3 \mathrm{Kg} / \mathrm{m}^{3}$ 


\subsubsection{Magnets Properties}

Magnets properties are available in the Maxwell software. However, in the prototype, the magnet forms the part of the micromirror scanner. Hence, its properties are needed to be added to the ANSYS simulation when the natural frequency of the model is simulated.

Yong's Modulus : $\quad 160 \mathrm{GPa}$

Poisson's Ratio $\quad$ : $\quad 0.24$

Density $\quad: \quad 7.5 \mathrm{~g} / \mathrm{cm}^{3}$

\subsection{Assumptions for the Simulation}

The following assumptions are made while simulating the results.

1. There is limited information on the material property of the polymide. Even the manufacturer has limited information and not sure on the specifications provided as these materials are seldom used as structural members. The property of the polymide is assumed to be isotropic and the data provided by the manufacturer is assumed to be correct.

2. In the simulation, the adhesive is not considered between the layers of the FPCB structure. There is no data on the amount of epoxy used in order to glue the layers. This factor might affect the simulation data.

3. Magnets are assumed to be in the same plane and all the three magnets are at a constant distance from the electromagnets i.e. $3 \mathrm{~mm}$.

4. The FPCB structure is perfectly straight and there is no slackness in the structure.

5. For the magnetic simulation, the surrounding shell around the model is $150 \%$ bigger than the model itself and assuming the magnetic field lines are well inside this domain.

\subsection{Static Simulation}

The static simulation is broadly divided into two steps. Static structural simulation is done in ANSYS Static simulation and magnetic simulation is done in Maxwell.

\subsubsection{Maxwell Magnetic Simulation}

For this simulation, the three magnets with three electromagnets are modeled in Maxwell. The distance between the magnets and the electromagnets is kept constant. In order to save the execution time of the simulation, only magnets and electromagnets are imported in the simulating 
software. It is assumed in this simulation that the magnets are experiencing virtual force generated by the electromagnets. As the force is virtual, there is no deformation/movement in the simulation. Hence, there is no need for importing the whole model as it is also assumed to be fixed in one position. The current is initialized in the electromagnet. Depending upon the direction of the current, as the magnet face is fixed towards the electromagnet, the magnet will experience either force of attraction or repulsion. The directional forces on each of the magnet are noted for the structural calculation of the model. Below is the figure in which the magnet is experiencing $0.1 \mathrm{mN}$ repelling force from the electromagnet. Several combinations of current values are applied, and the force combination is stored in excel sheet for further analysis.

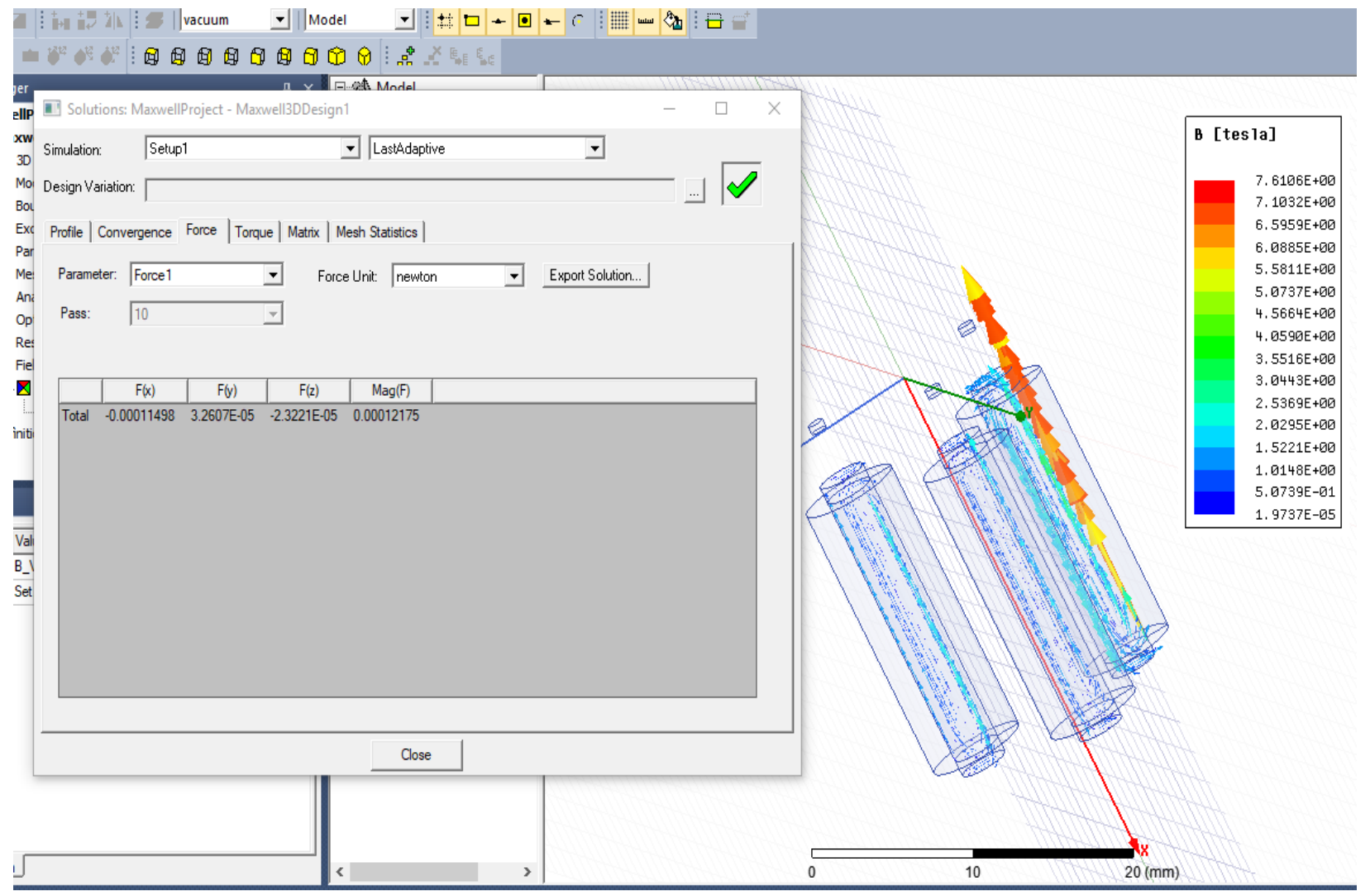

Figure 3.8 Maxwell magnetic simulation 


\subsubsection{Static Structural Analysis}

In this simulation, the force from the magnetic simulation is applied to the structural model of the prototype. The electromagnets are not present in this model and only FPCB structure without magnets is imported. As the magnet themselves experienced the force, it is assumed that magnet is causing the deflection of the structure, hence excluded from the simulation. The force applied to the body generates some deflection in the body and the deflection caused is noted down for finding the laser beam spot on the target material. The deflection caused on the beam is calculated and stored in the excel sheet.

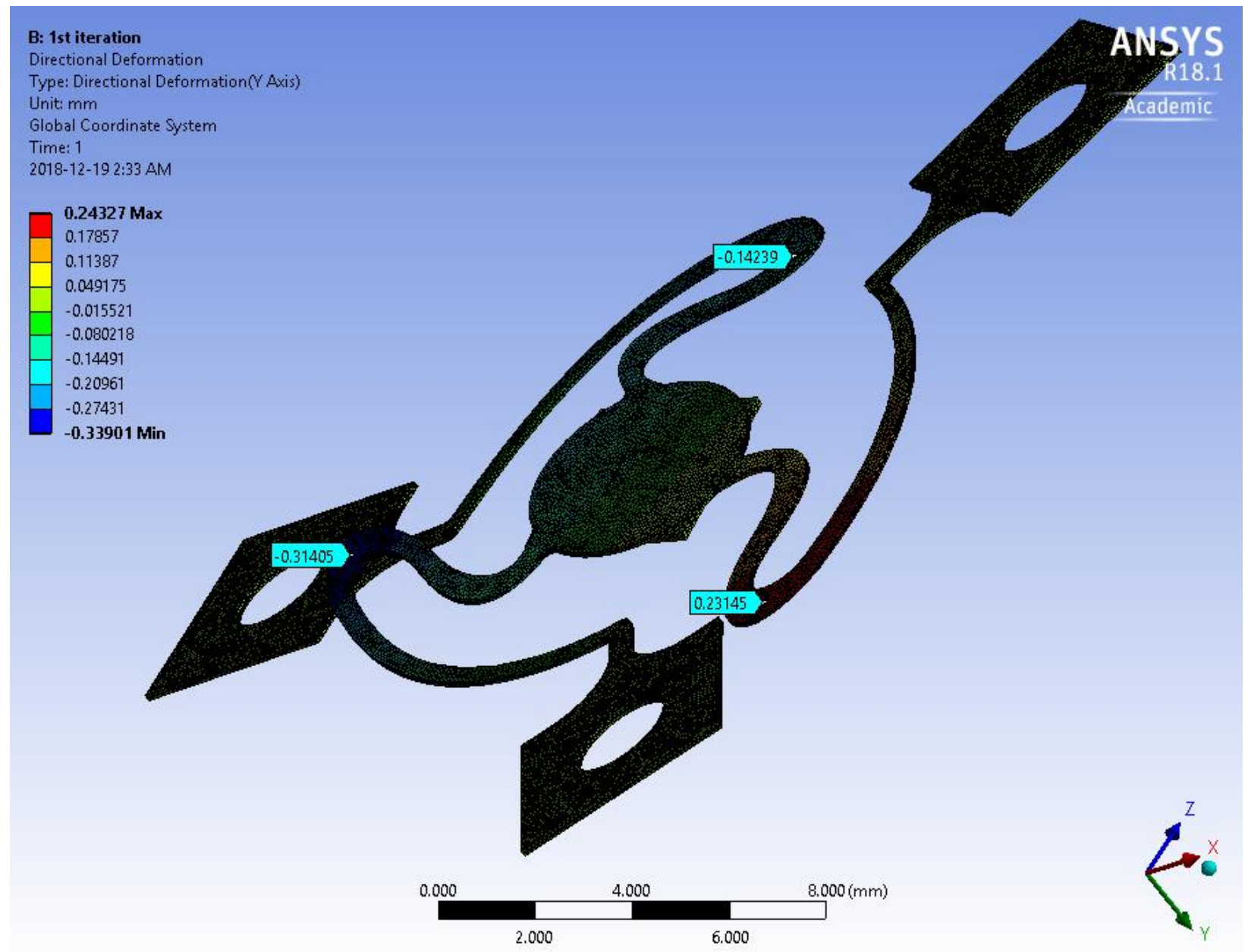

Figure 3.9 Static Structural Analysis 


\subsection{Analysis of the Results}

As there are three magnets attached at the bottom of the FPCB structure, it is difficult to deduce along which axis the mirror is rotated at the final stage. Depending upon the forces, the mirror can be rotated with respect to any three axes. These axes are formed perpendicular to the line joining the two magnets in the plane. So, in order to calculate the movement of the micromirror, laser ray is being used to deduce the laser point movement. It is assumed that a tiny triangular mirror having vertices on the three magnets is being deflected in the same manner that of the micromirror that is simulated in SolidWork. Now, the deflected mirror is modeled again in SolidWorks and the line in SolidWork is drawn replicating as a laser beam striking the mirror. In this case, the incident ray line is fixed and does not move. As now the mirror is deflected, the ray of light (line drawn in the software) may not be in the same plane on which the mirror is deflected. So, the new plane is inserted in SolidWork and the angle of incident is calculated. This new plane is perpendicular to the deflected mirror containing the previously drawn line in the plane. Depending upon the new incident angle, the ray of light (in this case a line is drawn) is reflected from the mirror. A target plane is made in SolidWorks at some distance (200 mm fixed) perpendicular to the ray of light being travelled once the mirror was not deflected. The new line is intersected at this plane and the coordinates are analyzed/ calculated with respect to the point intersected when the mirror was not deflected. This gives the movement of the laser line in SolidWorks; hence, the coordinates can be calculated in this manner. The below image depicts one such case where the laser line moved in $1^{\text {st }}$ quadrant.

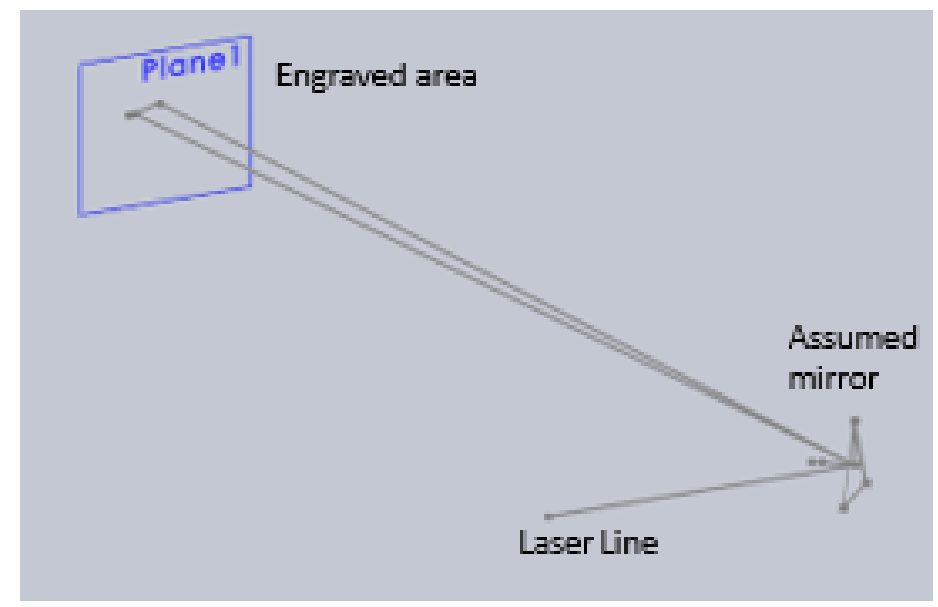

Figure 3.10 Marking the laser line point on the $200 \mathrm{~mm}$ far plane for coordinates calculation 
Table 3.6 Simulated points coordinate. *Voltage values are from calibration in order to make a square

\begin{tabular}{|c|c|c|c|c|c|c|}
\hline $\begin{array}{l}\text { Voltage } \\
\text { V1 (V) }\end{array}$ & $\begin{array}{l}\text { Voltage } \\
\text { V2 (V) }\end{array}$ & $\begin{array}{l}\text { Voltage } \\
\text { V3 (V) }\end{array}$ & $\begin{array}{l}\text { Actual X- } \\
\text { coordinate }\end{array}$ & $\begin{array}{l}\text { Actual Y- } \\
\text { coordinate }\end{array}$ & $\begin{array}{l}\text { Simulated X- } \\
\text { coordinate }\end{array}$ & $\begin{array}{c}\text { Simulated Y- } \\
\text { coordinate }\end{array}$ \\
\hline 1.57 & -1.57 & -4.37 & -5 & 5 & -4.89 & 7.01 \\
\hline 1.74 & -1.74 & -2.58 & -2.5 & 5 & \begin{tabular}{l|l}
-1.8 \\
\end{tabular} & 5.30 \\
\hline 1.81 & -1.81 & -1.00 & 0 & 5 & 1.7 & 4.60 \\
\hline 1.88 & -1.88 & 0.44 & 2.5 & 5 & 4.35 & 4.00 \\
\hline 1.88 & -1.88 & 1.68 & 5 & 5 & 6.25 & 3.15 \\
\hline 0.88 & -0.88 & 2.11 & 5 & 2.5 & 6.15 & 2.8 \\
\hline 0.85 & -0.85 & 0.87 & 2.5 & 2.5 & 4.44 & 2.89 \\
\hline 0.80 & -0.80 & -0.50 & 0 & 2.5 & 0.69 & 3.42 \\
\hline 0.69 & -0.69 & -2.06 & -2.5 & 2.5 & -3.34 & 3.38 \\
\hline 0.62 & -0.62 & -3.73 & -5 & 2.5 & -5.50 & 4.00 \\
\hline-0.36 & 0.36 & -3.17 & -5 & 0 & -5.40 & 0.89 \\
\hline-0.34 & 0.34 & -1.56 & -2.5 & 0 & -3.85 & 1.21 \\
\hline 0 & 0 & 0 & 0 & 0 & 0 & 0 \\
\hline-0.16 & 0.16 & 1.26 & 2.5 & 0 & 3.81 & 0.69 \\
\hline-0.10 & 0.10 & 2.50 & 5 & 0 & 3.75 & 0.20 \\
\hline-1.06 & 1.06 & 2.85 & 5 & -2.5 & 5.09 & -2.29 \\
\hline-1.15 & 1.15 & 1.62 & 2.5 & -2.5 & 0.71 & -3.91 \\
\hline-1.21 & 1.21 & 0.36 & 0 & -2.5 & 0.68 & -3.87 \\
\hline-1.34 & 1.34 & -1.10 & -2.5 & -2.5 & -4.72 & -1.68 \\
\hline-1.41 & 1.41 & -2.69 & -5 & -2.5 & -7.44 & -1.04 \\
\hline-2.43 & 2.43 & -2.26 & -5 & -5 & -8.07 & -3.77 \\
\hline-2.29 & 2.29 & -0.69 & -2.5 & -5 & -5.22 & -4.63 \\
\hline-2.19 & 2.19 & 0.69 & 0 & -5 & -2.28 & 2.35 \\
\hline-2.11 & 2.11 & 1.94 & 2.5 & -5 & 3.46 & 2.20 \\
\hline-1.99 & 1.99 & 3.14 & 5 & -5 & 3.98 & 3.00 \\
\hline
\end{tabular}


From the above table, the error is calculated at each point. The distance between the actual and the simulated point is calculated and then divided by the distance of the actual point from the origin in order to calculate the error. The average error comes out to be $45.2 \%$ between the actual and the simulated results.

\subsection{Determining voltage from the simulation}

In order to deduce the three independent voltages for the electromagnets, a new approach for the simulation is used which is described below. Later, these voltages are used experimentally to compare the results.

\subsubsection{Magnetic Simulation data collection}

In this approach, it is assumed that there is no interference between the magnets and the electromagnets. Each pair of magnets and the electromagnet are individually responsible for the mirror movement along their line of axis. The magnetic simulation is carried out with only one magnet and the electromagnet at different distance between magnet and the electromagnet, ranging from $2.4 \mathrm{~mm}$ to $3.6 \mathrm{~mm}$. The forces are calculated at different voltages $(-5 \mathrm{~V}$ to $+5 \mathrm{~V})$ so that the attraction and the repulsion forces data can be obtained. Below Figure XX shows the force on magnet at different distance from electromagnet varying the applied voltage to the electromagnet.

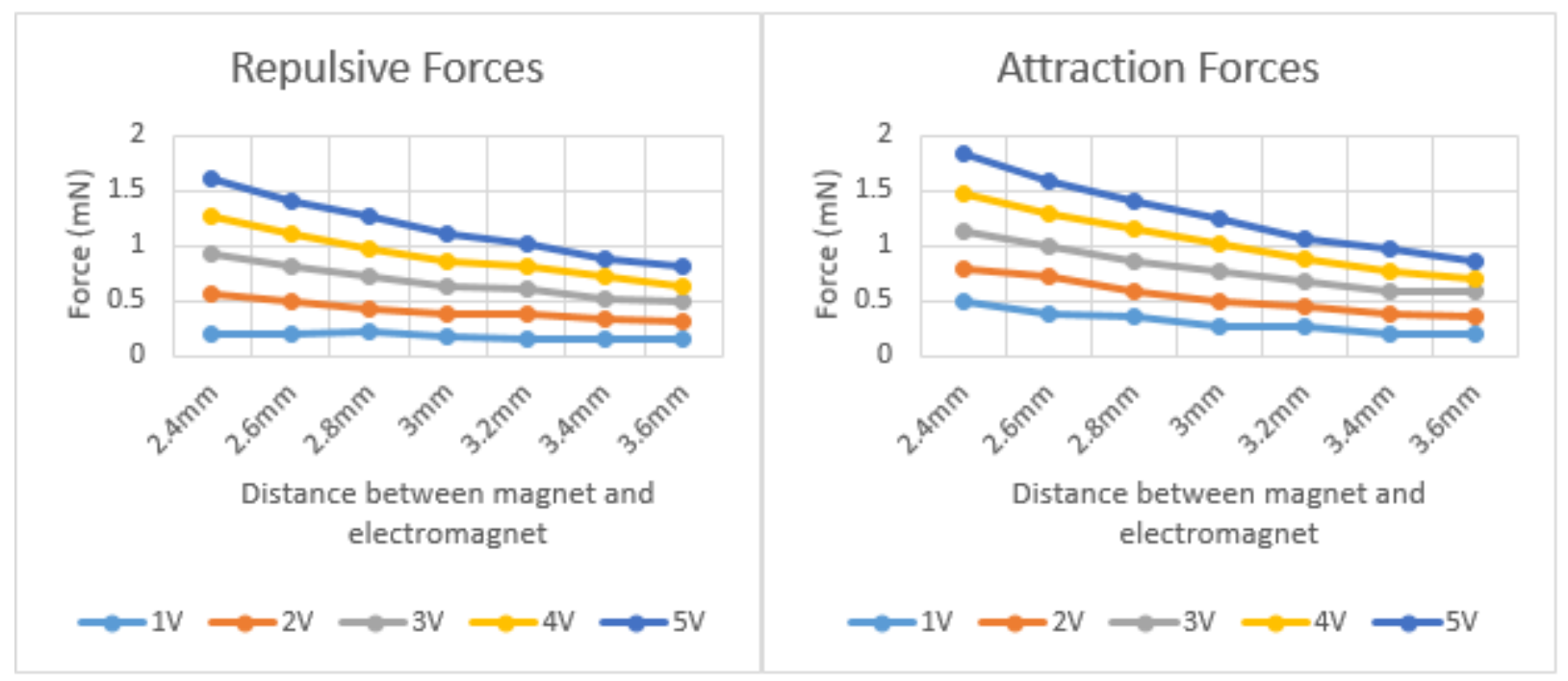

Figure 3.11 Repulsive and Attractive Force between magnet and the electromagnet 


\subsubsection{Static simulation}

Depending upon the force data calculated, few combinations of the forces ( 3 individual voltages) are applied to the static model of the FPCB micromirror. Once again, only the FPCB structure along with the silicon mirror is imported in the Ansys 18.1 static structural analysis. The magnets are omitted as they themselves are generating forces. The forces applied are selected such that there is minimal amount of translation of the mirror (i.e. 2 attractive force, 1 repulsive force or opposite). The magnitude of the forces is kept within the data collected. Using this simulation, the deflection of the micromirror corner points were noted along with the displacement of the location of the magnets in the model.

\subsubsection{Deducing the voltage from simulation}

Once the deflection of the magnets is calculated from the static structural analysis, the force values are deduced. Based on the simulated displacement of the magnets, using Figure 3.11, the corresponding voltage is calculated at the applied forces. Basic interpolation technique is used for the intermediate step values. These three independent voltages are then applied to the electromagnets to perform the experiment.

\subsubsection{Comparison of the simulated and experimental coordinates}

The experiential and simulated coordinates are compared and analysed. To generate the simulation coordinates, same technique of SolidWork line tracing described in Section 3.6 is used. In this case, the deflection of the corner of the mirror is used in the SolidWork to deduce the coordinates. These coordinates are then compared for the error calculation. In order to calculate the error, the distance between the simulated point and the engraved point is calculated and then it is divided by the distance of the engraved point from the center origin. The center/ origin is the point engraved by the laser when there is no deflection of the mirror. The average of all these errors comes out to be $48 \%$. 
Table 3.7 Comparison between the simulated and engraved points

\begin{tabular}{|c|c|c|c|c|c|c|}
\hline \multicolumn{2}{|c|}{ Simulated } & \multicolumn{2}{|c|}{ Engraved } & \multirow{2}{*}{ Distance } & \multirow{2}{*}{$\begin{array}{c}\text { Euclidian } \\
\text { distance } \\
\text { Simulated }\end{array}$} & \multirow{2}{*}{$\begin{array}{c}\text { Error } \\
\%\end{array}$} \\
\hline$X$ & Y & $X$ & Y & & & \\
\hline 3.58 & 5.54 & 3.23 & 6.35 & 0.88 & 6.596 & 13.37 \\
\hline 4.24 & 5.4 & 1.8 & 5.9 & 2.49 & 6.86 & 36.27 \\
\hline-4.06 & 5.52 & -4.1 & 1 & 4.52 & 6.85 & 65.96 \\
\hline-3.57 & -5.5 & -3.48 & -4.13 & 1.37 & 6.55 & 20.93 \\
\hline 3.7 & -5.38 & 5.5 & 1.1 & 6.72 & 6.52 & 102.99 \\
\hline
\end{tabular}

\subsection{Deviation of experimental results from simulation}

There is deviation of the experimental test results from the simulation. Firstly, there is no such software known to simulate the magnetic and the static deflection simulation as a single step analysis. Simulating the magnetic forces and the static deflection as a single step is necessary as the magnetic forces depend on the distance between the magnets and the electromagnets. Deflection in the static structure changes this distance between magnets and the electromagnets resulting the generation of the new magnetic forces on the magnets on that location. In both the above cases, the simulation is carried out as two-step process. There is significant error in the simulation which compel to generate a new method of calibration and controlling of the micromirror which is explained in the upcoming chapters.

This difference in the static results can be caused due to following reasons:

1. The plane made by the incident ray and the reflected ray should be perpendicular to the mirror surface. Moreover, this reflected beam should be normal to the target material. As the distance between the mirror and the target is $200 \mathrm{~mm}$, slight change in these parameters will result in significant change in the location of the experimental data and hence causing error.

2. The magnetic simulation also might have some factors contributing towards error. As it is assumed that the surrounding shell in which the magnetic simulation is carried out is only $150 \%$ bigger. Also, the mesh size also plays a critical role and changes the result significantly in magnetic simulations. 
3. In the simulation, the adhesive is not considered in order to make the simulation simpler. Hence, there would be the difference in the results.

4. In the simulation, it is assumed that all the magnets are in the same plane and the FPCB substrate is perfectly stretched out maintain $3 \mathrm{~mm}$ distance between magnets and electromagnets at the start of any engraving point. In actuality, the magnets might not be in the same plane.

5. The fixing of the magnet might not be in the exact same place that of the simulation. As there are no fixed physical positions in the FPCB structure to fix the magnets, this will also change the results. Also, it is impossible to ensure that the angle between the three magnets is $120^{\circ}$ while fixing magnets physically.

6. Assumption made for finding the coordinates in the SolidWorks. The replicated mirror is used to find the coordinates having vertices on the FPCB substrate where magnets are attached. This made the mirror size bigger also neglecting the deflection caused by the torsional beam in between the magnets and the mirror.

For further analysis, either a new software having a capability simulating these model as a single step or an algorithm has to be deduced from which the magnetic forces can be calculated. Also, the provision should be made in the FPCB structure to identify where exactly the magnets must be placed. This will certainly help in reducing the error.

\subsection{Resonant Frequency Mode}

The resonant frequency is calculated by using the modal analysis of the ANSYS 18.1 workbench. In this simulation, no fore is added to the body. Only the support structure is defined in the ANSYS. The natural frequency is the measure of how fast the mirror can scan the area. The natural frequency of this prototype is $36 \mathrm{~Hz}$. 


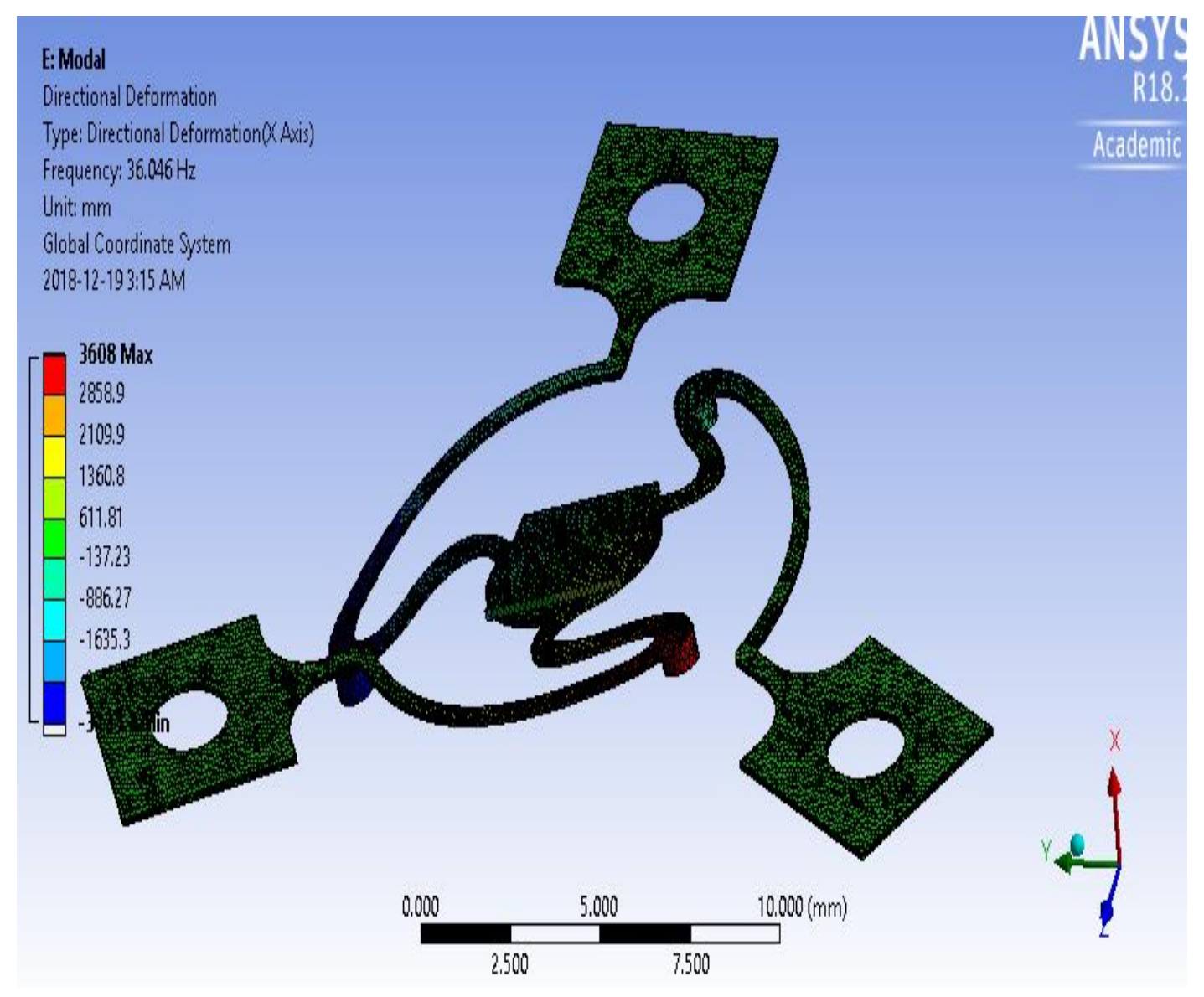

Figure 3.12 Resonant frequency testing

\subsection{Chapter Summary}

This chapter deals with the modelling and the simulation of the FPCB based micromirror. The supporting mechanical parts were designed keeping in mind the material used for mechanical support shouldn't be interfering with any magnetic field produced either by magnets or electromagnets. The simulation is carried out in two sequential steps magnetic and static structural analysis. The results were compared with the real time engraving done using current micromirror prototype. 


\section{Chapter 4: Prototyping of FPCB Micromirror}

\subsection{Fabrication of FPCB Structure}

These actuators are fabricated by the Fastline Circuits, China. A total of 5 designs was fabricated with different thicknesses, in order to have every possible factor to be considered. Following are the different design fabricated for the prototype.

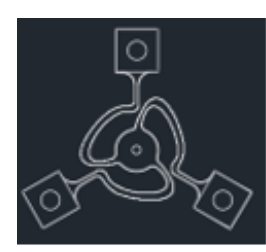

Model 1

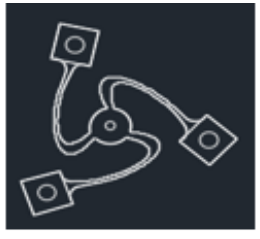

Model 2

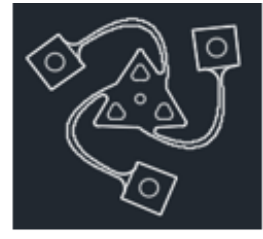

Model 3

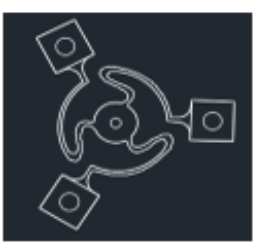

Model 4

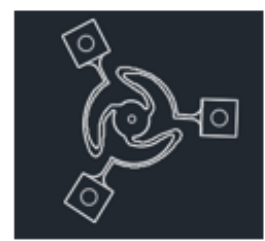

Model 5

Figure 4.1 Different design of FPCB substrate

All the designs were modeled and simulated. In order to find the most appropriate model, basic tests were done on each model. In this prototype, the model is used in quasi-static mode. The scanning area is the basis on which one of the five models is selected. Using all the model at a time, extreme endpoints were engraved to find the possible scan area of the models. Below is the table containing the scanning area of all the models. 
Table 4.1 Different prototype working area

\begin{tabular}{|c|c|}
\hline Model number & Scan area $(\mathrm{mm} * \mathrm{~mm})$ \\
\hline Model 1 & $15 * 15$ \\
\hline Model 2 & $10 * 12$ \\
\hline Model 3 & $10 * 15$ \\
\hline Model 4 & $20 * 25$ \\
\hline Model 5 & $10 * 20$ \\
\hline
\end{tabular}

Based on the scanning area of each model, Model 4 is selected and hence all the final tests were performed on this model.

The FPCB structure is fabricated by the mature commercially available process which is much cheaper than the conventional microfabrication process. As there is no circuitry involved in the design, there is no requirement to go for the conventional lithography process, hence reducing the cost of each model significantly. At present, the cost of around each model is around 20 dollars. The model fabricated is unique in design and there is a whole layer, instead of wires, of rolled annealed copper in between, which increases the cost slightly while ordering the first time. Also, as per the manufacturer quotation, the cost can be around a few dollars/ unit once it is ordered in bulk. High yield bonding of the mirror to the polymide substrate and cheap fabrication process leads to the reduction of the overall cost of the FPCB model.

\subsubsection{Fabrication of Silicon mirror}

The mirror is fabricated by dicing the silicon wafer by thin metal film. The size of the mirror used in this prototype is $4 \mathrm{~mm} * 4 \mathrm{~mm}$. As the mirror is fabricated separately, high flatness with ROC of $10 \mathrm{~m}$ can be achieved, which is more efficient when compared to the conventional MEMS mirror. Also, the reflectivity of the mirror is above $80 \%$. The micromirrors are coated with aluminium to enhance the reflectivity of the mirrors when incident by the laser beam. As per the reflectivity characteristic graph of different metals, the aluminium has greater than $90 \%$ of reflectivity at 
$445 \mathrm{~nm}$ wavelength. In terms of the cost, the aluminium coating is much cheaper when compared with other metal coating. This also reduces the overall cost of the prototype.

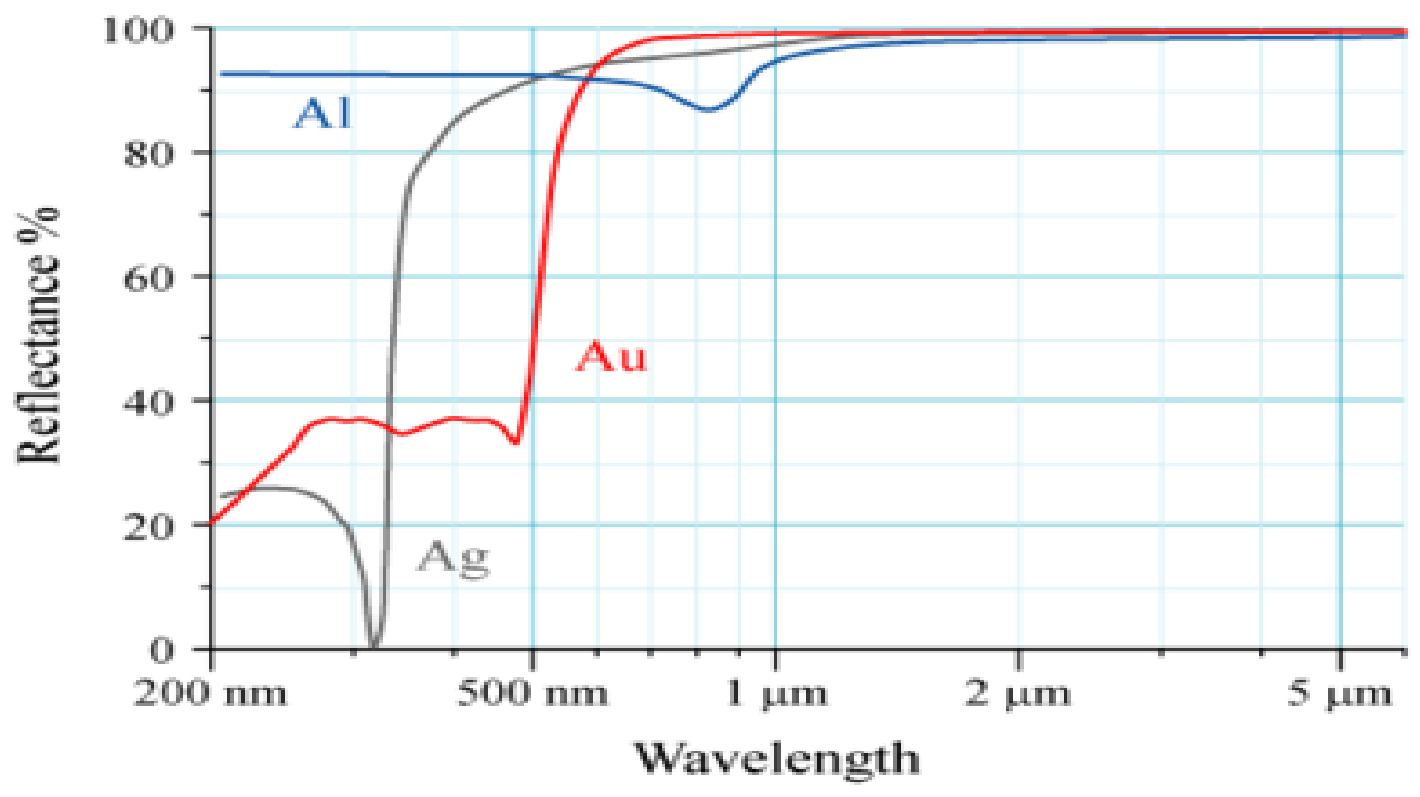

Figure 4.2 Reflectance at different wavelength [49]

\subsubsection{Cost analysis of the micromirror}

The size of the micromirror directly affects the cost of the fabrication of the mirror. However, the bigger the size of the mirror is advantageous for the laser engravers as it reduces the power loss by the laser at the cost of the speed of the engravers. Large aperture of the mirror drastically slows the movement of the mirror, hence increasing the total engraving time. $4 \mathrm{~mm} * 4 \mathrm{~mm}$ square mirrors are chosen for this prototype depending on the following cost analysis: 
Table 4.2 Cost analysis for making a silicon mirror

\begin{tabular}{|c|c|}
\hline Quantity Fabricated/ Factors & Cost Analysis \\
\hline 6-inch Silicon Wafer & $3-5$ USD \\
\hline $10004 \mathrm{~mm}^{*} 4 \mathrm{~mm}$ mirrors can be diced & $3-5$ USD \\
\hline Hourly rate of coating & 100 USD \\
\hline Total 10 hours invested to coat 1000 mirrors & $10 * 100=1000$ USD \\
\hline 10 wafers were procured & $5 * 10=50$ USD \\
\hline Grand total cost for fabricating 10000 & 10 Wafer cost + Coating cost of 1 wafer \\
& $50+1000=1050$ \\
\hline Cost of one single mirror & $1050 / 10000=0.105$ USD \\
\hline
\end{tabular}

\subsection{Laser Source}

1.6W laser with pre-installed focus from OPT laser, Europe is used in this prototype. The lens can be used to focus over a wide range. As the mirror can withstand high power, the focus can be made at a longer distance of $20 \mathrm{~cm}$. The laser used is having $445 \mathrm{~nm}$ wavelength and having the capability of TTL and analog control which can be controlled using Data Acquisition Device. The 
output beam characteristics are rectangular having a divergence angle of $2 \mathrm{mrad}$ with $5 \mathrm{~mm}$ spot diameter [50].

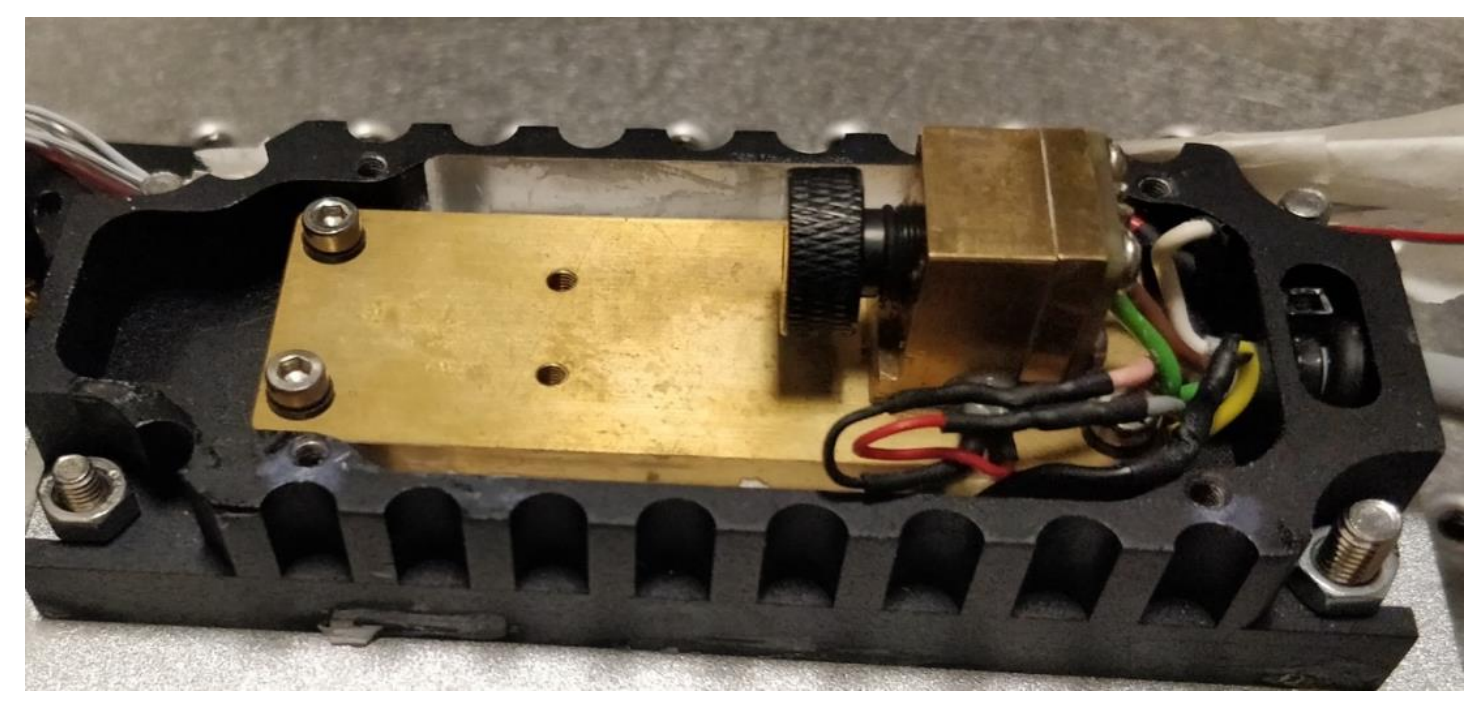

Figure 4.3 OPT Laser module with pre-installed focusing lens

\subsection{Assembly of FPCB Scanner}

FPCB scanner consists of FPCB structure and electromagnets for its actuation.

\subsubsection{Assembly of FPCB structure}

The micro-mirror is attached to the central seat of the FPCB structure using the heat resisting epoxy adhesive having curing time of around 10-12 hours at room temperature. As the substrate of FPCB, polymide, has a high strain at yield (around 3-5\% higher) than the conventional micromirror, the bonding is easier. There is less chance of damaging the FPCB structure while bonding the mirror. The mirror is pre-cleaned using the mirror cleaning liquid. The working temperature of the epoxy is around $180^{\circ} \mathrm{C}$ which is much higher than the heat generated by the incident laser beam. Once the micro-mirror is fixed, then it is visually inspected for the accuracy and the strength of the bonding. After the mirror, three small magnets are glued using epoxy at the bottom of the central seat, on the torsional beam. The magnets are installed in such a manner that the distance of the magnet from the center is maximum to generate enough torque to rotate the mirror. The distance 
is calculated based on the simulation result and effort is made to replicate the simulated structure to achieve the similar results of simulations. A second important factor to consider while fixing the magnets is that the three magnets are facing the same poles in the same direction subtending $120^{\circ}$ in between them. This is to ensure that there is symmetry in the fixed model while generating torques in the center. A gap of $3 \mathrm{~mm}$ must be maintained between the magnets and the electromagnets in order to avoid the dip in of the magnets. As experimentally proven and simulated, at around $1.7 \mathrm{~mm}$, the magnets start attracting to the electromagnetic core even if the magnetic force generated by the electromagnet tries to repel them. The entire assembly is left untouched for 24 hours to cure the applied epoxy.

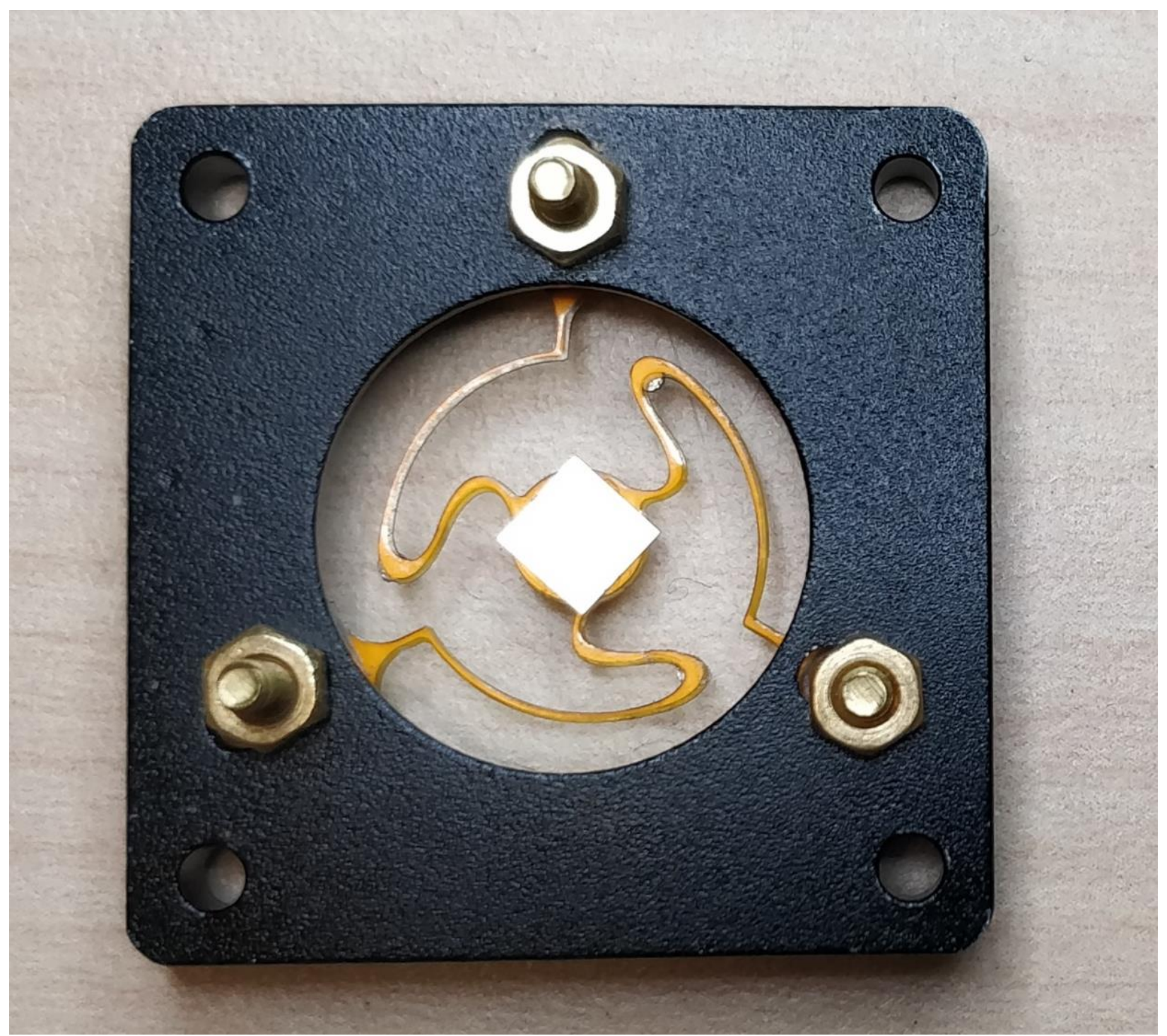

Figure 4.4 FPCB Micromirror 


\subsubsection{Scanner Setup}

Once the FPCB structure is assembled and cured for 24 hours at room temperature, the structure is screwed to the fabricated aluminium seat using M2 screws at the supporting pads of the structure as shown in the following figure. While fixing the FPCB structure to the mechanical aluminium seat important steps should be taken care of:

1. The FPCB structure should be stretched a little so that there is no slack in the model.

2. The magnets installed on the structure should be parallel to the surface of the FPC substrate and all the magnets should be in one plane.

3. While tightening of the screw at the supporting pads, it should be ensured that the FPCB structure is not twisted. The following idea can be used to minimize the effect of the tightening of the screw on the model. The screw is added in between the FPCB and the mechanical part to make the model perfectly straight.

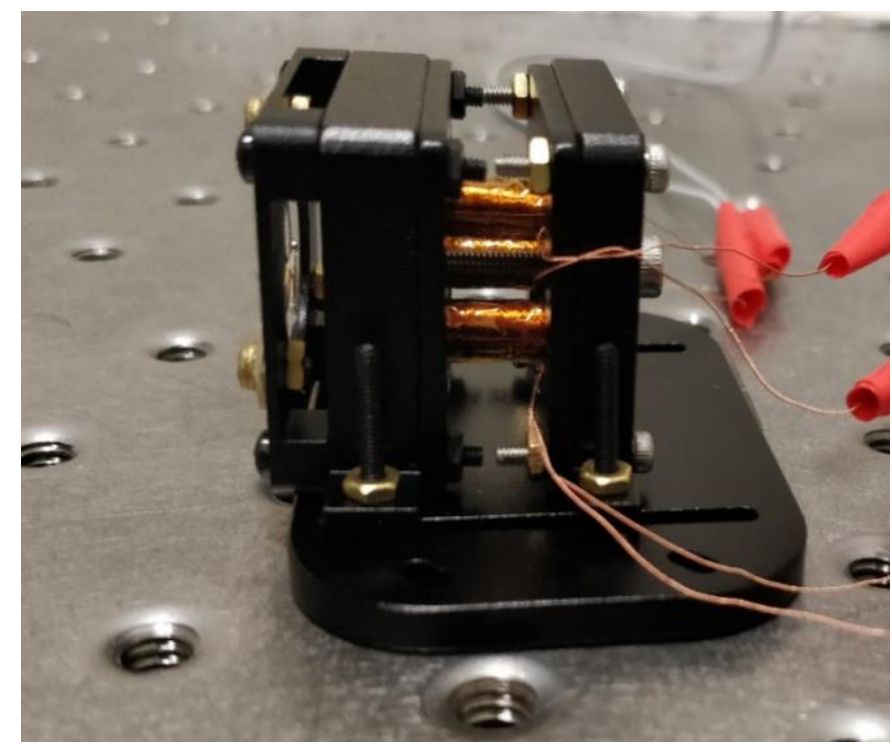

Figure 4.5 FPCB based Micromirror assembly

\subsubsection{Actuator Setup}

The FPCB structure with the aluminium metal seat is then placed over the electromagnets with 3 $\mathrm{mm}$ gap between each magnet and electromagnet. As per simulated value $1.7 \mathrm{~mm}$, distance between magnet and an electromagnet is needed to avoid the dip-in effect. Dipping in effect can be described as the magnet is pulled towards the electromagnet even when the electromagnet is 
actuated. The force of attraction between the electromagnet core and the magnet overcomes the effect of electromagnetic repulsive force. In this condition, at this distance the magnet is pulled in and physically sticks to the electromagnet core. At most care to prevent physical stickness of electromagnet core with the magnet is always taken while doing tests, calibration, and final engraving. The three electromagnets are placed parallel to each other and $3 \mathrm{~mm}$ apart to the magnets.

\subsubsection{Alignment of the FPCB Scanner}

The whole scanning model is placed in such a way that the micromirror is vertical with respect to the base so that the laser beam can incident on the mirror at $45^{\circ}$. However, in order to ensure that the laser beam strikes at the center of the mirror, proper arrangements are made. The design was made in such a way that there is a provision to move the scanner in all three directions to align it with the incident beam. The scanner was installed on the base plate and then mounted on the engraving model. This base plate has a groove capable of moving the scanner in plane left and right. The corresponding groove was made on the engraving base plate as well to accommodate the movement. Also, to move the scanner vertically upwards and downwards with respect to incident laser beam, thin aluminium plates were fabricated to ensure the exact height of that of the laser beam can be reached.

\subsubsection{Assembly of FPCB Engraver System}

The laser diode is mounted on the base of the metal plate and the scanner is aligned using the base plate and the thin aluminium plates in such a way that the incident beam strikes the mirror at $45^{\circ}$. The care to be taken when aligning that the mirror is completely vertical to the laser incident beam and $45^{\circ}$, as the minor misalignment can cause a huge error. The mirror has 2-dimensional movement and misalignment can cause loss of power and non-accurate engraving patterns. The overall size of the engraving system is about $17 \mathrm{~cm} * 10 \mathrm{~cm} * 7 \mathrm{~cm}$. The footprint size of the FPCB scanner is about $28 \mathrm{~mm} * 28 \mathrm{~mm} * 28 \mathrm{~mm}$ only. The size of this 2 -dimensional FPCB scanner is much smaller than the conventional MEMS scanner currently present in the market. Also, the size of the whole system can further be reduced by replacing it with small dimension laser as the major space occupied is by laser itself. 


\subsection{Controlling of the Micromirror: LabView Code}

The whole engraving system is now set up and the micromirror can be actuated by energizing the electromagnet. As the current is provided in the electromagnet, depending upon the direction of the current, the polarity of the electromagnet will be generated causing the magnets to attract or repel. This will cause the mirror to rotate in the desired orientation. The control of the current to the electromagnets is done by using Data Acquisition System (DAQ) by National Instrument (NI) (Model number: NI USB 6343). For the control of the three electromagnets and the ON/ OFF laser module 4 channels DAQ is required that can run simultaneously, hence controlling the whole engraving system can be done. The controlling of DAQ can easily be integrated into LABVIEW programming (Laboratory Virtual Instrument Engineering Workbench) [51]. This workbench has predefined code blocks to use and it is a graphical coding which makes the code easier to understand for the users. This coding is relatively more user-friendly when compared with another programming language. For controlling the DAQ system LABVIEW provides full support via DAQmx software integrated within it. Also, the benefit of using LABVIEW to control the DAQ is that the LABVIEW is well-developed software that is compatible with other microprocessors and other embedded platforms.

The main aim of using the LABVIEW is to control the three-electromagnet current and the laser modulation. The series of the voltages for the electromagnet and the laser has to be supplied to the electromagnets and the laser simultaneously from the DAQ. Here the timing of supplying of the voltages is critical as vector engraving is to be done. The laser modulation timing should be done exactly when the mirror is actuated and reached the final position for steering the laser beam to the desired location. Also, the mirror settling time also plays a major role for the timing of the laser on and off. The detailed code is described in the next section. 


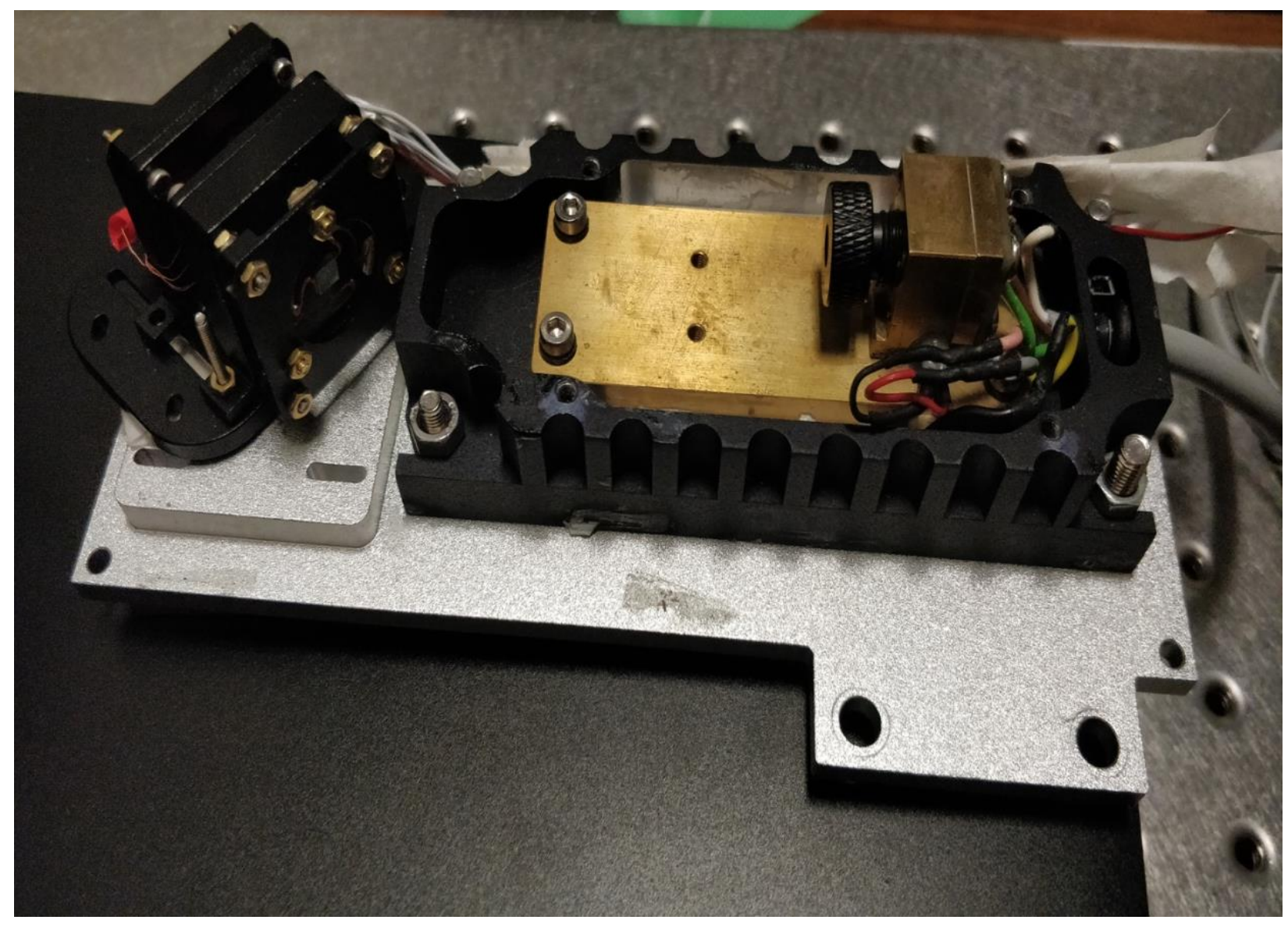

Figure 4.6 Assembly of the Laser Marking/ Engraving system

\subsubsection{LABVIEW Program}

This section contains the entire coding that is made in the LABVIEW to control the DAQ device. The objective is to input voltages of the desired points for the 3 electromagnets and the laser modulating voltages. Depending upon the electromagnet resistance the current is driven in each electromagnet. In order to coordinate between multiple DAQ channels, the code is generated in the LABVIEW. The predefined code blocks are not being used as they have limited functions for the desired result. For better control of the current in the electromagnets, few DAQ commands are rearranged to solve the purpose.

Following is the full command that is used to control the entire engraving system. 


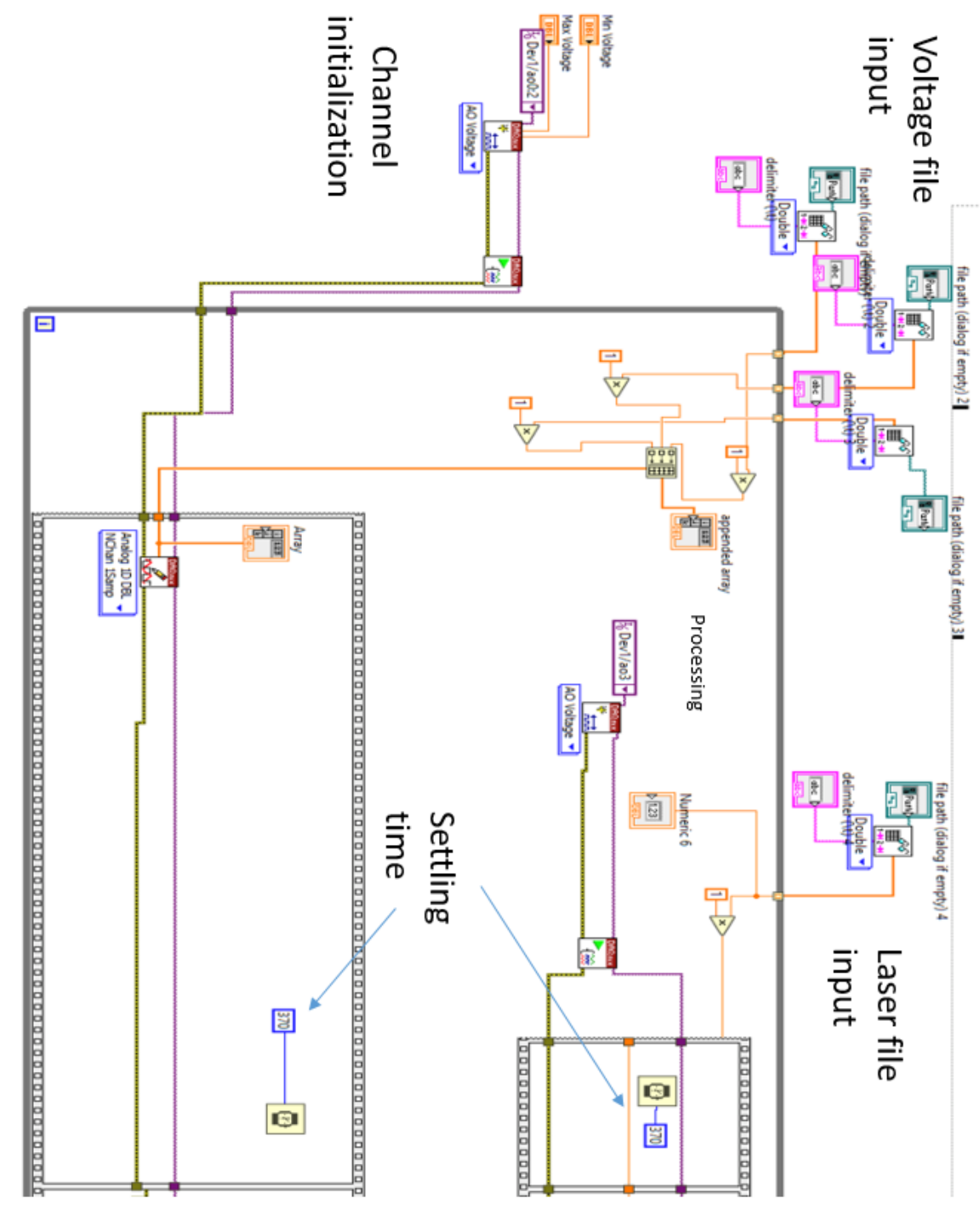

Figure 4.7 LABVIEW code for the micromirror settling time 


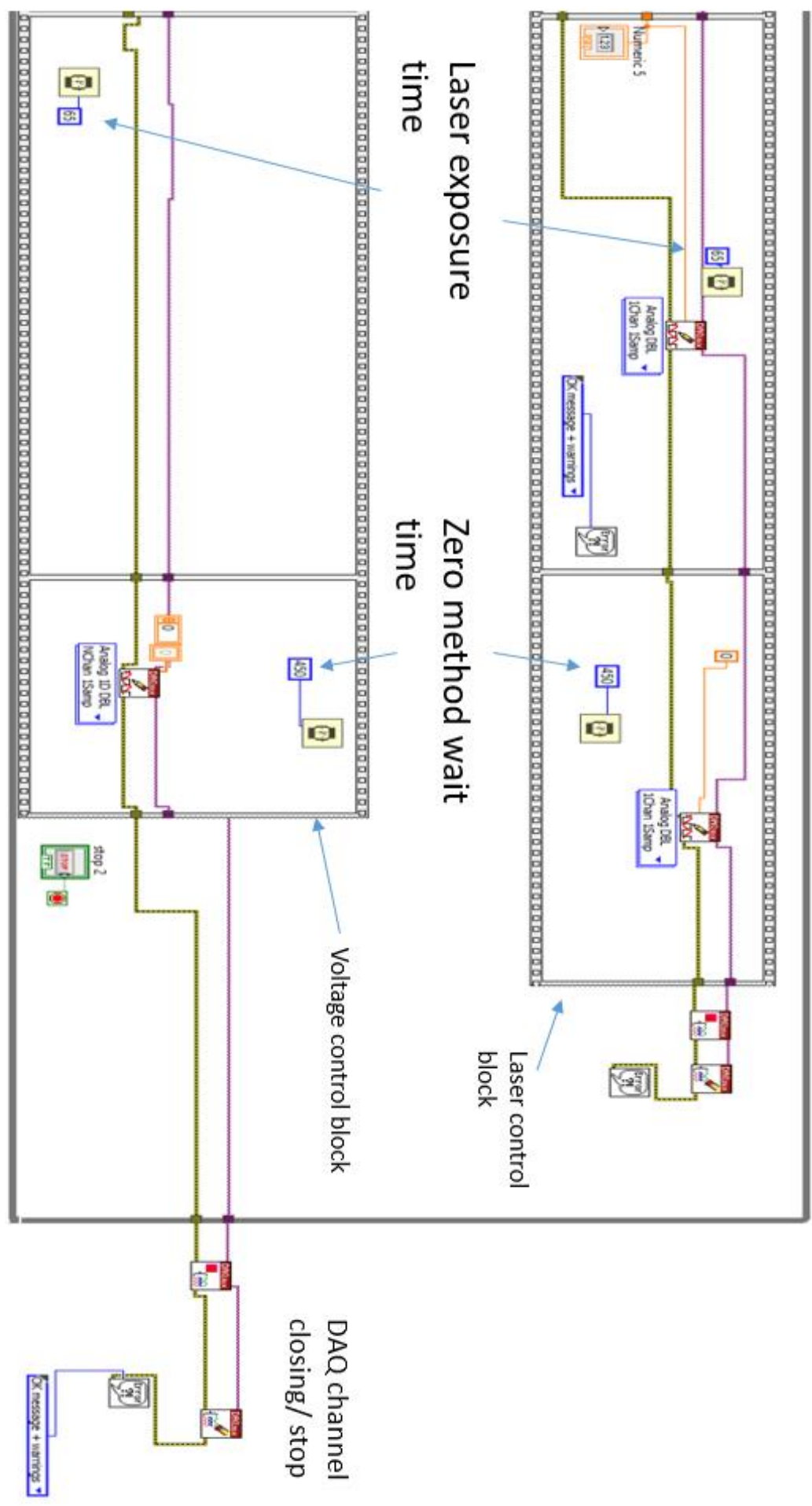

Figure 4.8 Zero method code for the LABVIEW software 
The above program is utilized in engraving different patterns on the target surfaces. The resistance of the electromagnet is $72.5 \mathrm{ohms}$. The current for each electromagnet can be calculated making use of this resistance of the electromagnet. The different combination of the three voltages is to be calculated for actuating the mirror at the desired location. The 3 voltage files are made as column values in .txt format and fed into the LABVIEW program. A separate file for the laser modulation in a similar manner is also provided in the software as an input. The whole program works with a single time clock to make the whole output in a synchronous fashion.

\subsubsection{Program Analysis}

The LABVIEW program is enclosed in the while loop to run the program continuously. Inside the while loop, the program is mainly divided into two sections. One section is responsible for the mirror actuation as a sequence structure. In this section, the sequence starts with inputting the three voltages as a single input and utilizing the three channels of the DAQ. As soon as the voltages are applied from DAQ to the electromagnets, the force is exerted to the magnets and the mirror is rotated to the desired position. On the other sequence, the laser modulated value is fed. However, the voltage is fed to the laser only after the mirror settle to the desired particular position (settling time: 370 milliseconds explained in the next section). The laser is kept on for 65 milliseconds and then the laser is set off. After this stage zero voltage is given to all the electromagnet and the next set of voltages after the mirror comes back to the initial stage. This is known as ZERO method which is explained later in the section. All these parameters are analyzed in detail in later sections.।

\subsection{Setup and Testing}

After making the code for the control of actuating, basic tests were done in order to compare with the simulated result. In this section, the characteristics of the micromirror are analyzed. It includes some parametric tests like settling time, natural frequency and the response time of the mirror. As DAQ is used to actuate the electromagnets and laser, there is one drawback to deal with. National Instrument DAQ can generate voltage, however, it cannot generate enough current to actuate the electromagnet. In order to actuate the electromagnet, current amplifier (electromagnet driver) is used to ensure that enough current can be generated for the electromagnet. The driver was designed and fabricated by a colleague Hui Zhou. The driver has 3 input and 3 output channels. The electromagnet is driven by DAQ via these channels. 


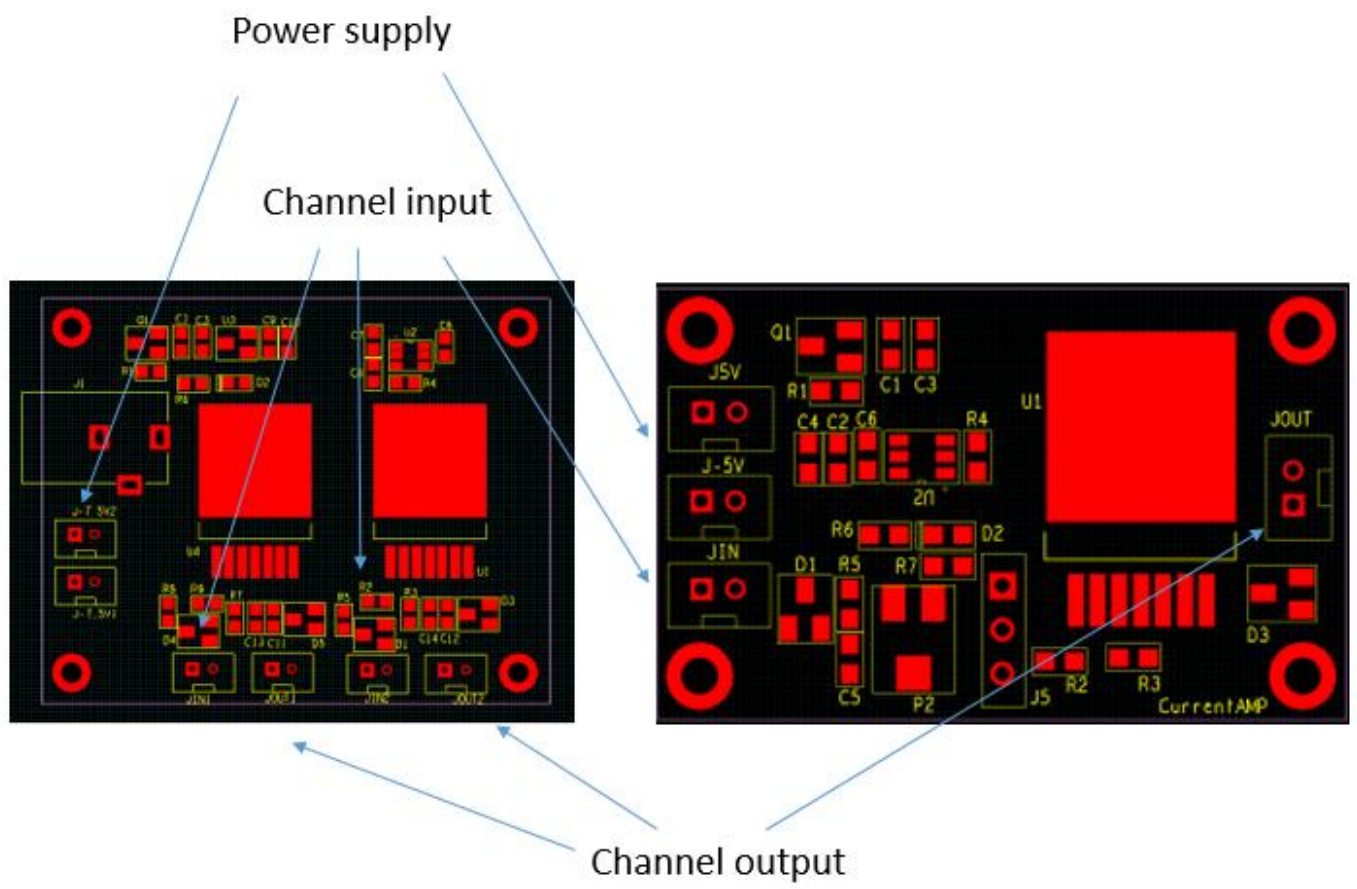

Figure 4.9 Electromagnet drivers

The channels can generate enough current for the input signal to drive the electromagnet. Some of the testing conditions are described below:

1. Based on the basic parametric tests and simulations, one of the models is finalized for the prototype to be carried out.

2. All the tests were done after analyzing the problems and implementing in the code. Zero method is proposed which is described later.

3. Assuming that the FPCB structure fixed is perfectly straight and all the magnets are in the same plane when doing experiments.

4. All the electromagnets are identical having the same characteristics.

\subsubsection{Static Testing of the Micromirror}

In this testing, the mirror is actuated by providing specific voltages to the electromagnet via DAQ. As this is a 2-dimensional micromirror, it will steer the incident laser beam in every quadrant of the workpiece (leather/ material to be engraved). In this prototype, there are three magnets aligned at $120^{\circ}$ apart. The line joining these magnets will serve as a rotational axis of the micromirror. So, there are three axes on which the micromirror can be rotated in order to reach the desired point in the space. Therefore, it is difficult to calculate the resultant angle on which the mirror is rotated and reached the final stage. However, it is relatively easy to distinguish/ measure the point 
engraved on the leather. Hence, after supplying the voltage, the engraved point coordinates are measured. All the engraving done is at $200 \mathrm{~mm}$ away target material from the micromirror scanner. To start off with the experiment, the scanner is aligned with the laser beam at low power. The leather is placed, and the origin is marked on the leather with the pencil. This point is considered as a center/ origin for the rest points. All the engraved points coordinates are calculated from this point. In this case, to simplify the combination of the current values to the electromagnet, out of three current values, two values are equal, but the direction of the current is reversed. The third one is independent of the other two. A total of 441 points with different voltages was input and below are just a few points for the reference.

Table 4.3 Experimentally engraved points coordinates

\begin{tabular}{|c|c|c|c|c|}
\hline Voltage V1 (V) & Voltage V2 (V) & Voltage V3 (V) & X-coordinate & Y-coordinate \\
\hline 4 & -4 & -5 & -10 & -8.8 \\
\hline 5 & -5 & -4 & -9.11 & 11.37 \\
\hline 5 & -5 & -3 & -7.82 & -11.61 \\
\hline 5 & -5 & 2 & -6.32 & -11.2 \\
\hline 5 & -5 & -1 & -4.63 & -11.9 \\
\hline 5 & -5 & 0 & -3.08 & -12.12 \\
\hline 5 & -5 & 1 & -1.10 & -12.22 \\
\hline 5 & -5 & 2 & 0.95 & -12.58 \\
\hline 5 & -5 & 3 & 3.13 & -12.84 \\
\hline
\end{tabular}

To calculate the relation between the current and to the point on leather, many points in the scanning area are engraved and the coordinates are noted with the applied current combinations to the three electromagnets. After that, using a software Table Curve 3D, the curve is tried to fit between voltage and X-Y coordinate of the point. A detailed method is described in the next chapters for engraving any desired pattern. 


\subsubsection{Resonant Frequency Test for the Micromirror}

Resonant/ Natural frequency test is critical for the micromirror to analyze how quickly the micromirror can move at resonance. At resonance, for the specific current value, the mirror oscillates with the highest deflection hence providing the largest angle. In this prototype, the mirror has the capability to rotate in two dimensions. However, due to the symmetry in the model, all the natural frequencies would be equal in both the axes. For this testing, the current drivers are replaced, and the sinusoidal wave is provided to the electromagnet. In order to calculate the frequency, the current is kept constant and the frequency is varied. To ensure that there is a pure rotation of the micromirror and there is no translation motion involved, the electric current values are decided such that the two of the electric current values are half and the direction is reversed in comparison to the third one. By doing this, the mirror is balanced, and no force is generated which is responsible for translational motion. There is assumed to be a pure rotation of the micromirror. Also, as it is a 2-dimensional mirror when there is a phase change in the voltages forming couple the laser beam will scan the area in an elliptical shape. The natural frequency of the micromirror comes out to be $41 \mathrm{~Hz}$ for an applied voltage of $5 \mathrm{~V}$ to one and 2.5 volts to another two opposite directional currents to the electromagnets.

\subsubsection{Settling Time of the Micromirror}

This tests for the micromirror is done to quantify how quickly the micromirror can response to the actuation mechanism. It also gives the idea of the stability of the micromirror by determining the settling time of the mirror. Settling time can be described as the time taken by the mirror to settle down after taking one step. For this model, this data is calculated at $+/-5 \%$ of the mean value for the maximum step i.e. the maximum amount of laser point moves. It helps in predicting the realtime performance of the mirror. In order to calculate this characteristic of the micromirror, the high-power laser is replaced by the visible laser and the reflected laser beam from the mirror is projected on the Positioning Sensing Device (PSD) (ON-TRAK 2D PSD) coupled with oscilloscope to visualize the real-time data fluctuation on the PSD. The PSD used is combined with the signal amplifier before processing it to the oscilloscope. This also helps in reducing the noise in the signal. In this test as well, the connections were made to the electromagnet in the similar fashion as in the previous case however in place of sinusoidal wave a step wave is applied. The settling time of the micromirror comes out to be $368 \mathrm{msec}$. 


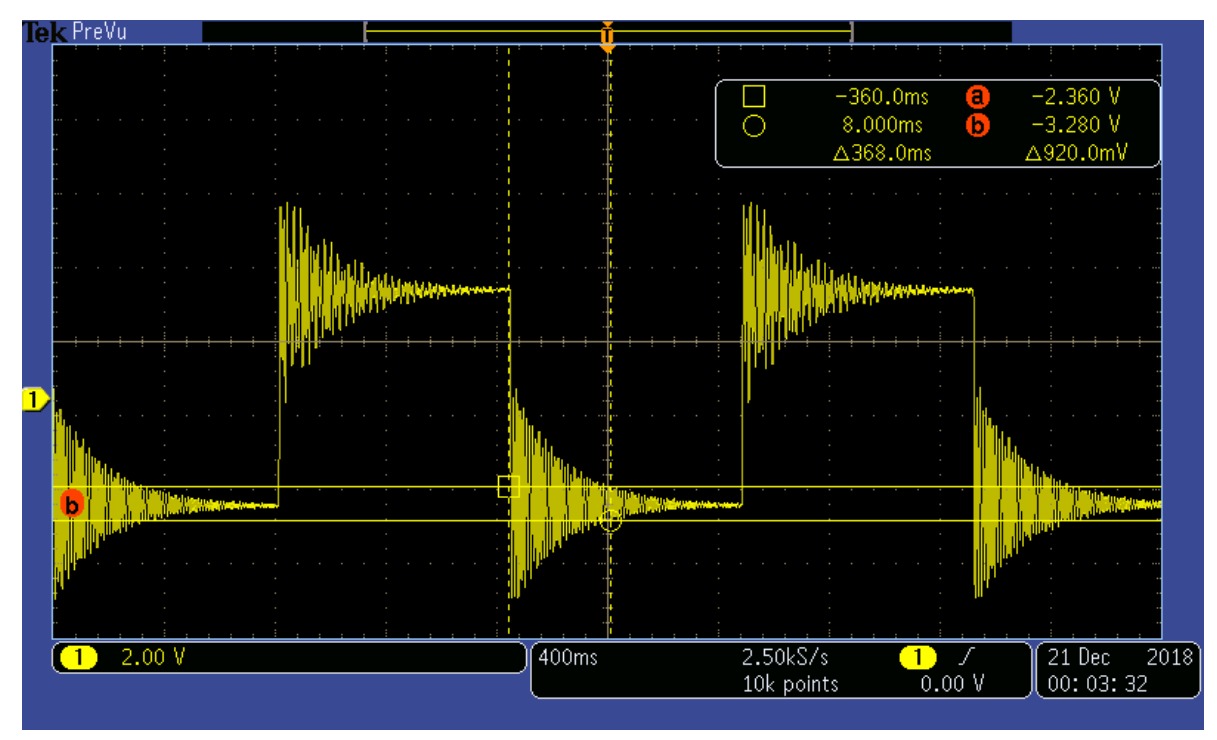

Figure 4.10 Settling time for the micromirror

\subsection{Power Analysis for the Current Prototype}

Power plays a critical role in designing any engraving system. However, the mirror which is used to reflect the laser beam should also be capable of withstanding that much amount of power. As the substrate material of the prototype is polymide, the prototype has a maximum power limitation of a maximum of $0.97 \mathrm{~W}(970 \mathrm{~mW})$. Increasing above this amount of power, the prototype can engrave, however, there is a significant amount of drifting that can be observed while engraving with a higher power. This can be due to the polymide material, which starts wrapping because of high power. Also, the reflectivity of the mirror is also a decisive factor that how much power is lost in reflectivity as heat causing the heating of the substrate material.

\subsubsection{Power Testing Setup}

In order to test the power of the laser source and to calculate how much power is lost as heat from the mirror, it is necessary to do the power testing of the whole engraving system. First, the laser source itself is tested that how much power is being generated by the laser diode source and then secondly how much power is being reflected by the micromirror. In order to calculate power, the standard Power meter is used which is capable of measuring $2000 \mathrm{~mW}$ at 3 decimal places effectively. 


\subsubsection{Testing of the Laser Diode Source}

The setup is made with only the laser mounted on the base plate and it is directly focussed on the power meter. The power of the laser beam is measured at different locations in order to test the efficiency of the laser source. See table 4.6 for the power values at a different location with modulation of the laser on the next page below.

\subsubsection{Testing the Reflectivity of the Mirror}

Once the diode laser source power is measured the mirror is installed on the base plate and then again, the power is measured. This time the laser beam is reflected by the mirror at $90^{\circ}$ from the initial laser source striking at $45^{\circ}$ to the mirror. The power is calculated at the same distance calculated from the laser source in order to calculate the loss of the power from the mirror assuming no other losses. This loss of power causes heat on the micromirror structure and hence limiting the maximum withstanding capability of the prototype. The reflectivity of the mirror is calculated $76.6 \%$ for the current mirrors.

\subsection{Quantitative Analysis of Power}

Below Table 4.5 shows the reflectivity performance for the mirror used in this prototype. Power is calculated from the laser diode before and after reflection from the mirror. The average calculated reflectivity is $76.6 \%$.

Table 4.4 Power of the laser before and after striking the micromirror

\begin{tabular}{|c|c|c|}
\hline Modulation Voltage & Power before striking mirror & Power after mirror at 200mm \\
\hline $1 \mathrm{~V}$ & $0.128 \mathrm{~W}$ & $0.094 \mathrm{~W}$ \\
\hline $2 \mathrm{~V}$ & $0.564 \mathrm{~W}$ & $0.439 \mathrm{~W}$ \\
\hline $3 \mathrm{~V}$ & $0.972 \mathrm{~W}$ & $0.719 \mathrm{~W}$ \\
\hline $4 \mathrm{~V}$ & $1.356 \mathrm{~W}$ & $1.085 \mathrm{~W}$ \\
\hline $5 \mathrm{~V}$ & 1.672 & $1.287 \mathrm{~W}$ \\
\hline
\end{tabular}




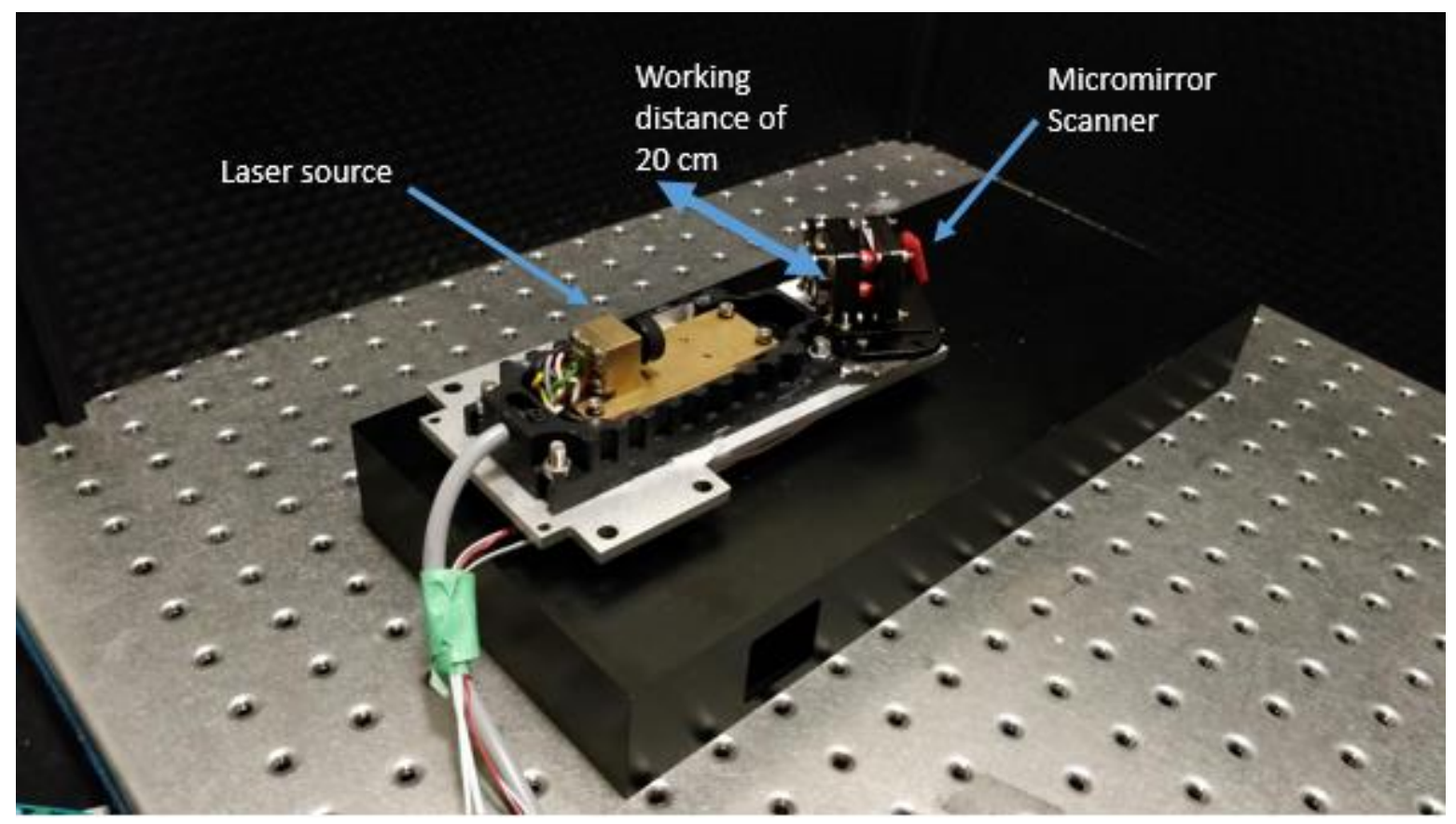

Figure 4.11 Final Prototype of Laser Marking/ Engraving System

The above photograph depicts the prototype of the laser marking/ engraving system with the working distance of $20 \mathrm{~cm}$.

\subsection{Chapter Summary}

In this chapter, the salient features of the micromirror is characterised by performing basic experiments. This chapter deals with the prototyping of the micromirror scanner and its application for the laser marking/ engraving system. The cost analysis is done on fabricating the current prototype. It also describes about the assembly and the setup of the micromirror scanner which is critical for laser marking system performance. The complete system prototype is assembled for the final tests. 


\section{Chapter 5 Control Method for Laser Marking/ Engraving System and Method of overcoming Drifting}

The scanner in this system works on the principle of magnetic force of attraction and repulsion. The laser beam is steered by the movement of the micromirror. To move the micromirror, magnets are attached to the mirror and these magnets experience the force of attraction and repulsion by the electromagnet placed beneath them. The electromagnet either attracts or repel the magnets depending on the direction of the applied current, which can be controlled by the driving voltage to that electromagnet. To steer the laser beam towards any desired point, the driving voltage is needed that to be applied to the corresponding electromagnet, in this case combination of three voltages. Random 441 points are engraved, by two voltages, making a couple (ranging from $-5 \mathrm{~V}$ to $+5 \mathrm{~V}$ ) with the third to be ranging from $-5 \mathrm{~V}$ to $+5 \mathrm{~V}$. The coordinates of the engraved point are measured with the reference point, considering the reference point, to be the one when the voltage applied to all the electromagnet to be zero. Below are a few entries from that table (Table5.1) in the reference.

Table 5.1 Few entries from the calibration data

\begin{tabular}{|c|c|c|c|c|}
\hline Voltage (V1) & Voltage (V2) & Voltage (V3) & X-coordinate & Y-coordinate \\
\hline-5 & 5 & 3 & -9.40 & -6.39 \\
\hline-5 & 5 & 2 & -8.80 & -4.04 \\
\hline-5 & 5 & 1 & -8.05 & -1.63 \\
\hline-5 & 5 & 0 & -7.31 & 0.767 \\
\hline-5 & 5 & -1 & -6.56 & 3.069 \\
\hline-5 & 5 & -2 & -5.72 & 5.423 \\
\hline-5 & 5 & -3 & -4.87 & 7.827 \\
\hline-5 & 5 & -4 & -3.88 & 10.23 \\
\hline-5 & 5 & -5 & -2.88 & 12.63 \\
\hline-4.5 & 4.5 & -5 & -2.18 & 12.68 \\
\hline-4.5 & 4.5 & -4 & -3.03 & 10.64 \\
\hline-4.5 & 4.5 & -3 & -3.93 & 8.44 \\
\hline
\end{tabular}


From this table, to reach the coordinates given in the table, the combination of the voltages (i.e. V1, V2 and V3) should be applied to the respective electromagnets. This will cause the mirror to deflect in that orientation so that the laser can be reflected to the location desired by the user. This is done to deduce the voltage combinations of the space surrounding the engraved points so that the laser can be steered to any given point in the calibrated region. In order to calculate the combination of the voltages to reach any coordinate, three curves are generated in MATLAB having the coordinates of the point as $\mathrm{X}$ and $\mathrm{Y}$ axis respectively, with one of the electromagnet voltages (V1, V2, V3 one at a time) as the $\mathrm{Z}$ axis. Below is one of the surface fit curves. Similarly, other two graphs can be generated as well.

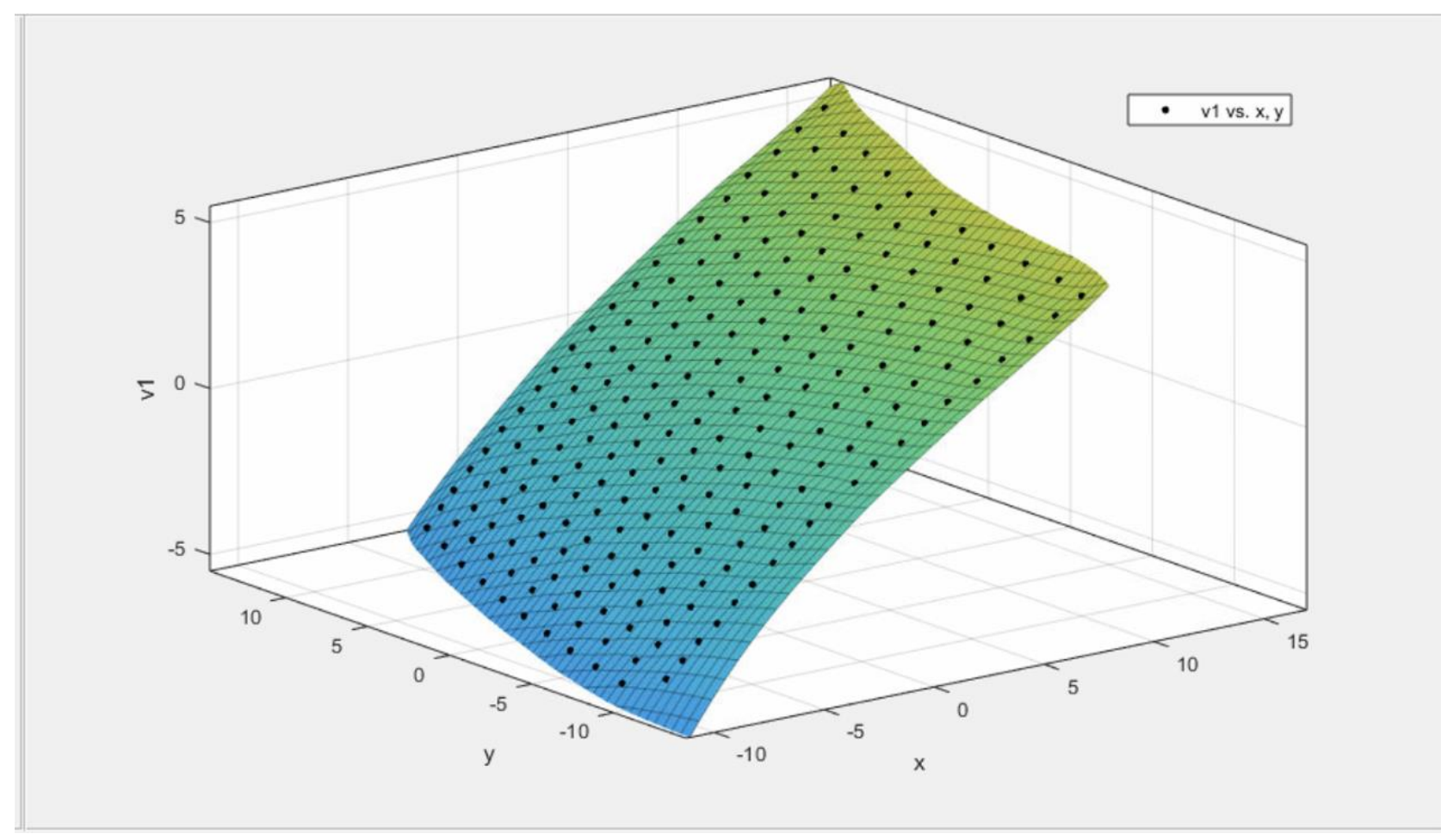

Figure 5.1 MATLAB surface curve

These three surface curves can be used to calculate the voltage combinations to reach any given coordinate in the calibrated region. However, it is to be noted that these coordinates in the table are calculated when the mirror deflects from its initial position, when it is not experiencing any force from electromagnets. Deflected magnets would experience changed forces from that when they are at their initial positions. In this case, a series of combination of voltages are applied to engrave the target material, the second iterative force values would differ from the calibrated value due to the change in distance between the magnets and electromagnets. By using this basic idea, 
the combination of the voltages to steer the reflected beam to the desired location can be calculated which can be used to engrave any desired pattern. However, the precision of the pattern would depend upon the accuracy of the curve and the interpolation technique used. In general, FPCB micromirror has an advantageous edge over other conventional MEMS mirror due to its high strain at yield, lightweight and low cost especially in quasi-static mode. However, in this current prototype, drifting and creep properties associated with this quasi-static polymide FPCB substrate is visible. There are two major problems that are identified and addressed for the accurate engraving pattern for this prototype. Working on the above said basic idea, following problems were encountered. The proposed solution could be used in order to minimize their effect and better control of the micromirror.

\subsection{Drifting problem description}

From the previous basic testing, it is evident that the drifting is because of the visco-elastic behavior of the FPCB polyimide substrate and have a significant effect when working at quasistatic mode of the micromirror [52][53]. In this prototype, the principle of actuation is based on the force of attraction and repulsion of magnets and electromagnets in which the distance between them plays a major role. The force values of the magnets will change even at the same current combination when this distance is changed, as the magnetic field will become weaker as the distance increases and vice-a-versa. At the time of calibration, the forces are calculated keeping that the magnets are always at an initial distance. However, when we continuously input the series of voltage combinations, the distance is different from the second point onwards. The magnets are no longer at $3 \mathrm{~mm}$ from the electromagnet. This changes the force values acting on the mirror hence the mirror can't be deflected/ rotated at the desired orientation. Due to this engraved image is highly distorted in shape and size. Below is the image of the engraved material depicting this specific problem only. Here a series of combination voltages are applied to the electromagnet which causes the micromirror to deflect the incident laser beam. The points engraved is highly distorted. 


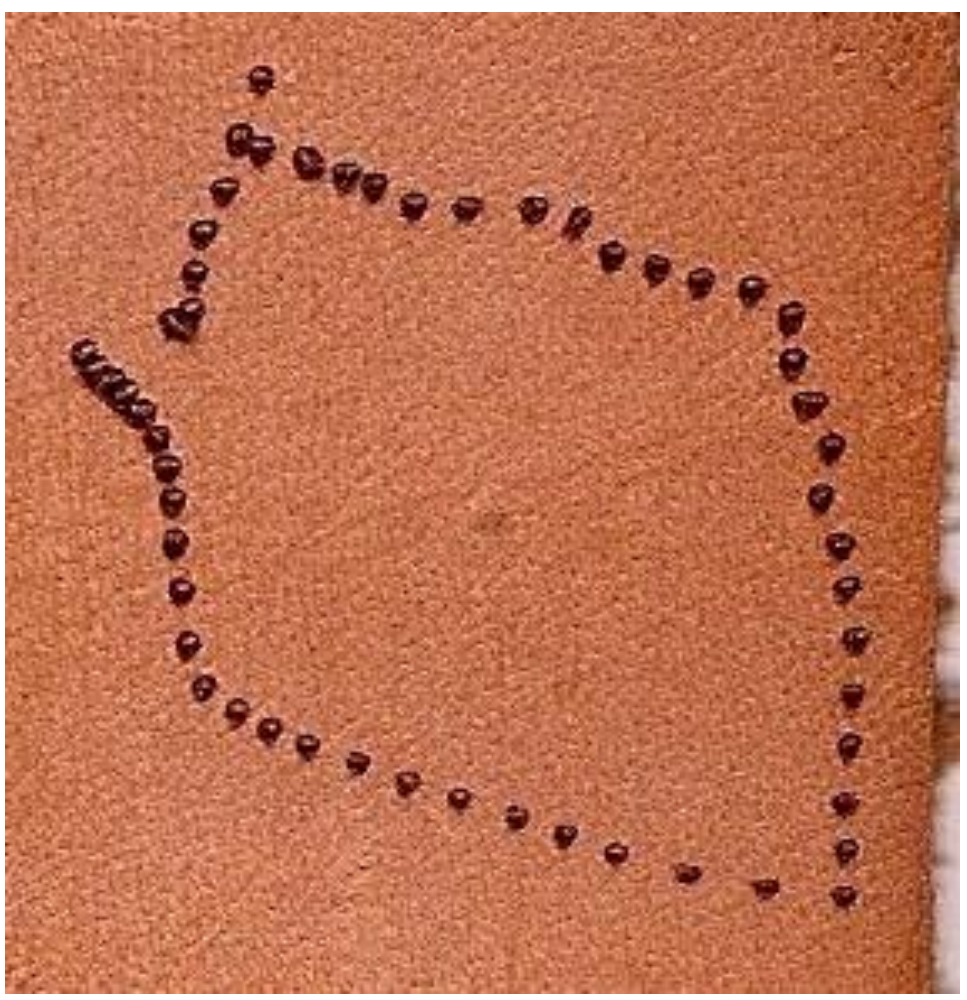

Figure 5.2 Square engraved without Zero method

Square of $14 \mathrm{~mm}$ is engraved. After the first point is engraved, the forces deflect the mirror to second position, which is calculated from the first deflected position of the mirror. This initial distance is kept constant in simulation as well as when the calibration grid is engraved. As the micromirror is deflected, all the magnets are not in the same plane neither at the same constant distance of $3 \mathrm{~mm}$. This results in change in shape and size of the engraved pattern. To overcome this problem, a new method is identified which improves the better control and precision of the micromirror. This method is known as Zero Method, in which the mirror is brought to its initial stage after every single point is engraved. This is explained in the next section.

\subsubsection{Zero Method}

In this method, the main idea is to have a constant distance of initial $3 \mathrm{~mm}$ between magnets and electromagnets when the magnets attached to the mirror experiences the force generated by the actuation of the electromagnet. The process of applying the series of combination of the current is as follows:

1. The first set of the current combination is provided to the electromagnet which causes the mirror to rotate at some angle. 
2. After settling of the micromirror i.e. $370 \mathrm{msec}$, the laser is $\mathrm{ON}$ for $65 \mathrm{msec}$. Increase in the laser $\mathrm{ON}$ time results in the drifting of the polymide substrate due to heat.

3. After $65 \mathrm{msec}$ has passed, the laser is turned OFF and Zero voltage is applied to each electromagnet, which causes the mirror to come back to its initial position.

4. There is again a wait for the next input combination of current, i.e. $400 \mathrm{msec}$, ensuring that the mirror has achieved its initial position and it is at rest.

This entire process is followed by desired points until the pattern is engraved. Below image is of the engraved square of same size. Below is the image of the engraved 14mm square with the Zeromethod applied.

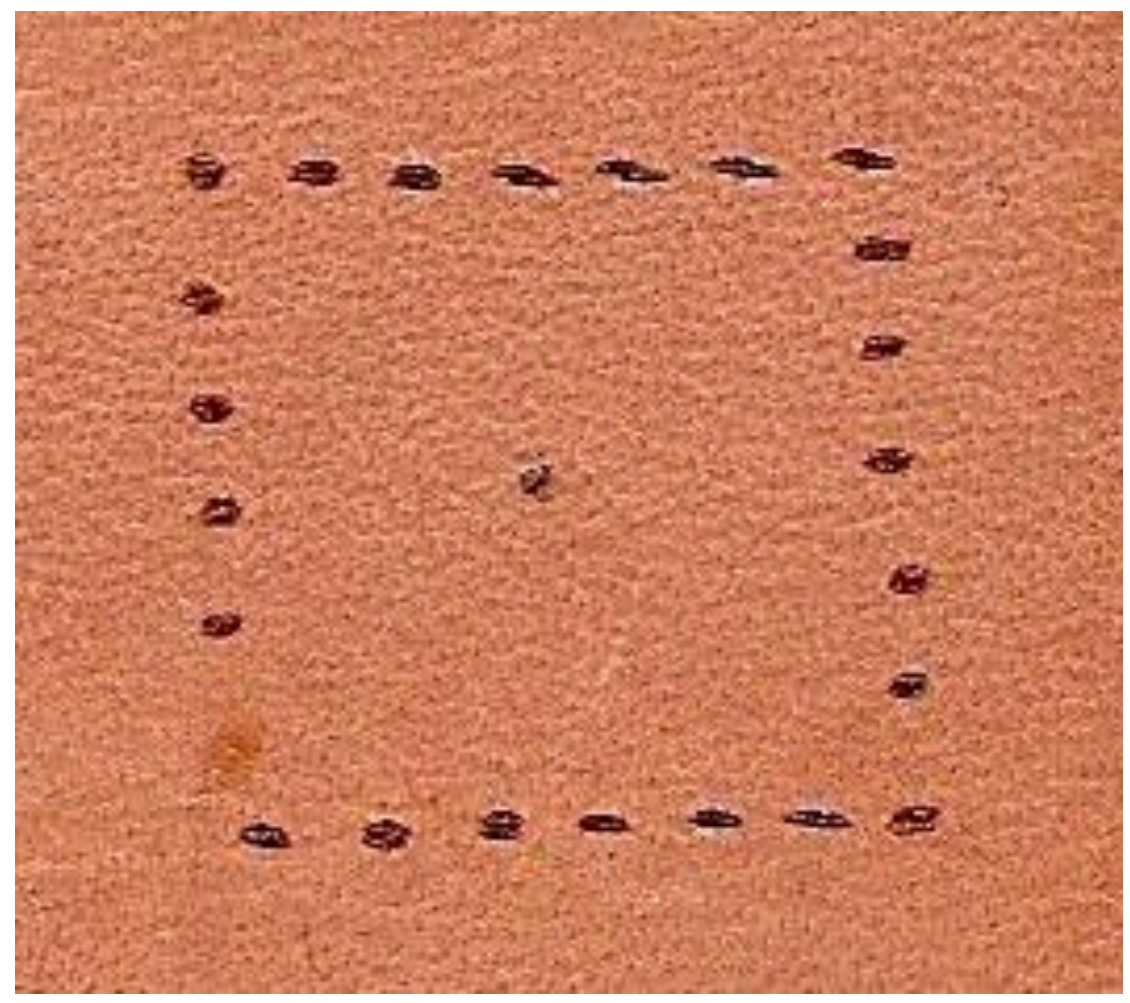

Figure 5.3 Square engraved with Zero method

\subsubsection{Centroid Plot}

Centroid plot is the plot between the Euclidean distance of $\mathrm{X}-\mathrm{Y}$ coordinate from the center versus the angle subtended by that point. It gives a comparable idea of how much the engraved point is moved from its original distance. In order to calculate the plot, the $360^{\circ}$ is divided into equal $9^{\circ}$ span giving us 40 points. In this plot, the second problem which is described afterwards is taken 
care of for better understanding of the facts. Below is the centroid plot comparison. Similarly, this graph can be made for different shape with different comparing parameters. This is based on the above square of $14 \mathrm{~mm}$.

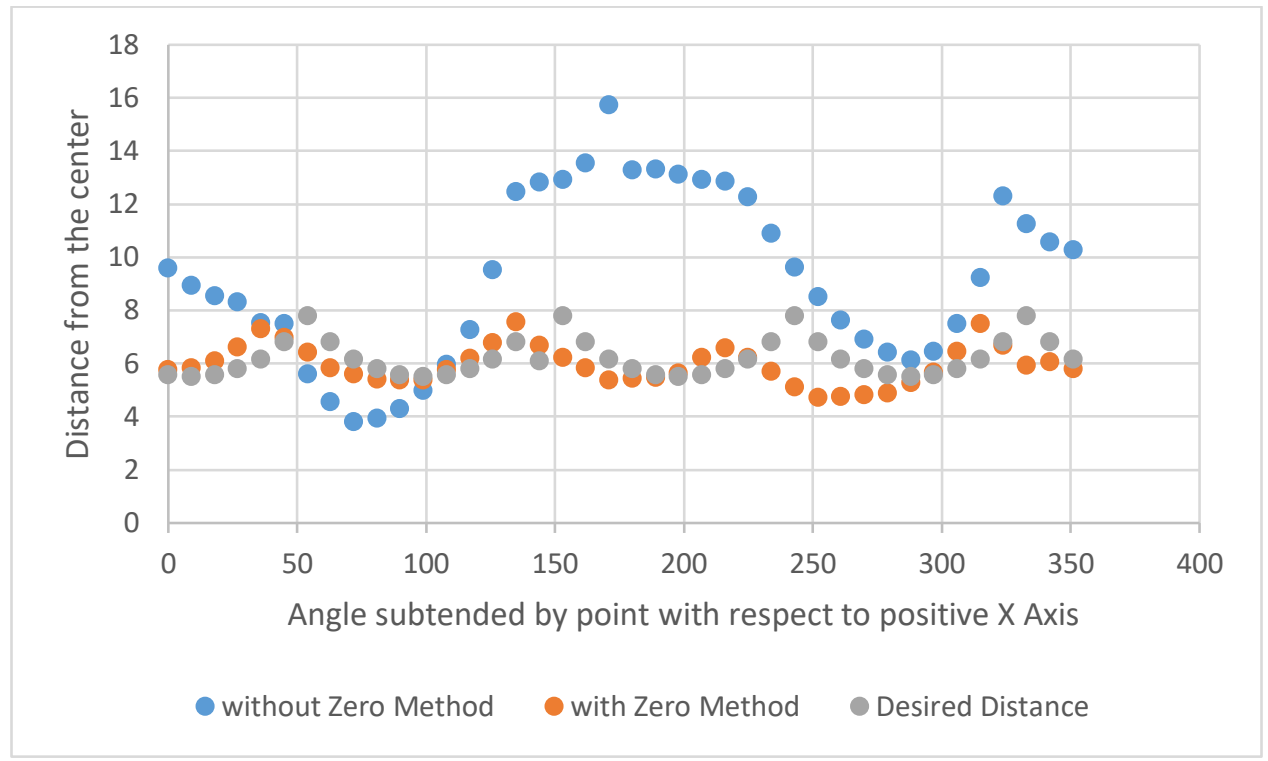

Figure 5.4 Centroid plot between engraved Square by Zero method, without Zero method and exact points

The graph clearly depicts the variation of the engraved image when no zero method is applied and when the zero method is in effect. The average error is $3.64 \%$ between the desired and with the Zero Method.

\subsection{Calibration Problem}

To engrave any desired pattern, the laser beam must be steered to the desired point. The combination of the voltages must be applied to the electromagnet to reach the location and then engraving can be done. As described above, 3-dimensional curve were generated in MATLAB and basic interpolation technique were used. The reason behind this was to calculate the voltage combination of any coordinate within the calibrated space using interpolation of the curves. However, simple interpolation techniques such as B-harmonic spline, Thin plate spline etc. did not provide the precise coordinates of the desired point. 


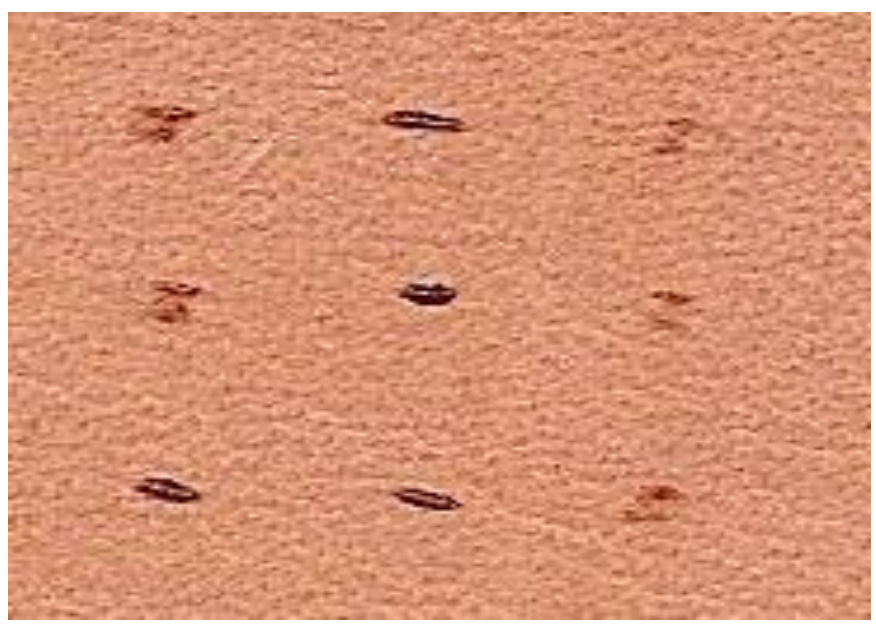

Figure 5.5 Distorted square

The horizontal length of the above engraved leather is around $20 \%$ bigger than the actual desired length. Moreover, the vertical distance is shorter by $18 \%$. This voltage combination supplied to electromagnet doesn't steer the laser beam to the exact desired point given by the user. By using this approach of simple interpolation, there is an evident difference in the shape and size of the engraved and actual pattern.

\subsubsection{Using software Table Curve 3D}

As the entire scanning area is divided into pixel location points and each point is governed by sets of 3 electro-magnetic currents. It is essential to find the method to calculate the current combination of any point desired by the user. Also, as various interpolation technique is tested and there is no significant improvement, non-parametric grid estimation method of finding intermediate values is used. In this method, the space between two points is divided into a small finest grid and only the nearest point values are needed to calculate the current combination for the coordinate. This approach is entirely based on the software used, i.e. Table Curve 3D and there is no provision in the software to find the series of points simultaneously, so it is difficult to calculate the individual points (minimum 100-300 points are needed to make a line contour). 


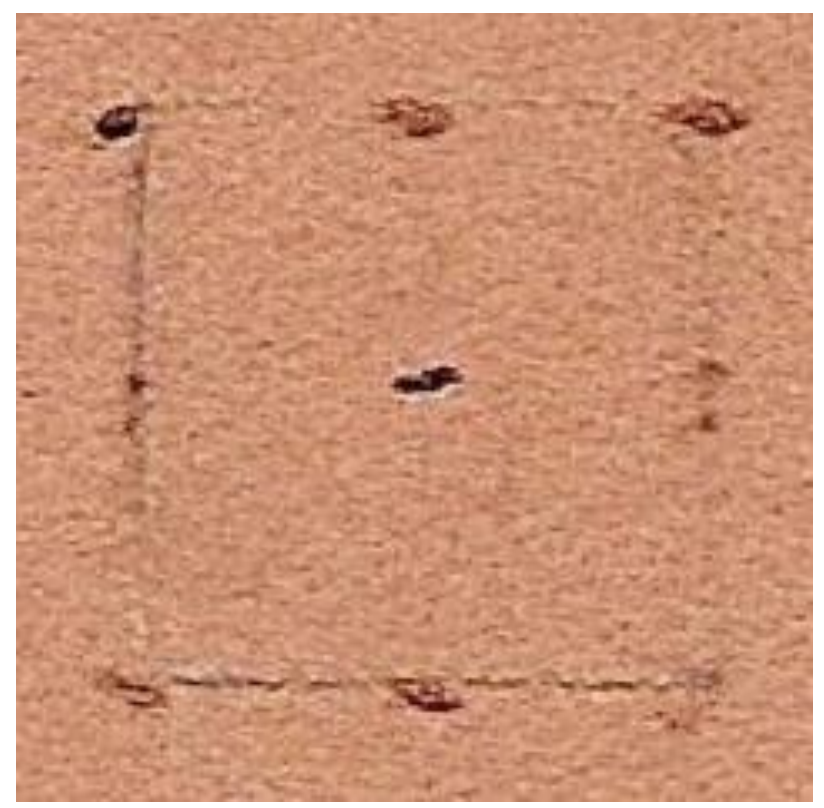

Figure 5.6 Corrected engraved square

More the number of points more accurate would be the engraving. This method reduces the average error percentage to $0.8 \% \sim 1.2 \%$. The error is calculated by finding the distance between the desired and engraved point and then dividing by the distance of the desired point from the origin. However, to calculate a number of points polynomial fit using the same software is done. As per the software fitting statistics, the equation "Chebyshev X, Y Bivariate Polynomial Order 10" fits the scattered point at R-square error value of 0.99967 , which causes the error of $2 \%-3 \%$ error in $\mathrm{X}$ and $\mathrm{Y}$ coordinates which is under the permissible limit.

\subsection{Experimental Verification and Steps for Engraving Pattern}

\subsubsection{Engraving the calibration grid}

To calculate the current combination of the engraved pattern, the micromirrors have to be calibrated. As it is a non-linear mirror, more the number of calibrations more the accuracy of the engraved pattern. To engrave the calibration grid, 441 points are input into the LABVIEW program and the engraving is done on the leather piece. In the starting the laser beam is aligned with the laser source and origin $(0,0)$ is marked on the leather where the laser strikes. This is made that all the coordinates will be measured with reference to this point. Zero method is used to engrave 441 points on the leather. It should be noted that there shouldn't be any change in the micromirror and in the setup, as it might change the configuration and could lead to faulty results. Once all the 
points are engraved, it is visually inspected for any possible drifting or any other defects. Without changing any parameter, once again the code is run and the leather piece is re-engraved on the same spot. By doing this, it makes the point relatively darker and it also eliminates the possibility of the error caused by drifting. Each point takes $835 \mathrm{msec}$ to engrave.

After engraving the calibration grid, it is scanned to its original size into the computer. The scanned image is then converted into a grayscale image. This increases the image quality which makes it easier to distinguish several points. This image is imported into AutoCAD for finding the coordinates of the point. Add-in feature known as "Click2xls"[54] is used in order to calculate the coordinates. This feature directly generates the point coordinate in the Excel sheet automatically when the user clicks the point in the AutoCAD. In this manner all the coordinates of the engraved grid are calculated, hence making the calibration grid

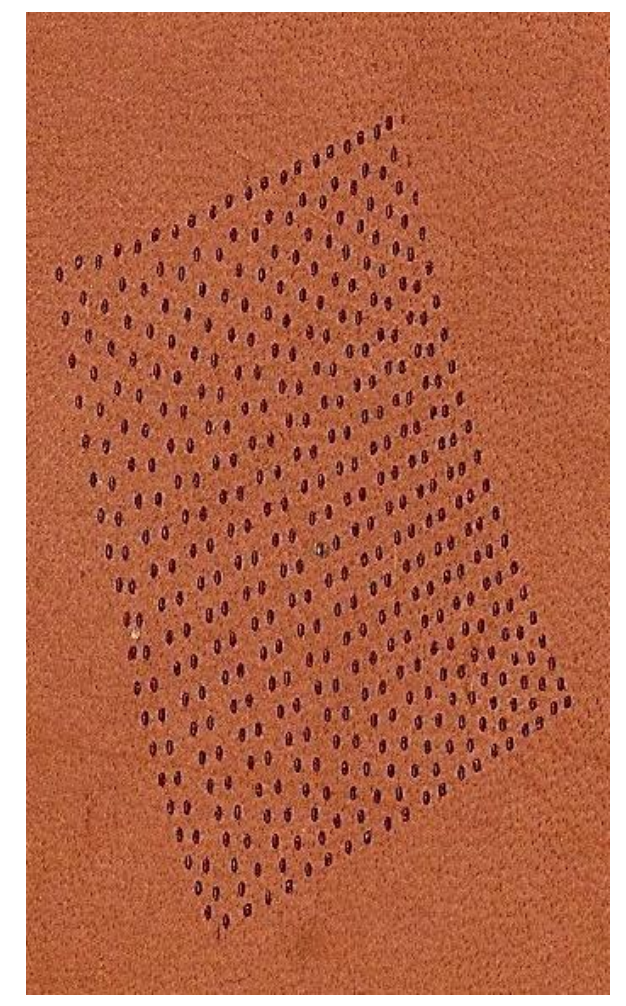

Figure 5.7 Engraved calibration grid

\subsubsection{Plotting the Points into Software Table Curve 3D}

Once the point coordinates and the current combination is generated, now it is important to generate a fitting curve, either based on non-parametric grid estimation or based on the R-square 
valued fit. As the pattern has to be created, number of points are needed which would be difficult to find individually. R-squared value fit is used instead. However, the error would be slightly more in comparison to the other one.

By the software, the graph is generated between 3 parameters at a time. The graph between the coordinates, and each voltage/current is made. After obtaining the three graphs, software is made to run to fit the curve with around 36000 out of 450 million built-in frequently encountered preinstalled set of equations [55]. "Chebyshev X, Y Bivariate Polynomial Order 10” equation fits the curve at about R-square value of 0.99967 . To calculate the voltage corresponding to the pattern to be engraved, from this equation MATLAB code is generated. Three sets of code are generated for the three voltages.

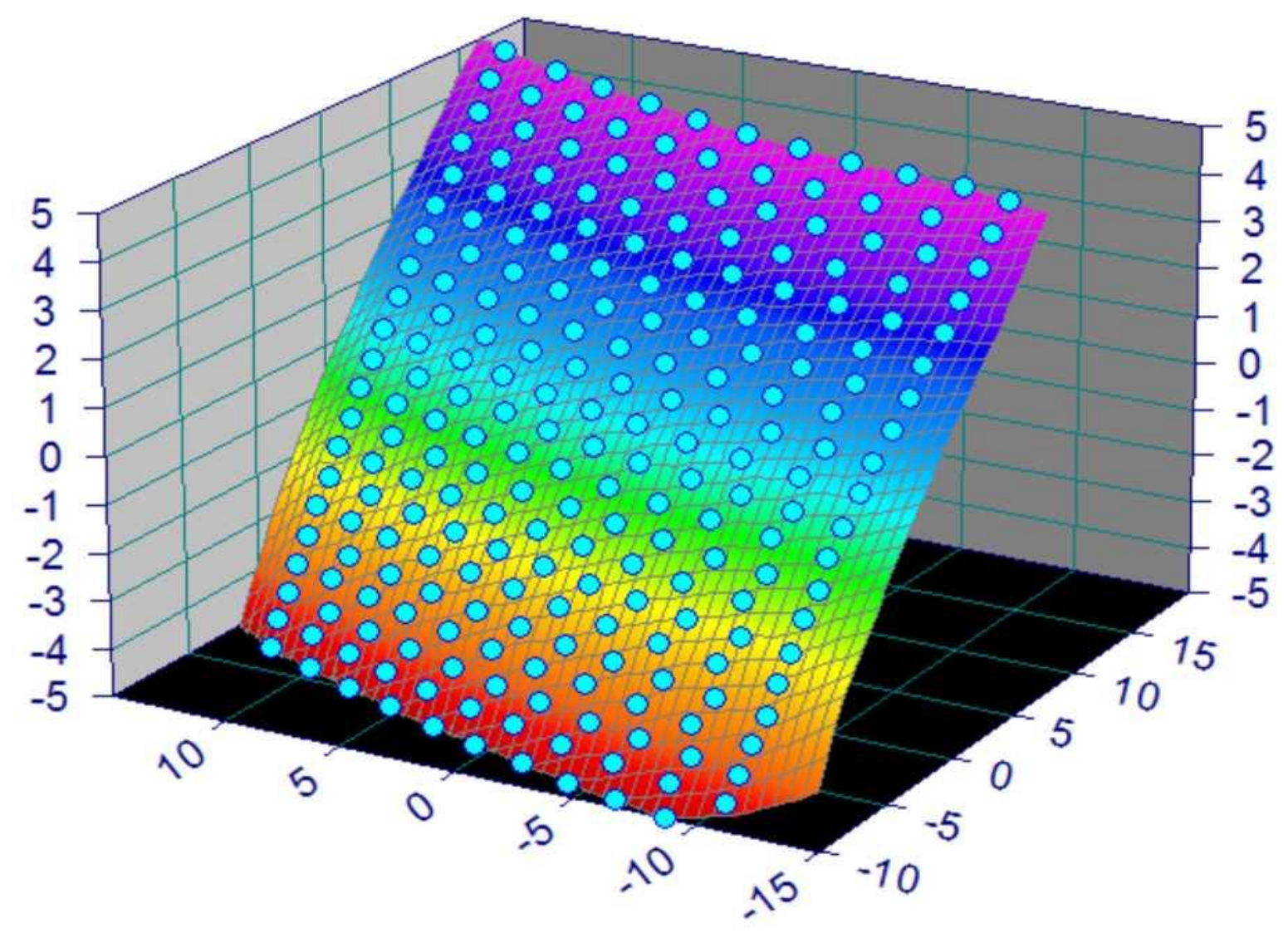

Figure 5.8 Plot between X coordinate, Y coordinate and one of the electromagnet voltages

\subsubsection{Calculating Voltages for Engraving}

Now the coordinates of the pattern to be engraved is to be found so that corresponding voltages can be calculated from the calibrated grid values. To find the coordinates of the pattern, the pattern 
is imported into AutoCAD and the image is divided into a number of the segments. More the number of segments, the pattern would be accurate at the expense of increased engraving time. Once, the segments are made using the "LIST" command of AutoCAD, all the points are retrieved, and these points are then fed into MATLAB to find the corresponding voltage combinations.

Once all the voltage combinations are achieved, the voltage sets are stored individually in column wise text document to be fed into the LABVIEW program. Similarly, the laser ON file values, i.e. depending upon the power needed to be used (3V described earlier) is made in the column fashion. The laser On and OFF is controlled by the LABVIEW program, so all the values would be for the laser on the state. These text files are then being inserted into the program. The very first set is input into the sequence block. The laser is waited for settling time of the mirror to be on i.e. $370 \mathrm{msec}$. After then the laser is on for $65 \mathrm{msec}$. $65 \mathrm{msec}$ is the maximum time after which the FPCB starts to drift due to heat caused by long exposure of the laser beam. This is calculated based on the repeated experiments. Then the 0 values are applied to each electromagnet as well as to the laser. There is then a wait for another $400 \mathrm{msec}$ (more than the settling time of the micromirror), just to ensure that the mirror is once again stable for the next input. Based on this Zero method several leather pieces are engraved. To evaluate the accuracy of the system, small and big squares and circles are engraved.

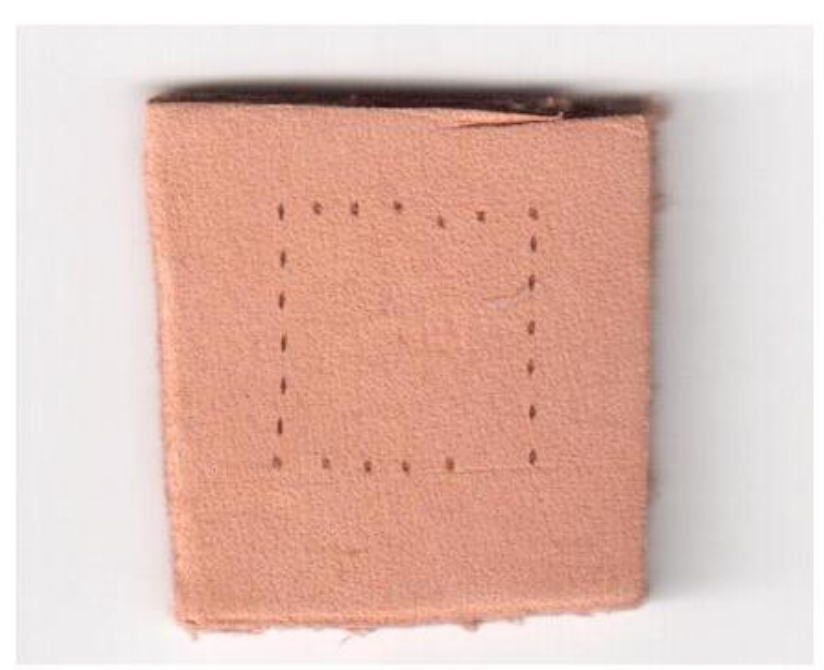

(a)

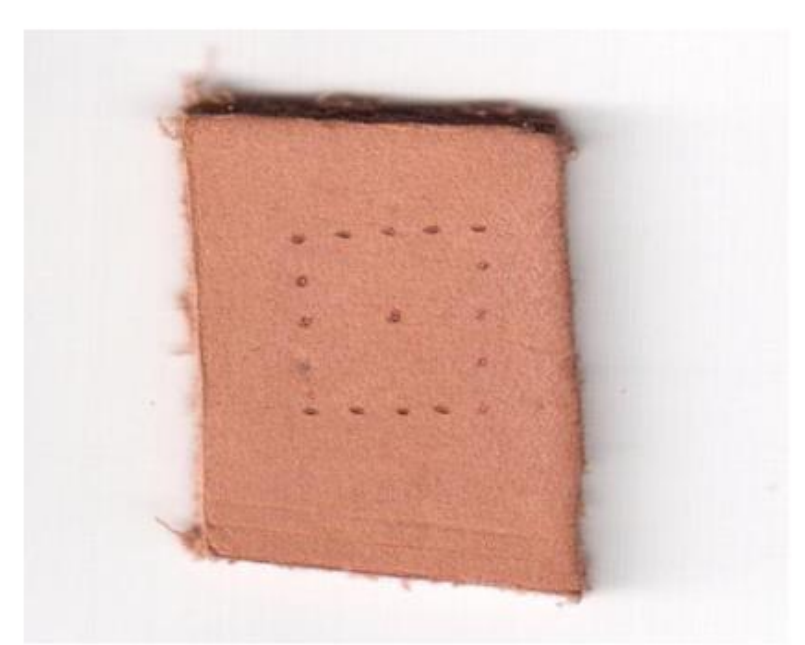

(b)

Figure 5.9 Engraved square (a) $14 \mathrm{~mm}$ (b) $7 \mathrm{~mm}$ 


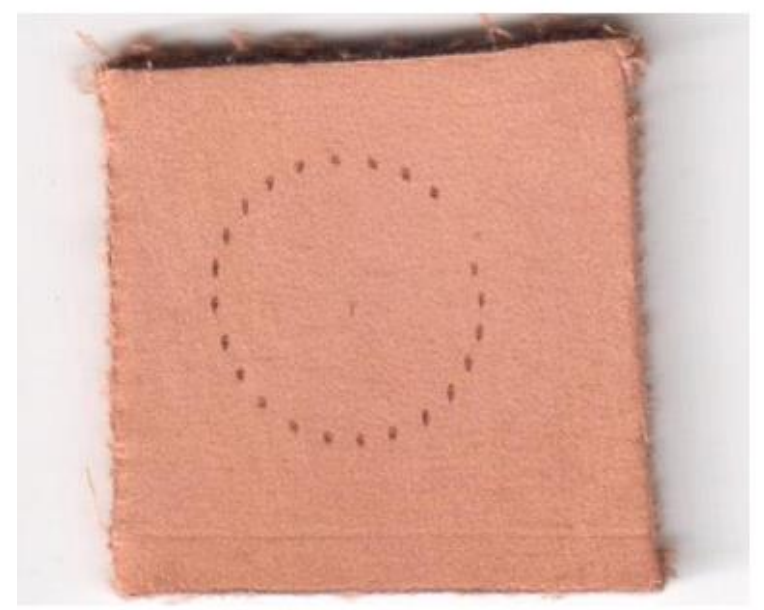

(a)

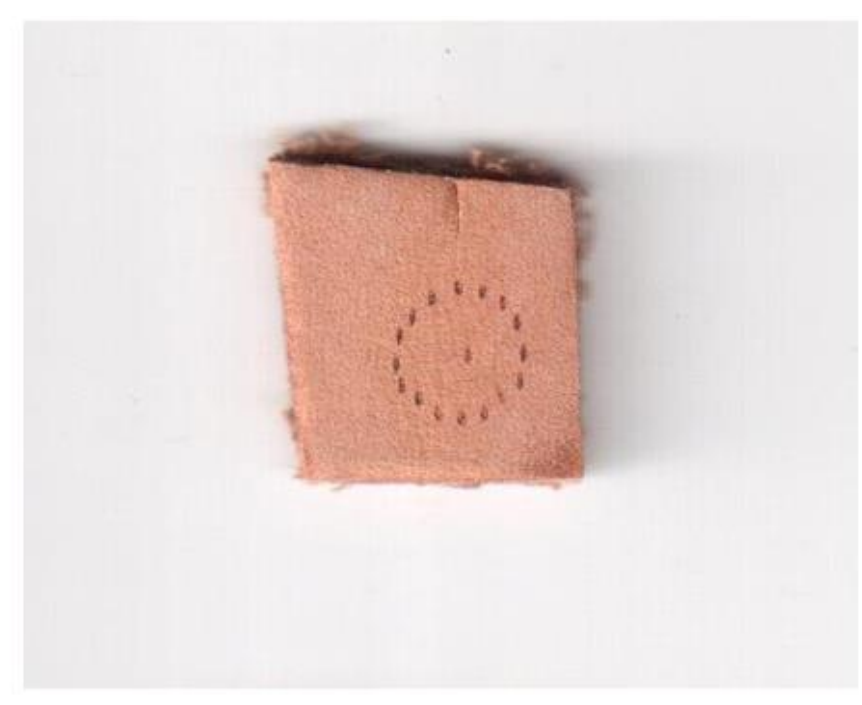

(b)

Figure 5.10 Engraved Circle (a) $14 \mathrm{~mm}$ diameter (b) $7 \mathrm{~mm}$ diameter

Below patterns are engraved using an only a single pass of the laser beam. To compare the engraving on different power values, leather was engraved at different power levels $(970 \mathrm{~mW}$ and $400 \mathrm{~mW}$ ) and then scanned ensuring the accuracy of the leather pattern engraved.

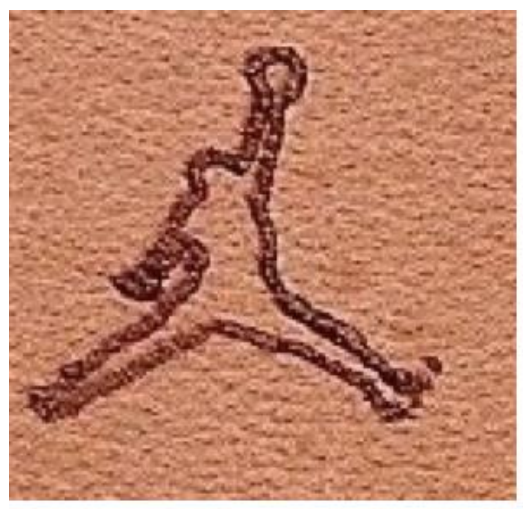

(a)

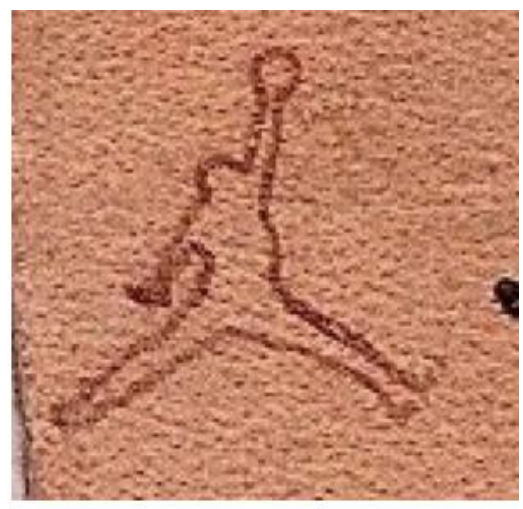

(b)

Figure 5.11 Jordan logo engraved (a) at higher power (b) at lower power 


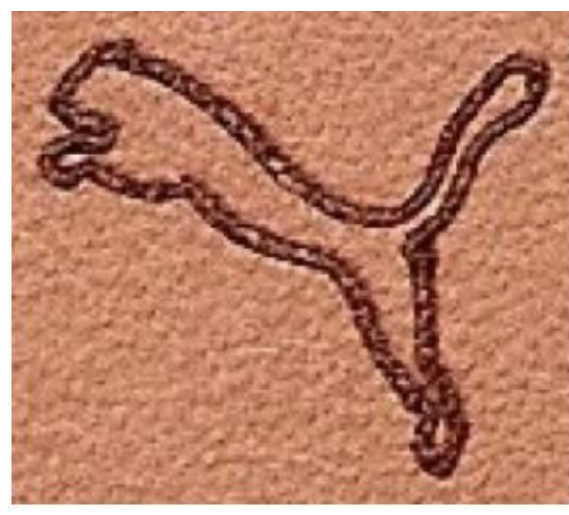

(a)

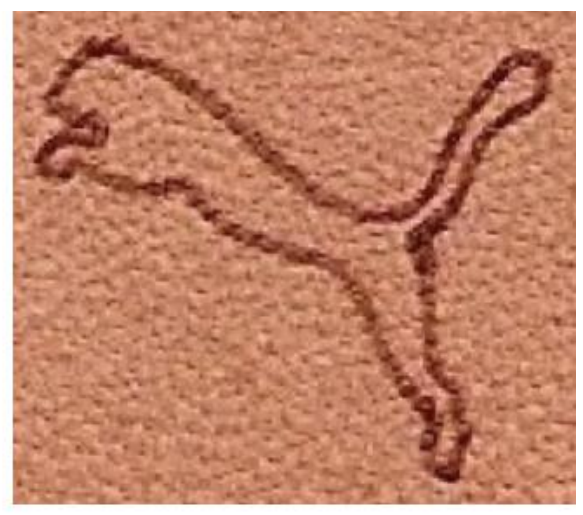

(b)

Figure 5.12 Puma logo engraved (a) at higher power (b) at lower power

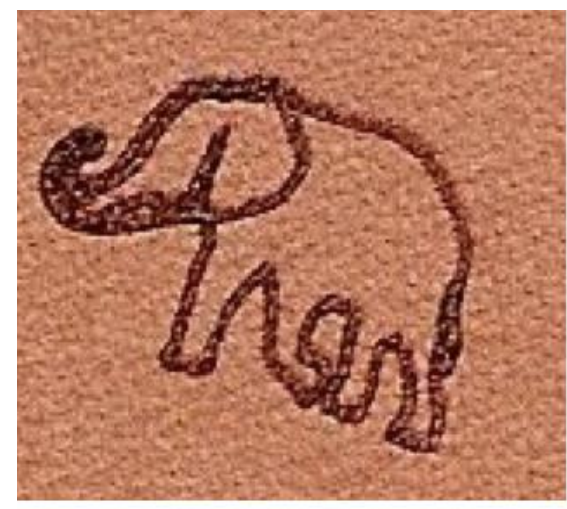

(a)

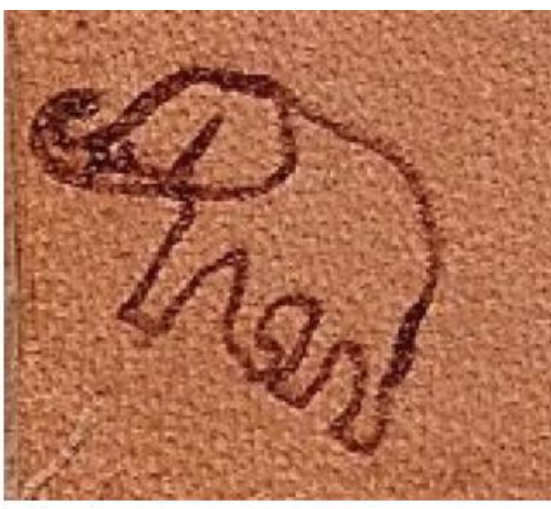

(b)

Figure 5.13 Elephant logo engraved (a) at higher power (b) at lower power

The patterns engraved are at $65 \mathrm{msec}$ laser $\mathrm{ON}$ time. The biggest pattern size that can be engraved is $20 \mathrm{~mm} * 25 \mathrm{~mm}$. For both the elephant pattern, multiple passes were done to engrave on leather. As the data points for the engraved pattern were calibrated on the 10-degree polynomial of the software, the patterns have slight variation from the desired images of $3 \%$ to $4 \%$. However, drifting which is normally associated with the quasi-static FPCB based laser engraver is significantly minimized by using the proposed Zero method and by calibrating the points by the software. The current two- dimensional quasi-static FPCB based micromirror can be used in low power portable Laser marking/ engraving purposes. This method helps in achieving the precise engraving pattern. Below is the image when the leather is engraved with zero method and without zero method. It is observed when the laser is moved directly from one point to another the force in the magnets experience the force generated by the electromagnet at a different distance (not the starting distance 
of $3 \mathrm{~mm}$ ), as the mirror has already been displaced/ rotated to reach to the first point. Now if the next point is input the forces would be calculated based on the changed distance between magnets and electromagnets. This resulted in a significant change in the desired pattern and the engraved one.

Above proposed solutions can be implemented for better control and precision of this laser scanner used in current engraving prototype. With the help of proposed Zero method and using proper calibration for the micromirror, it is evident from the engraving that the problem of drifting caused by a viscous-elastic FPCB micromirror is significantly minimized or eliminated. This makes the current prototype suitable candidate for low cost, compact laser marking system.

\subsection{Chapter Summary}

In this chapter, the calibration technique for the current porotype is discussed. While performing experiments, different problems were encountered. This chapters deals with all the identified problems and solution is proposed to minimize/ mitigate the error caused due to these problems. A new method is proposed which helps in controlling the movement of the micromirror and also increases the accuracy of the result samples. Various logos were engraved at different power using this prototype. All the samples engraved were done 7-8 times in order to check the repeatability of the laser marking/ engraving system. 


\section{Chapter 6 Conclusion}

The entire work can be summarized as follows:

1. The 2-dmensional quasi-static mirror was developed in order to steer the incident laser beam in 2-dimensional space.

2. Modelling and the simulation were carried out to check the viability of the proposed new design.

3. The developed micromirror scanner was successfully used as a scanner in the laser marking/ engraving system in place of two 1-dimensional micromirror scanner. Few noticeable benefits can be achieved:

a. This 2-dimensional micromirror scanner can withstand higher power $(970 \mathrm{Mw})$ than two 1-dimensional micromirror prototype due to single reflection. There is more loss of power in case of two 1-dimensional mirror due to multiple reflection.

b. This prototype is easy to align with the incident laser beam. There is no such case of undershoot and overshoot of laser as in the case of two 1-dimensional mirror scanner.

c. This prototype is smaller than the size of two 1-dimensional micromirror because of only one mirror arrangement. The overall size is $28 \mathrm{~mm} * 28 \mathrm{~mm} * 28 \mathrm{~mm}$.

4. Methods are proposed to overcome the identified problems while performing experiments. The drifting/ creep associated with the FPCB based micromirrors can be minimized by the proposed zero method.

It is evident from the thesis work that the developed low cost quasi-static electromagnetic twodimensional micromirror scanner would be a viable candidate for the replacement of the expensive conventional MEMs bonded mirrors used in the laser marking/ engraving systems. There is a need for low-cost portable laser marking/ engraving system for the consumer market due to the increasing trend of customization of products and various applications which require personalized laser marking systems. This prototype serves the basis of the concept of low-cost portable laser marking/ engraving system, however, there is still a lot of work can be done in order to improve the current prototype. This prototype can be improved in several ways, such as: 
1. By increasing the power of the laser, the current portable consumer-oriented laser marking/engraving system can be made into a high power industry-oriented laser marking/engraving/cutting system. This significant improvement can be achieved by

a. Replacing the aluminium coated mirror with the Brag coated mirror with a reflectivity of $99.99 \%$ on the FPCB based micromirror, the power of the laser can be increased keeping the model intact.

b. Increasing the aperture of the micromirror without increasing the overall dimension of the micromirror.

c. Establishing a feedback control system in order to track the laser beam striking the micromirror.

2. Analyzing the material property of the polymide substrate used in the FPCB structure can further reduce the discrepancy between the simulated and the experimental results.

3. A proper bonding mechanism should be developed for the FPCB structure as well as for the external magnets so that the whole process can be repeatable and mass-produced for the industrial applications.

4. The whole system can further be compacted by replacing the laser source with a smaller size laser module. 


\section{Appendix}

Design Sketches of mechanical support parts sent out for fabrication process:

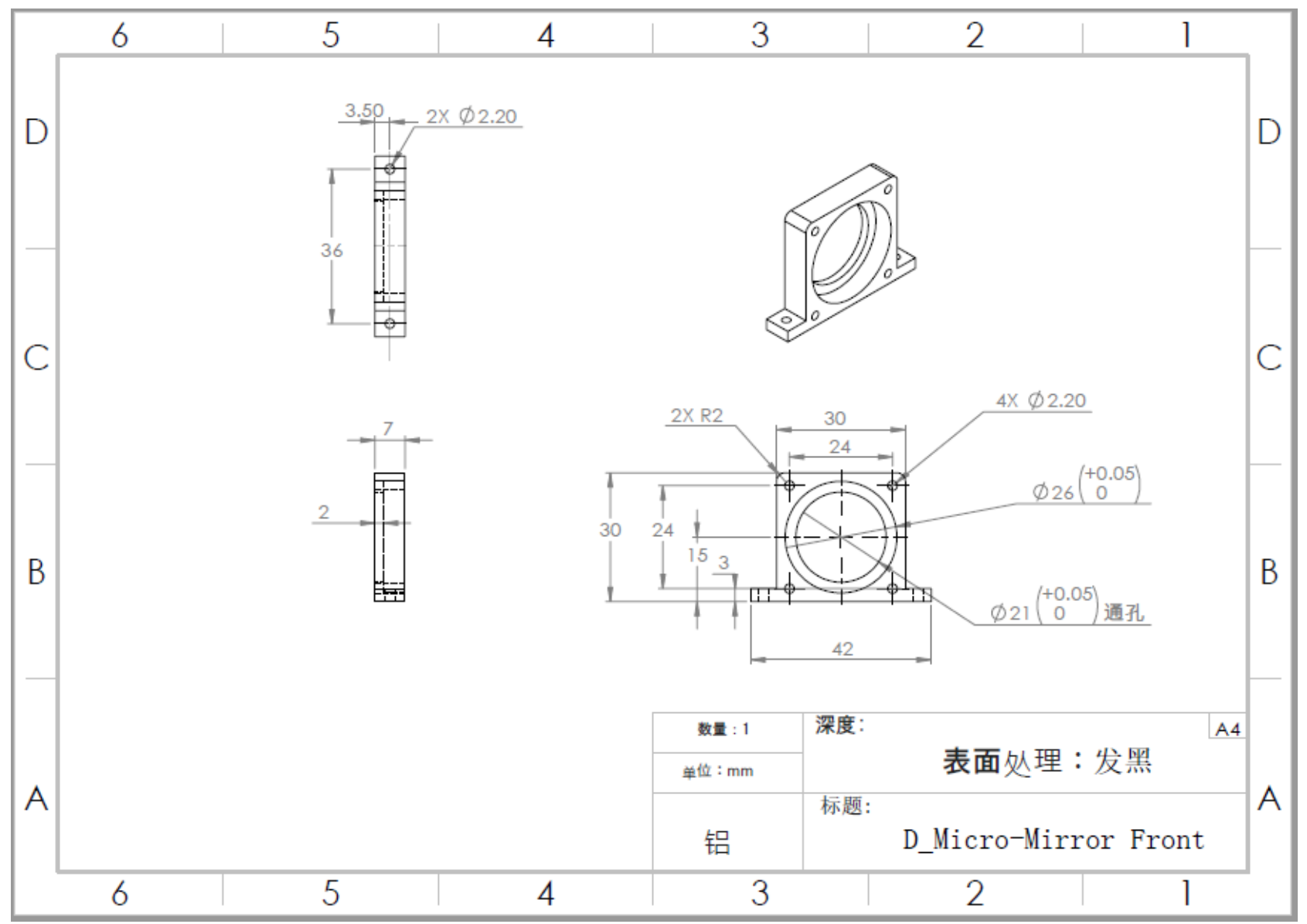



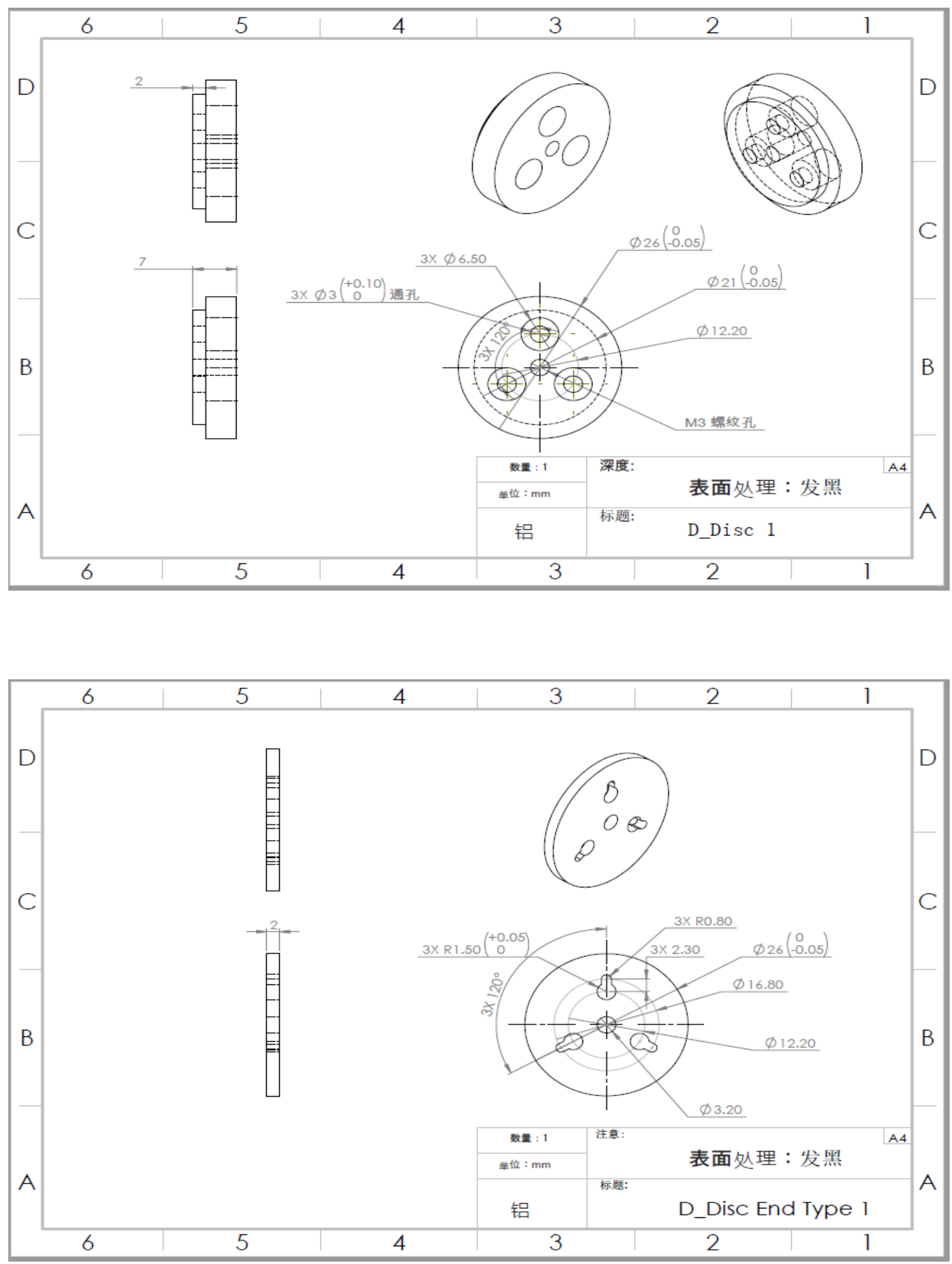

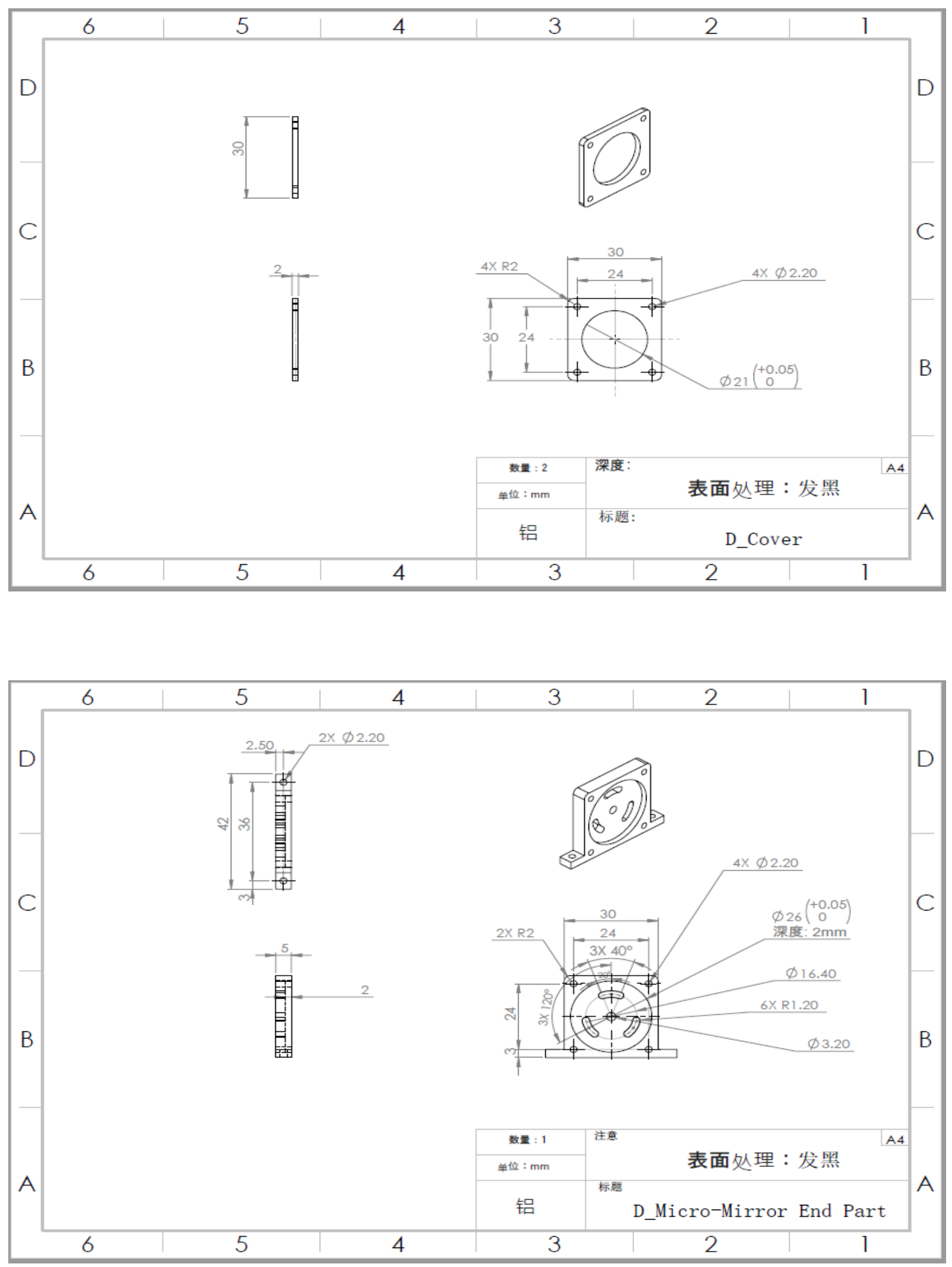

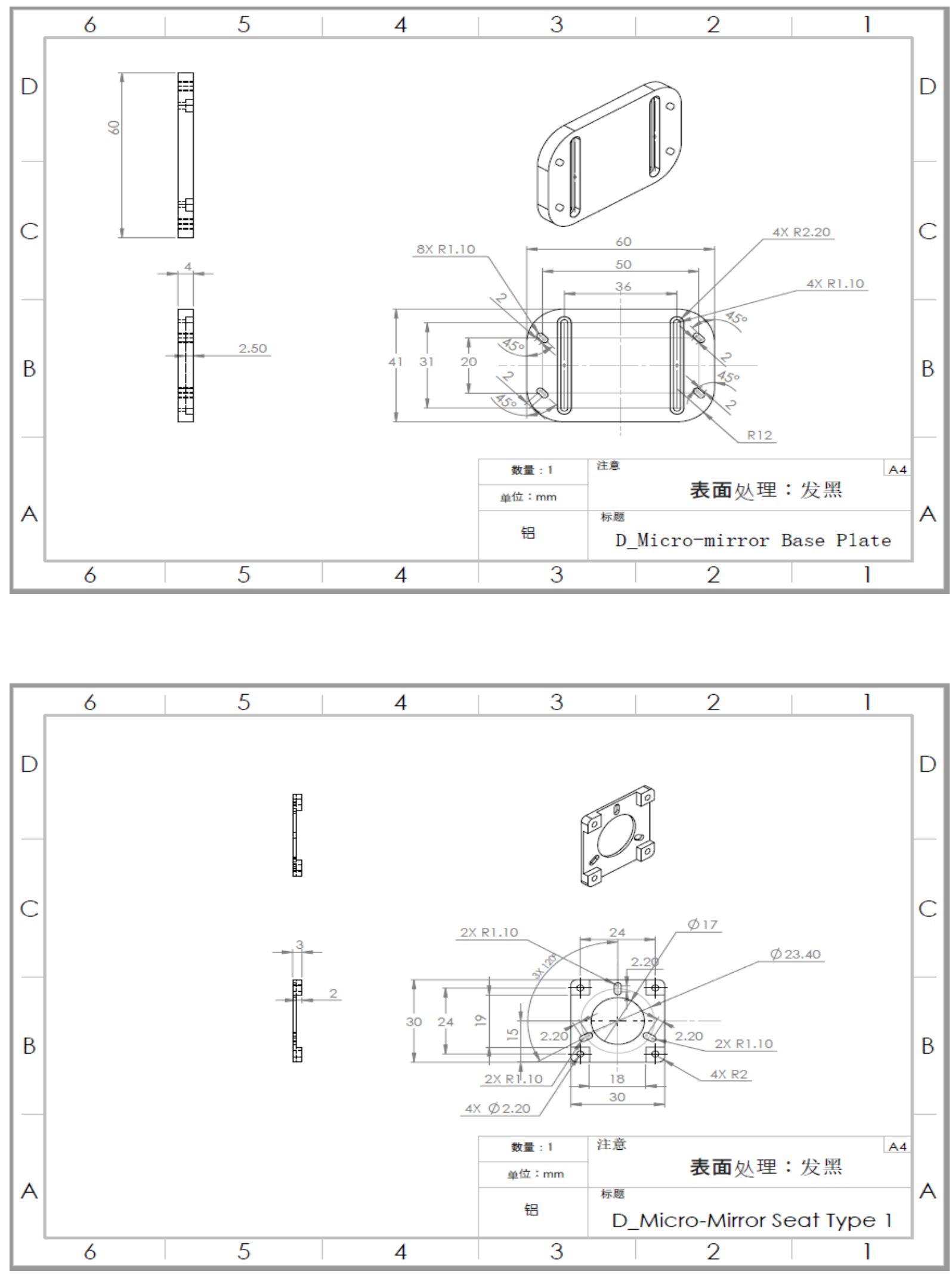


\section{References}

[1] P. J. Ogrodnik, C. I. Moorcroft, and P. Wardle, "The Effects of Laser Marking and Symbol Etching on the Fatigue Life of Medical Devices," J. Med. Eng., vol. 2013, pp. 1-6, Apr. 2013.

[2] What is laser engraving, and how does it work? (n.d.). Retrieved December 15, 2018, from http://digitalskratch.com/how-laser-engraving-works/[Accessed: 15-Dec-2018]

[3] Difference between various laser process (marking and engraving) -service provider website data." [Online]. Available: http://www.lumicision.com/2015/02/difference-betweenlaser-marking-and-engraving/. [Accessed: 15-Dec-2018].

[4] "Comparison of different Laser Types - CO2, Fiber Laser, Vanadat, Yag U-Manufacturer website data." [Online]. Available: https://www.troteclaser.com/en-us/knowledge/faqs/lasertypes/. [Accessed: 16-Dec-2018].

[5] "Laser Modules for Cutting/Engraving (CNC) - manufacturer website data." [Online]. Available: $\quad$ http://odicforce.com/epages/05c54fb6-7778-4d36-adc00098b2af7c4e.sf/en_GB/?ObjectPath=/Shops/05c54fb6-7778-4d36-adc00098b2af7c4e/Categories/Background_and_Projects/Laser_Diode_Cutting_and_Engraving. [Accessed: 16-Dec-2018].

[6] "Wavelength Types used for Laser Marking- manufacturer website data." [Online]. Available: http://www.keyence.com/ss/products/marking/marking_central/select/wavelength.jsp. [Accessed: 17-Dec-2018].

[7] Sat.dundee.ac.uk, W. (. (n.d.). Electromagnetic Spectrum. Retrieved from https://www.sat.dundee.ac.uk/spectrum.html

[8] "Laser machines used for cutting,engraving and marking purposes- manufacturer website data." [Online]. Available: https://www.troteclaser.com/en-ca/laser-machines/. [Accessed: 13Dec-2018].

[9] F. J. C. I. Eur. Ing., Ceng, Laser Processing of Engineering Materials: Principles, Procedure and Industrial Application, 1st ed. Elsevier Butterworth Heinemann, 2005. 
[10] "Laser marking/engraving appliations in various industries-manufactuer website data." [Online]. Available: https://www.troteclaser.com/en-ca/applications/. [Accessed: 12-Dec-2018].

[11] "Epilog Laser Engraving Photo Gallery-manufacturer website data." [Online]. Available: https://www.epiloglaser.ca/how-it-works/anodized.htm. [Accessed: 06-Dec-2018].

[12] "Laser Engraving Training | Industrial Cutting Training -manufacturer website data." [Online]. Available: http://www.vy-tek.com/Help/help-gantry.html. [Accessed: 07-Dec-2018].

[13] G. Ghiani and G. Improta, "The Laser-Plotter Beam Routing Problem," The Journal of the Operational Research Society, vol. 52. Palgrave Macmillan JournalsOperational Research Society, pp. 945-951.

[14] Portable Diode Laser Cutter. (n.d.). Retrieved from https://openbuilds.com/builds/portable-diode-laser-cutter.3414/

[15] "Small Beam Diameter Scanning Galvo Mirror Systems-manufacturer website data." [Online].

Available:

Https://www.thorlabs.com/newgrouppage9.cfm?objectgroup_id=3770\&pn=GVS002. [Accessed: 09-Dec-2018].

[16] Portable Diode Laser Cutter. (n.d.). Retrieved from https://openbuilds.com/builds/portable-diode-laser-cutter.3414/

[17] J. Sun and H. Xie, “MEMS-Based Endoscopic Optical Coherence Tomography,” Int. J. Opt., vol. 2011, pp. 1-12, Jun. 2011.

[18] C.-D. Liao and J.-C. Tsai, “The Evolution of MEMS Displays,” IEEE Trans. Ind. Electron., vol. 56, no. 4, 2009.

[19] J. Chong, S. He, and R. Ben Mrad, "Development of a Vector Display System Based on a Surface-Micromachined Micromirror,” IEEE Trans. Ind. Electron., vol. 59, no. 12, pp. 4863-4870, Dec. 2012.

[20] U. Hofmann and J. Janes, "MEMS Mirror for Low Cost Laser Scanners," in Advanced Microsystems for Automotive Applications 2011, Berlin, Heidelberg: Springer Berlin Heidelberg, 2011, pp. 159-165. 
[21] R. Moss et al., "Low-cost compact MEMS scanning ladar system for robotic applications," 2012, p. 837903.

[22] P.-A. Blanche, L. LaComb, Y. Wang, and M. Wu, "Diffraction-Based Optical Switching with MEMS,” Appl. Sci., vol. 7, no. 4, p. 411, Apr. 2017.

[23] Mirrorcle Technologies MEMS Mirrors - Device Prices for US Sales, Mirrorcle Technologies, Inc., https://mirrorcletech.com/pdf/Mirrorcle Tech_Device_Prices.pdf [Accessed: Dec. 02, 2018]

[24] TALP1000B Dual-Axis Analog MEMS Pointing Mirror, Texas Instruments, 2009. http://www.ti.com/lit/ml/slbb089/ lbb089.pdf [Accessed: Dec. 02, 2018]

[25] Greenspun J T, Massey T L and Pister K S J, “The Mems Hammer, A Tool To Study Microfracture," Hilton Head 2016, Solid State Sensors, Actuators and Microsystems Workshop, June 2016, pp.1-5.

[26] Yi T and Kim C-J, "Measurement of mechanical properties for MEMS materials," Meas. Sci. Technol. 19998, Vol. 10, pp. 706-716.

[27] "Nexgen Portable Laser Marker- Laser Marking/Engraver-Manufacturer website data." [Online]. Available: http://www.nexgenlasermarker.com/about. [Accessed: 13-Nov-2018].

[28] "MEMS mirror price list- manufacturer website data." [Online]. Available: http://mirrorcletech.com/pdf/MirrorcleTech_Device_Prices.pdf. [Accessed: 12-Nov-2018].

[29] "NLL 10 label laser by NanoSec Technology GmbH - Product data sheet." [Online]. Available: http://www.nanosec.eu/download/NLL_10_datasheet.pdf. [Accessed: 13-Aug-2017].

[30] V. Milanovic, "Linearized Gimbal-Less Two-Axis MEMS Mirrors," in Optical Fiber Communication Conference and National Fiber Optic Engineers Conference, 2009, p. JThA19.

[31] "Gimbal-less Two Axis (Tip-Tilt) MEMS Mirrors by Mirrorcle Technologies INC manufacturer website data." [Online]. Available: http://mirrorcletech.com/devices.html. [Accessed: 13-Nov-2018].

[32] Karlmarx G. K. Periyasamy, “'FPCB MAGNETIC MICROMIRROR FOR LASER MARKING/ENGRAVING SYSTE,” Master Thesis, Ryerson University, 2017. 
[33] W. Liao, W. Liu, Y. Zhu, Y. Tang, B. Wang, and H. Xie, “A Tip-Tilt-Piston Micromirror With Symmetrical Lateral-Shift-Free Piezoelectric Actuators,” IEEE Sens. J., vol. 13, no. 8, pp. 2873-2881, Aug. 2013.

[34] J. Sam, J. Kumar, E. A. Tetteh, and E. P. Braineard, "A Study Of Why Electrostatic Actuation Is Preferred And A Simulation Of An Electrostatically Actuated Cantilever Beam For Mems Applications,” Int. J. Eng. Sci. Emerg. Technol., vol. 6, no. 5, pp. 441-446, 2014.

[35] Koh, K. H., Kobayashi, T., Hsiao, F., \& Lee, C. (2010). Characterization of piezoelectric PZT beam actuators for driving 2D scanning micromirrors. Sensors and Actuators A: Physical, 162(2), 336-347. doi:10.1016/j.sna.2010.04.021

[36] J. Sam, J. Kumar, E. A. Tetteh, and E. P. Braineard, "A Study Of Why Electrostatic Actuation Is Preferred And A Simulation Of An Electrostatically Actuated Cantilever Beam For Mems Applications,” Int. J. Eng. Sci. Emerg. Technol., vol. 6, no. 5, pp. 441-446, 2014.

[37] C. Livermore, "Design choices: MEMS actuators Motivation." [Online]. Available: https://ocw.mit.edu/courses/electrical-engineering-and-computer-science/6-777j-design-andfabrication-of-microelectromechanical-devices-spring-2007/lecture-notes/07lecture21.pdf. [Accessed: 23-Oct-2018].

[38] Fu, Y. Q., Hu, M., Du, H., Luo, J., Flewitt, A. J., \& Milne, W. I. (2005). Micromirror structure based on TiNi shape memory thin films. Smart Structures, Devices, and Systems II. doi:10.1117/12.567317

[39] Piyawattanametha, W., Patterson, P., Hah, D., Toshiyoshi, H., \& Wu, M. (n.d.). A 2D scanner by surface and bulk micromachined vertical comb actuators. 2003 IEEE/LEOS International Conference on Optical MEMS (Cat. No.03EX682). doi:10.1109/omems.2003.1233483

[40] Y. Xue and Siyuan He, "A translation micromirror with large quasi-static displacement and high surface quality,” J. Micromechanics Microengineering, vol. 27, no. 1, Jan. 2017.

[41] "Laser beam divergence and spot size (Theory) : Laser Optics Virtual Lab: Physical Sciences: Amrita Vishwa Vidyapeetham University Virtual Lab." [Online]. Available: http://vlab.amrita.edu/?sub=1\&brch=189\&sim=342\&cnt=1. [Accessed: 18 -Dec-2018]. 
[42] "F-Theta Scan Lenses database (Thor Labs) -manufacturer website data." [Online]. Available: https://www.thorlabs.com/newgrouppage9.cfm?objectgroup_id=6430. [Accessed: 19Nov-2018].

[43] "F-Theta Scanning Lenses database (Edmund Optics)-manufacturer website data." [Online]. Available: https://www.edmundoptics.com/optics/laser-optics/laser-opticalassemblies/F-Theta-Scanning-Lenses/. [Accessed: 18-Aug-2017].

[44] "3 element / G2 / G7 / Laser focus Lenses (Sanwu Lasers)-manufacturer website data." [Online]. Available: https://www.sanwulasers.org/product/lenses. [Accessed: 20-Nov-2018].

[45] "Nanofabrication: Principles, Capabilities and Limits - Zheng Cui section (3.3.3)," .

[46] S. Franssila, Introduction to microfabrication (section 8). Wiley, 2013.

[47] “DuPont TM Kapton $®$ HPP-ST POLYIMIDE FILM - material datasheet.”

[48] "Hot Rolled and Annealed (O25) C10400 Copper (makeitform.com)-material property database." [Online]. Available: http://www.makeitfrom.com/material-properties/Hot-Rolled-andAnnealed-O25-C10400-Copper. [Accessed: 21-Dec-2018].

[49] "Reflectance - Wikipedia webpage." [Online]. Available: https://en.wikipedia.org/wiki/Reflectance. [Accessed: 20-Dec-2018].

[50] "Laser source (Opt Lasers)- manufacturer website data." [Online]. Available: https://optlasers.com/en/. [Accessed: 20-Nov-2018].

[51] "Learn LabVIEW ( National Instruments)- Software tutorial." [Online]. Available: http://www.ni.com/academic/students/learn-labview/. [Accessed: 22-Aug-2018].

[52] S. Zhang, S. Mori, M. Sakane, T. Nagasawa, and K. Kobayashi, "Tensile Properties and Viscoelastic Model of a Polyimide Film*,” J. Solid Mech. Mater. Eng., vol. 6, no. 6, 2012.

[53] L. Arruda, R. Bonadiman, C. Josineto, and G. Freitas, "Experimental and numerical analyses of flexible PCBs under various loading conditions," in EuroSimE 2009 - 10th International Conference on Thermal, Mechanical and Multi-Physics Simulation and Experiments in Microelectronics and Microsystems, 2009, pp. 1-10.

[54] "Autocad application- \&quot; click2xls\&quot; (CAD Forum)." 
[55] TableCurve 3D. (2018, September 19). Retrieved from https://en.wikipedia.org/wiki/TableCurve_3D 